\title{
Examining the Career Pathways for Women Administrators at a Land-Grant University
}

\author{
Meridith A. Balas \\ West Virginia University, meridith.balas@mail.wvu.edu
}

Follow this and additional works at: https://researchrepository.wvu.edu/etd

Part of the Educational Leadership Commons, Higher Education Commons, and the Women's Studies Commons

\section{Recommended Citation}

Balas, Meridith A., "Examining the Career Pathways for Women Administrators at a Land-Grant University" (2020). Graduate Theses, Dissertations, and Problem Reports. 7942.

https://researchrepository.wvu.edu/etd/7942

This Dissertation is protected by copyright and/or related rights. It has been brought to you by the The Research Repository @ WVU with permission from the rights-holder(s). You are free to use this Dissertation in any way that is permitted by the copyright and related rights legislation that applies to your use. For other uses you must obtain permission from the rights-holder(s) directly, unless additional rights are indicated by a Creative Commons license in the record and/ or on the work itself. This Dissertation has been accepted for inclusion in WVU Graduate Theses, Dissertations, and Problem Reports collection by an authorized administrator of The Research Repository @ WVU.

For more information, please contact researchrepository@mail.wvu.edu. 
Examining the Career Pathways for Women Administrators at a Land-Grant University

Meridith A. Balas

Dissertation submitted to the College of Education and Human Services

at West Virginia University

in partial fulfillment of the requirements for the degree of

Doctor of Philosophy in Higher Education

Erin McHenry-Sorber, Ph.D., Chair

Rodney Hughes, Ph.D.

Kathleen Provinzano, Ph.D.

Nathan Sorber, Ph.D.

Department of Curriculum and Instruction/Literacy Studies

Morgantown, West Virginia

2020

Keywords: higher education, higher education administration, women's leadership,

feminist theory, career pipeline, women in the workplace, patriarchal culture, mentorship, land-grant universities

Copyright 2020 Meridith Balas 


\section{ABSTRACT \\ Examining the Career Pathways for Women Administrators at a Land-Grant University}

\section{Meridith A. Balas}

This feminist-centered, narrative study focuses on the troubling gender gaps in higher education leadership by exploring the career paths and lived experiences of current women administrators at a large, public land-grant institution. This research identifies specific supports and barriers women face throughout their careers that might enable or prevent them from attaining or accessing high-level leadership roles in academic and non-academic administration. The study leans on feminist theory to position perceptions of women in leadership roles across many disciplines in the university setting while observing it as a critical lens to analyze gender inequality in the career pipeline for women leaders in higher education.

The research reveals the pervasiveness of ongoing gender-based discrimination that is embedded into the culture of many universities and faced by women faculty, staff, and administrators every day. The study contains practical implications for large, public land-grant and similar institutions, specifically related to a growing need for gender-diverse leadership at every level of university administration, the effectiveness of focused in-house women's leadership programs, and the ongoing positive impact of intentional supervision and prioritized mentorship. This work contributes to the literature its examination of women's experiences in both academic and non-academic leadership positions in the same institutional context. 


\section{Table of Contents}

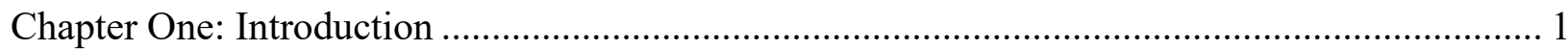

Statement of the Problem ................................................................................................. 5

Purpose Statement and Research Questions ........................................................................ 6

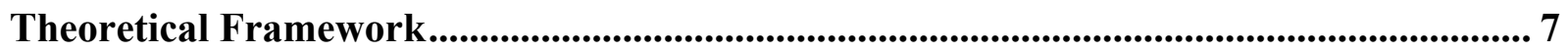

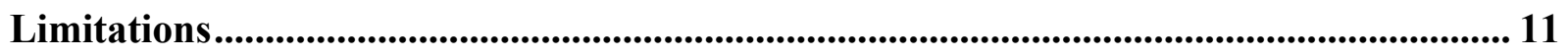

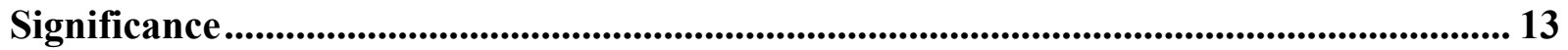

Definition of Key Terms .............................................................................................................. 14

Conclusion

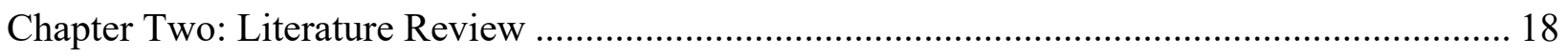

Key Concepts ....................................................................................................................................... 21

Challenges for Women Administrators in Higher Education .............................................. 27

Organizational Impact................................................................................................................... 48

Gender and Leadership at Land-Grant Institutions ........................................................... 50

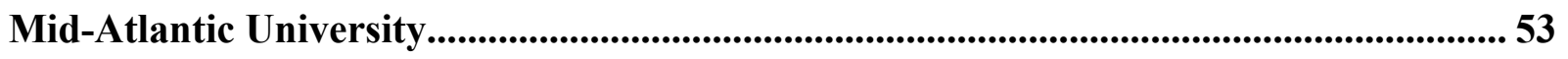

Women's Initiatives at Mid-Atlantic University .................................................... 55

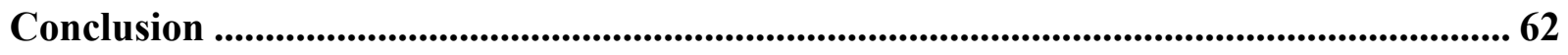

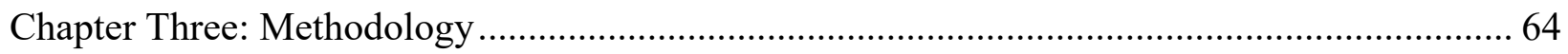

Overview of Methodology .....................................................................................................6 65

Conceptual Framework.................................................................................................................. 69

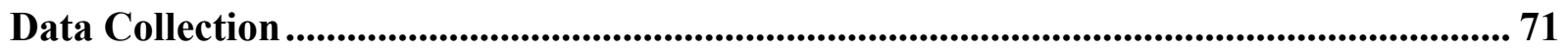

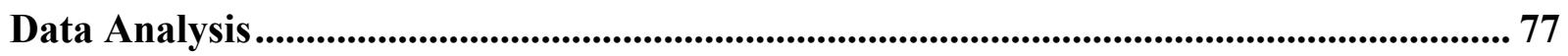

Trustworthiness of Research .................................................................................... 80

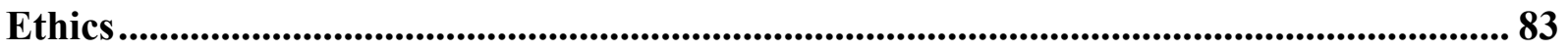

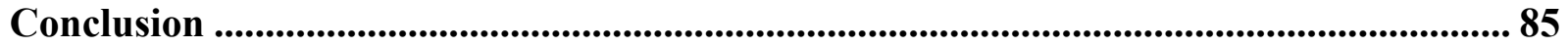

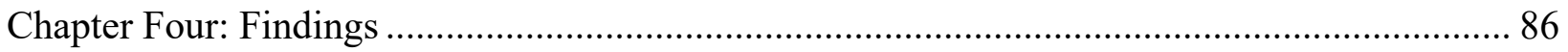

Participant Overview ............................................................................................. 87

Theme 1 - Attrition Points and Positive Influences......................................................... 90

Theme 2 - Pervasiveness of Gender-Based Discrimination ......................................... 113

Theme 3 - Further Evidence of a Service Burden ................................................................ 133

Theme 4 - Strategies for Navigation and Survival ........................................................... 138

Theme 5 - Progress at Mid-Atlantic University ............................................................. 144 
CAREER PATHWAYS FOR WOMEN ADMINISTRATORS iv

Additional Findings: Reflection and Meaning Making .................................................... 148

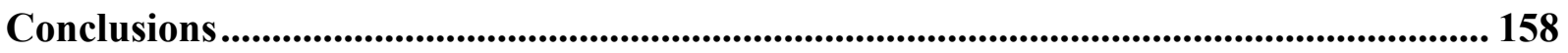

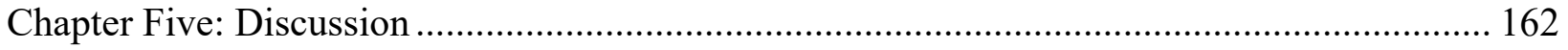

Summary of Findings .................................................................................................................. 164

Implications for Practice ................................................................................................. 169

Limitations and Context................................................................................................. 184

Directions for Future Research ................................................................................... 190

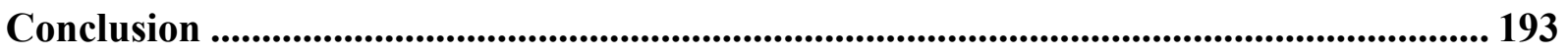

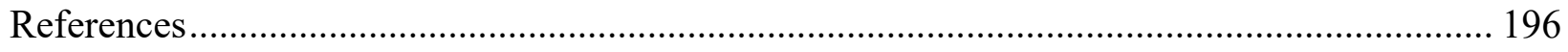

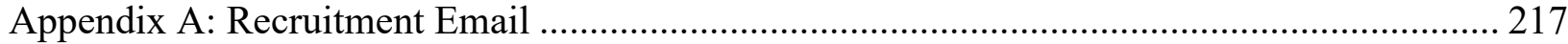

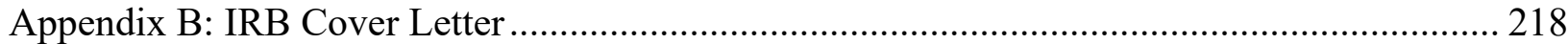

Appendix C: Interview Guide ......................................................................................... 219 
I raise up my voice - not so that I can shout, but so that those without a voice can be heard...

To ensure freedom and equality for women so that they can flourish. We cannot all succeed when half of us are held back.

-Malala Yousafzai

Address to the United Nations Youth Assembly, 2013 


\section{Dedication}

In loving memory of James Gismondi.

For kindly providing me with my first educational scholarship, for modeling the importance of giving back to your alma mater and community, and for spotlighting education as a tool to create a brighter future for all - I am so very grateful. 


\section{Acknowledgments}

To the women who so bravely shared their time and stories for this study in hopes of clearing an easier path for the women who will come after them, I thank you from the bottom of my heart.

To my advisor, Dr. Erin McHenry-Sorber, whose approach of empathy and care led me through this process from start to finish - I am incredibly grateful for the supported space you have cultivated for me to learn and grow in as a student, as a researcher, and as an instructor.

To my committee, Dr. Sorber, Dr. Hughes, and Dr. Provinzano, whose wisdom and expertise focused my research and prepared me to be a more thoughtful scholar, I am endlessly appreciative.

To my steadfast circle of friends and family, I am humbled and motivated every day by your unwavering confidence in me.

To my loyal partner, for always being willing to debate higher education politics over coffee, for waiting patiently for the "after I defend my dissertation..." moments, and for believing in my deepest motivations and aspirations; I am so thankful.

To the kind and assiduous instructors at Lafayette Elementary, Chestnut Ridge Christian Academy, Benjamin Franklin, Uniontown High School, Penn State University, the University of Georgia, and West Virginia University: thank you for emboldening my curiosity and for creating environments where I could fall in love with education over and over again.

To the CSAA-M faculty at the University of Georgia, thank you for offering the perfect balance of challenge and support, for equipping me with an appreciation for APA style, and for awakening my interest in feminist research.

To Sheldon Calvary Camp, for being the most influential classroom I've ever had the opportunity to learn in, and for instilling in me the sustaining values of inclusion, responsibility, empathy, and individualization. 
Chapter One: Introduction 
Ongoing national research reveals that academic career pathways remain stratified by gender, race, and class, a phenomenon that contradicts the historical position of higher education attainment as a means to increase power, status, and success (De Welde \& Stepnick, 2015). As some of the most historical and innovative universities today, land-grant institutions are often looked to as examples; yet many of these land-grant colleges and universities rely on outdated systems of gendered leadership environments while aiming to advance. It is clear that there are significant barriers preventing women leaders from advancing to high-level administrative roles and with a more diverse corps of leadership that features men and women equally valued, supported, and represented, these institutions could benefit from divergent perspectives and unique lenses that affect the lives of students, faculty, and staff.

The status of women within the context of higher education today is a result of historical circumstances and the confluence of social and political forces (Allan, 2011). Since the early 1800s, women's roles in higher education have shifted with each historical movement, starting with women's desire to obtain an education in the 1830s and evolving as wars and progression identified new professional roles for educated women (Parker, 2015). However, it was not until the 1960s and 1970s that equality in the workplace became an integral part of the national conversation, leading many organizations to become focused on job responsibilities and progress instead of gender (Parker, 2015; Tuttle, 2004). Though strides have been made for women in pursuit of high-level leadership positions, the highest offices still represent strongholds for masculinity; until women are equally represented in all facets of society and until leaders prioritize and challenge issues to combat inequality, women will continue to be a target of discrimination in the workplace (Jalalzai, 2008).

Women are outnumbered and underrepresented in academic leadership positions, in absolute numbers, and in proportion to the eligible pool of women (Dominici et al., 2009). In 
2011, the men to women ratio of public universities in the United States was 43.6-56.4 (Borzelleca, 2012). With women only holding $27 \%$ of presidencies, postsecondary institutions are led by a majority of men, a figure which deviates greatly from the ratio of women to men on college campuses today (Borzelleca, 2012; Johnson, 2016). Women have earned more than 50\% of all associate degrees since 1978, more than 50\% of all bachelor's degrees since 1982, more than $50 \%$ of all master's degrees since 1987 , and perhaps most prominently, women have earned more than $50 \%$ of all doctoral degrees since 2006 (Johnson, 2016). Notwithstanding these trends, men continue to assume the majority of high-level leadership positions at postsecondary institutions, including the presidency and vice-presidency among others (Parker, 2015). Considering the rate at which women are obtaining degrees and seeking positions in the higher education leadership pipeline, the underrepresentation of women in senior administrative positions is troublesome, especially at research institutions (Dunn et al., 2014).

Although women are more likely to hold a terminal degree than their men counterparts, a common prerequisite for high-level university positions, the higher the academic rank, the fewer women hold positions of leadership (Johnson, 2016); this representation of women as faculty, staff, and administrators in higher education is commonly described as 'the higher, the fewer,' referencing the persistent gaps in equity and access for women in higher-ranking positions at colleges and universities today (Allan, 2011). As Pasque (2014, p. 332) proclaimed, "Women need to be visible and engaged at every institution type, degree program, and level of leadership. Women need to be included into the academy and once included, embraced, not silenced.” Existing research identifies the underrepresentation of women in high-level administrative roles at colleges and universities (Airini et al., 2011; Johnson, 2016; Oikelome, 2017); yet, the literature fails to address the current and pressing reasons for this discrepancy and strategies for how it can be corrected in the future (Howard \& Gagliardi, 2017; Johnson, 2016; The White 
House Project, 2009). Additionally, there is no major research to identify the influence of this men-dominant leadership structure and how the future of higher education and land-grant universities could be impacted with more gender-representative leadership.

"Understanding social forces that have shaped and continue to shape the understanding of culture can sharpen the lenses through which we examine the status of women in higher education" (Allan, 2011, p. 3). With gender equity as a constant struggle, the understanding of the perspectives of women leaders has become increasingly important. Access for women in higher education involves gaining entrée to senior leadership positions, which typically includes challenges related to the pipeline, including educational and developmental opportunities that enable women to gain the necessary credentials required for professional advancement in the field (Allan, 2011). Kowalski-Braun (2014) whose research focused on feminism in higher education, argued that feminism and its relationship to higher education transformation should be examined through a variety of different lenses.

Researchers have explored the paths of women in STEM and agricultural fields which are common to land-grant colleges and universities, yet there is no research focusing solely on the career path for women leaders at land-grant schools outside of the STEM and agricultural disciplines (Gumpertz et al., 2017; Wolfinger, 2013). Researchers have also evaluated the leadership effectiveness of men and women and its impact on various businesses and organizations; still, there has been no direct application to the effect of majority men leadership at land-grant institutions or its relation to hurdles early in the pipeline for women to be considered for administrative roles (Clark, 2006; Ibarra et al., 2013). This research, particularly as it relates to the career pipeline at various access points, is necessary to develop data-driven strategies to correct problematic patterns in the system. 
"The academy is not yet equal or equitable; our work is not done," (De Welde \& Stepnick, 2015, p. 1). The current system of higher education relegates women and continues to perpetuate cumulative oppression across generations (Pasque, 2014). Though available research suggests progress towards a more equitable representation of women in higher education leadership, there is a growing need for more research to investigate the gaps across intersecting identities and to identify factors that impede or accelerate the path (Allan, 2011). In response to these convergent calls, this study explores the opportunities and barriers to senior leadership positions in academic and non-academic tracks at a land-grant institution.

\section{Statement of the Problem}

As a result of outdated and discriminatory structures, the culture of patriarchy within land-grant institutions and a lack of priority for gender-diversifying higher education leadership, women face significant barriers in many phases of career trajectories that could otherwise support their career progression to serve in administrative leadership positions. At many institutions, these barriers include discriminatory and restrictive policies, a patriarchal culture centered around men's success, and the combination of limited resources and unrealistic expectations for women to progress professionally at higher education institutions in staff, faculty, and administrative levels (Ballenger, 2010; Hill \& Wheat, 2017; Kaplan \& Tinsley, 1989). Due to these and other barriers, land-grant colleges and universities operate with a mendominant leadership team, and by maintaining this status quo, are limiting the possibilities for the institution, its students, and their states.

Though evidence indicates that the pipeline for leaders in higher education is preparing more qualified women than men, there are consistently fewer women than men in high-level faculty and leadership positions (Johnson, 2016). Additionally, men out-earn women by almost $\$ 14,000$ yearly at public institutions and men earn more than women at every rank in every 
academic discipline at four-year colleges and universities (Johnson, 2016). Studies surrounding women's mentorship, support systems, and methods in a patriarchal workplace and society are accessible and related to this topic, especially those including the reflections of women who have attained high-level leadership roles (Bianco, 2016); however, the available research does not easily differentiate between the career paths of women in faculty and academic roles versus those in non-academic positions, or women who have experience in both.

In addition, there is no significant research related to career paths for aspiring women leaders at land-grant colleges and universities about the barriers, culture, and tactics employed to access and succeed in high-level leadership positions at such institutions. Given these practical and research problems, there is a need for research on the current landscape for aspiring women leaders across academic and non-academic pathways, and for identifying the barriers and supports within these dual tracks that influence women's ascension to senior leadership positions at land-grant institutions. The current literature tends to refer to women as a monolithic group or in some cases, emphasizes only certain subgroups of women; this study, through an in-depth investigation of a single institution, allows for insights into the lived experiences of diverse leaders who identify as women.

\section{Purpose Statement and Research Questions}

This research identifies specific supports and barriers women face throughout their careers that might enable or prevent them from attaining or accessing high-level roles in academic and non-academic administration at a land-grant university. Specifically, this study investigates the following research questions:

1. What are the attitudes, behaviors, and/or tactics that women in senior-level administrative roles employ to successfully attain a high-level position? 
2. What are the policy, structural, cultural, and behavioral influences that preclude women from advancing to leadership positions at a land-grant institution?

3. What are the attrition points and positive influences in the career pipeline identified by women?

\section{Theoretical Framework}

This study utilizes feminist theory and role congruity theory to inform the methods design, collection of data, analysis, and reporting. Using feminist frameworks and womancentric perspectives allows for intentional consideration of alternative ideas, values, and stances that can advance education and solve pressing issues (Allan, 2014; Marshall et al., 2017; Teague \& Bobby, 2014). Engaging in the discourse of gender norms and binaries can be prevalent in leadership rhetoric and the creation of policy; approaching such discourse with a critical lens can serve as a starting point to break down other gender structures that have been historically entrenched in higher education (Khwaja et al., 2017).

\section{Feminist Theory}

With a broad range of theories rooted in research, feminism involves a unique set of practices and perspectives that affirm differences among women while embodying an aspiration to promote justice and well-being of all women (DeVault \& Gross, 2012). Feminist theory informs an understanding of power, gender-related politics, and sexuality, and the impact of each on the advancement of women's rights, interests, and place in society (Komter, 1991). In this study, feminist-based research tactics and theory are utilized to maintain a strong focus on the advancement of women and the effective use of the research participants' vulnerability and storytelling to inform findings that contribute to advancement.

Feminist theory and feminist activism are crucial to constructing an understanding of everyday experiences in an academic organization (Pullen \& Rhodes, 2015; Wolf, 1992). 
Founded on the observation that gendered inequality can shape all aspects of social and economic life, feminism encourages researchers to explore how patriarchal social structures such as hegemonic masculinities and neoliberal capitalism, repress and exploit women in every facet of their life and career (Bell et al., 2019). At a basic level, feminist theory acknowledges that the relationship between men and women has always been both unequal and oppressive, and with that, the recognition that societies have operated in a patriarchal context with a system that allows men to dominate women and their lives (Komter, 1991). Social institutions in society have been characterized throughout history to be dominated by men, including political systems, economy, family, and religion, all of which can relate to the institutional environment of higher education (Komter, 1991).

Feminism exists in many forms and therefore, cannot be simply categorized in any one context as it includes the challenges faced by women of all backgrounds to secure a social, economic, political, and overall valued place in society (Gardner, 2006). "While there is diversity of experience in everyday life for both women and men, the dominant gender regime that exists today in virtually all societies is sexist in ways that result in the systematic marginalization, oppression and exploitation of women," (Bell et al., 2019, p. 8).

An important aspect of feminist theory is the focus on applying knowledge to advocate for fair treatment of all women by breaking through the social stratifications of being a woman and the social conditions that oppress and disadvantage them (Allen \& Jaramillo-Sierra, 2015). In this sense, utilizing feminist theory pushes back against traditionally men-dominated fields, including education. Challenging the idea that gender gaps in leadership placement exist because of specific characteristics can clarify the importance of breaking down barriers in gatekeeping processes or management (Marshal et al., 2017). 
Feminist theory centers around the uneven division of labor between men and women related to human ecology and how that can impact society and culture. More specifically, because women are typically responsible for reproductive labor in society, they have less access to producing work for the economy (Chafetz, 2004). This pattern has the potential to give men more access to the elite level of society, and with that, more control of that elite level, which inversely blocks access for women to follow suit (Chafetz, 2004). Further, as women lose opportunities for access to thrive in the economy, the pattern of gender stratification will only continue to grow (Chafetz, 2004).

Feminist research, then, should be aimed at producing research 'for women' (Letherby, 2003). A feminist framework recognizes that women's perspectives have long been silenced, encouraging feminist researchers then to listen intently for gaps and absences in women's narrative storytelling to consider what meanings might lie beyond the verbatim articulation (DeVault \& Gross, 2012). This study leans on feminist theory to position perceptions of women in leadership roles across many disciplines in the university setting while observing it as a critical lens to analyze gender inequality in the pipeline for women leaders in higher education.

\section{Role Congruity Theory}

Grounded in the constructs of social role theory regarding gender roles and differences in behavior, role congruity theory expands to a complete breakdown of the congruity between gender roles by identifying integral factors and processes which influence congruity perceptions and their consequences (Eagly \& Karau, 2002). Role congruity is a useful framework for this study as it helps to illuminate agentic and external influences that influence women's advancement across academic and non-academic pipelines:

A role congruity theory of prejudice toward female leaders proposes that perceived incongruity between female gender role and leadership roles leads to two forms of 
prejudice: (a) perceiving women less favorably than men as potential occupants of leadership roles and (b) evaluating behavior that fulfills the prescriptions of a leader role less favorably when it is enacted by a woman. (Eagly \& Karau, 2002, p. 573)

Through this lens, it is known that attitudes can be less positive toward women leaders and potential women leaders than those attitudes towards men, and a more difficult path exists for women to become leaders and achieve success in leadership positions (Eagly \& Karau, 2002). The perceived incongruity between women and leadership roles is likely reinforced as disparity between women's gender and the organization's gender-typing increases (Funk, 2019). Gender typing, or the presence of gender in processes, practices, traditions, and distributions of power, can predict what types of people, specifically men versus women, might be hired by an organization (Acker, 1990).

Considering the degree to which women are associated with a relative lack of agency, a double standard becomes a barrier as women must then perform better than their men counterparts to be considered competent (Eagly \& Karau, 2002). When individuals assert a stereotype about a specific social group that is inconsistent with society's traditional attributes seen to be required for success among those social roles, there is potential for prejudice to exist and prevail (Eagly \& Karau, 2002). For women leaders specifically, this prejudice can arise before women enter a leadership role, sometimes preventing them from access.

Varying definitions of leadership roles can cause a significant incongruity between the women-based gender roles and leadership expectations (Eagly \& Karau, 2002). As Eagly and Karau (2002, p. 576) explained, "the more agentically a leader role is defined or the more completely women fulfill its agentic requirements, the more likely such women are to elicit unfavorable evaluation because their behavior deviates from the injunctive norms of the female gender role." If women are perceived better when they work and spend time in environments that 
correspond with their gender, and if they are perceived less favorably in organizations that are incongruent with their gender, one could assert that women managers in masculine-oriented organizations will consistently be evaluated poorly when compared to their men colleagues (Funk, 2019).

The discrediting or discounting of a woman's performance based on a masculine benchmark can snowball over the course of a woman's career. Through the lens of role congruity theory, women and men in decision-making roles and positions of power should be cognizant of the effects of perceived gender incongruity and its potential to affect a woman's career trajectory (Funk, 2019). As with role congruity theory, feminist research is especially attentive to structures of an organization as it relates to language, talk, and discourse; people's stories are shaped by formats available to them, reflecting the perspectives and values of their environments (DeVault \& Gross, 2012). The researcher designed this study with role congruity theory of prejudice towards women leaders front of mind as a point of understanding, probing, and curiosity.

\section{Limitations}

When conducting feminist inquiry, researchers must be mindful of the unique differences that exist among women, ensuring that when speaking on behalf of women, they are speaking only on behalf of some women (DeVault \& Gross, 2012). With a history characterized by struggle, resistance, and conflict, feminism requires attention by researchers to adapt to new theorizations of the field while also acknowledging the trajectory of the feminist movement and the theorists, writings, and research practices that have led to its advancement (DeVault \& Gross, 2012). For observants who have long been steeped in their professional disciplines and who participate in qualitative studies within those professional domains, it can be exceedingly difficult to hold their own perspective in abeyance; it is not uncommon for individuals in these scenarios to rely on sharing common-sense assumptions about their experience in the field 
(Taylor et al., 2015). For this study specifically, a limitation must be acknowledged that some participants, through their research, professional leadership development, or experience, may be aware of existing research and ongoing patterns related to the experiences of women in the higher education workplace and therefore, more comfortable or knowledgeable when discussing them.

In narrative inquiry, qualitative researchers might also find themselves learning of an experience or a perspective that exists in a much broader context in which they do not have the adequate background or knowledge to completely understand (Lichtman, 2014). When writing, representing, or speaking for others, researchers represent them in certain ways as they are presenting their needs, goals, and situations; while this is the nature of feminist research, which studies women and the perspectives of their experiences, this retelling cannot be a pure reflection of all reality (Young, 2003). Further, a limitation exists in that the researcher's interpretation or retelling may not fully capture every informant's exact intention.

Maintaining the confidentiality of the participants and their stories to mitigate the risk of identification, retaliation, or retribution contributed to the limitation of this study. Participant data were shared in small segments, linked to a pseudonym when it was safe or appropriate to do so. For many participants, their title, department name, or age would immediately reveal their identities as they are either the only woman who met the study's recruitment criteria in that unit, the youngest woman in that unit, the only person with that specific title, or any combination of those factors; in extremely specific instances, the pseudonym was intentionally omitted. This process of utilizing pseudonyms and removing recognizable data limits opportunity for establishing patterns and developing deeper transferability.

An embodied perspective, one that is acutely linked to social locations and positions within a community, reinforces how researchers' social positions influence the questions asked, 
who is approached in the field, how fieldwork is conceptualized, and how findings are analyzed and reported (Naples, 2003). Because the researcher has attended and worked for the institution featured in this study, there is potential for previous knowledge to inform her perspective. This could include, but is not limited to, elements of consideration related to the cultural landscape, institutional history, and professional network. Acknowledging that outsider status and insider status are not fixed, static positions, it is essential to note that the researcher's position is ever shifting and permeable based on social locations (Naples, 2003).

\section{Significance}

Despite the number of women obtaining degrees at varying levels and contrary to the number of women on career pathways to leadership at land-grant institutions, the route for women to obtain administrator roles is unclear. There is a significant gap in the existing literature as it pertains to the experiences of women who seek top-level leadership roles at land-grant institutions and even less regarding the experiences of those women who do reach that level. In addition, there is limited research related to the trajectory of women in non-academic leadership positions in higher education, especially those who work in the sectors of enrollment management, finance, law enforcement, strategic planning, general counsel, development and advancement, alumni relations, and extension services.

Studies such as this are increasingly important to broaden the understanding of women's experiences as they aspire to leadership positions in the collegiate setting and if or when they obtain those positions, how they succeed as a gendered minority in such roles. Research in this form can help universities to better understand the context of aspiring women leaders and create clearer pathways for women, and other underrepresented groups, to access positions of leadership. Such research can better inform the nuanced experiences of women whose intersecting and potentially minority identities impact their career pathways in this environment. 
It is the intention that this study will serve as a benchmark to help land-grant and similar institutions create more inclusive, accessible climates for women to lead in, ultimately promoting an institution that is continually adapting with the society to meet the needs of its students, faculty, and staff. In addition, this study should serve as a resource for women in regard to anticipating challenges along career higher education leadership paths and presenting strategies to employ as they navigate pitfalls and encounter ongoing gender bias.

\section{Definition of Key Terms}

- Feminist research: surrounds the subject matter for issues of potential importance to women and using women's standpoints as points of departure for dedicated research (Taylor et al., 2015).

- Gender bias: the propensity to consider a person on the basis of his or her gender that can be reflected in attitudes and behaviors founded on stereotypical beliefs about the sexes rather than evaluating a person's individual abilities and experiences (National Center for State Courts [NCSC], 2006).

- Intersectionality: the relationships among multiple dimensions of social relations and subject formations related to one's identities (McCall, 2005).

- Land-grant institution: as defined by the APLU (2019), "a land-grant college or university is an institution that has been designated by its state legislature or Congress to receive the benefits of the Morrill Acts of 1862, 1890, and 1994. The original mission of these institutions, as set forth in the first Morrill Act, was to teach agriculture, military tactics, and the mechanic arts as well as classical studies so members of the working classes could obtain a liberal, practical education." 
- Microaggression: a form of common discrimination that can be rooted in bias, often signaling disrespect and directed at individuals in positions of less power (McKinsey \& Company, 2019).

- Mid-Atlantic University (MU): in order to best preserve the confidentiality of the participants' identities, this pseudonym is used throughout the study to directly replace the name of the land-grant institution where the research was conducted. All current and historical data related to Mid-Atlantic University is information regarding the study's institutional subject, including its programs, leadership, and finances.

- Woman/women: for the purpose of this study, woman and women are used to describe individuals who identify with the gender of a woman, unrelated to their physical anatomy or their born sex.

- Women leaders: in some context, using the phrase "woman leader" can counteract feminist advocacy by implying the woman cannot simply be considered as "leaders"; that is not the intended usage in this study. For this study's purpose, and throughout the literature referenced in Chapter Two, the phrase "woman leaders" is intended to denote women who hold positions of power in the workplace, and in this instance, within higher education environments. It is meant to identify one's reference to a woman in a leadership position as opposed to a man in that same type of leadership position. The term leaders is used interchangeably in this study, as it is throughout the existing literature, with the following terms: senior leaders, high-level leaders, university leadership, and administrators.

\section{Conclusion}

With the increasing presence of women in mid-level leadership roles and the opportunity for many college presidents to retire within the coming years, it is expected that women will 
change the discourse, followed by institutional cultures, and eventually, the larger society as it relates to outdated gender-specific trends (Khalid, 2016). As Eagly and Carli (2007, p. 11) said, The best leadership is found by choosing leaders from the largest pools of talent, and that includes women. Opening doors for women fosters equal opportunity and can help a society to allocate its human resources optimally. With excellence in short supply, no group, organization, or nation should tolerate the losses that follow from unfairly restricting women's access to leadership roles.

The actions of women leaders will bring about change in their organizations and within our culture, contributing to the removal of barriers that have and continue to obstruct women's paths through the labyrinth that is their route to leadership positions. (Eagly \& Carli, 2007)

For many years, society has attributed gender to leadership, outlining ways in which men and women are expected to lead, aligning with the stereotypes associated with their identities; as society evolves and new research emerges to prove that these dichotomies are becoming obsolete, the practices of applying them must also become a priority to end (Chilwniak, 1997). To analyze women's career advancement in higher education, the conversation and associated actions should focus on conceptual models within an organization's practice, culture, and boundaries, especially when considering the current lack of feminist influence on organizational norms (Eddy \& Ward, 2015). At this time, the research on women's experiences leading and aspiring to lead land-grant institutions is sparse, especially for administrators and non-academic employees on career leadership paths. This study bridges these gaps in the research to better understand the experiences of women administrators at a land-grant institution.

Chapter Two will provide an overview of the climate for women leaders in higher education, including an exploration of helpful metaphors, related theoretical concepts, and current research pertaining to women in leadership in higher education. The chapter will also 
discuss the known barriers for women seeking high-level leadership roles within universities as well as an overview of the institutional context for the study. 
Chapter Two: Literature Review 
In the past five years, society has seen more women rise to the highest levels of companies than ever before, and research indicates that organizations are starting to acknowledge the value of having more women represented at the leadership level (McKinsey \& Company, 2019). Despite progress at senior levels where the representation of women has increased from $17 \%$ to $21 \%$, women continue to be underrepresented at every level; reinforcing the notion that parity is still decades away, barriers for women in professional environments persist in the form of unfair evaluation criteria, partial and imbalanced systems, unconscious bias, and a lack of prioritization for their advancement (McKinsey \& Company, 2019).

Within higher education, women focused on obtaining leadership roles are no longer encountering a glass ceiling, but rather, a complex labyrinth with roadblocks and barriers that must be navigated with precision (Eagly \& Carli, 2007; Reis \& Grady, 2018). The uneven dynamic of gender equity in higher education over time has left barriers that still exist, though partially hidden by the increasing number of women students and the number of women entering the faculty ranks each year (Khwaja et al., 2017). Current research on gender inequality in the organizational context focuses on structural barriers and access to promotion as well as the gendered behaviors that have become internalized through socialization (Ballakrishnen et al., 2019).

Women's paths to university presidencies and other high-level administrative roles are noticeably ridden with gender-specific challenges and conspicuous barriers (Cook, 2012). Less likely to have children and less likely to be married, women presidents maneuver a different and distinctive political climate to obtain positions of leadership in the college setting; as more presidents are selected from external environments with a significant amount of executive leadership, women are less likely to be considered for such roles even though women presidents 
are more likely to hold a doctorate than presidents who identify as men, a common prerequisite for consideration (Cook, 2012).

Throughout history, many women have lacked a formal sense of authority over others and with that, no control of resources, causing them to find alternative solutions to accomplish their work; as it turns out, these behaviors and in a sense, this tactic for survival, have been highly successful in leadership and managerial settings (Rosener, 1990). Women will not be able to fairly compete and fully succeed until "they are also given opportunities to increase their selfawareness, emotional intelligence, ability to learn under never-before-encountered tasks and situations, mechanisms for self-regulation and control, which are all key ingredients of effective leadership regardless of gender," and are often experiences provided to men (Gipson et al., 2017, p. 57).

For a woman in a political career, the consciousness of gender's impact on their career will be a part of daily life, arising in new ways every day (Mandel, 2003). Though women leaders typically enact effective leadership styles more often than men and are associated with many successful organizations, there are remaining prejudicial barriers and manifestations of gendered discrimination that present challenges specifically for women (Eagly, 2007). Women are still the minority in terms of leadership representation; the woman will continue to be a representative for the entire gender when speaking out on certain issues and the need to put a woman in the room or at the table will continue to impose on her schedule and put added stress of proving women's capabilities back on her (Mandel, 2003). Institutions face a need to create opportunities and address the structural barriers and issues that negatively influence the job expectations and the experience of women at every level (Eddy \& Ward, 2015).

This chapter will discuss the current climate for women leaders in higher education amidst an inconsistent trajectory, followed by an overview of the challenges and barriers 
including an examination of gender bias and expectations, board control and gatekeeping processes, barriers related to the faculty and staff experiences, and the impact of mentorship, networking, and leadership development. The chapter concludes with a discussion of women's leadership at land-grant institutions, focusing on the current landscape at Mid-Atlantic University.

\section{Key Concepts}

Despite current efforts to eradicate gender-based discrimination in the workplace, inequity for women persists at public research universities, many of which are land-grant institutions (Rabovsky \& Lee, 2018). While navigating organizations and workplace culture, gender is continually operating as a "background" identity, informing the organizational processes, institutional beliefs, and unique individual choices (Ridgeway \& Correll, 2004). Organizations like colleges and universities are gendered through processes of practical work activities and organizational logic (Acker, 1990). This organizational logic includes job responsibilities and the expectations of women that exceed those placed upon men in the higher education workplace (Broido et al., 2015).

To better conceptualize the experiences and gender discrimination of women in organizational leadership roles, researchers lean on a series of metaphors that illuminate some barriers, challenges, and at times, recommended solutions.

Ceilings, walls, sticky floors, and glass slippers are holding women back from advancing to leadership positions, leaky pipelines predispose them to drop out, labyrinths make their career paths particularly difficult and complex, and glass cliffs make their lives risky and precarious once they have made it to the top. (Brückmuller et al., 2013, pp. 7-8)

Many have become familiar with the phrase 'glass ceiling' which for almost 40 years, has been used to refer to the invisible, systemic barrier that consistently blocks women from ascending to 
senior leadership positions; instead, today's working women are not seeing the glass ceiling as the biggest obstacle to career progression, but rather, a barrier in their first steps to reach the manager level, a concept commonly known as the 'broken rung' (McKinsey \& Company, 2019). For every 100 men promoted to the manager level, only 72 women are promoted and hired at the same rate, implying that the broken rung is actively preventing women from advancement beyond entry-level positions and reinforcing a long-term impact on the talent pipeline (McKinsey \& Company, 2019).

These metaphors, used to illustrate workplace gender discrimination, have been proven to be exceedingly powerful in illuminating the experiences women face in organizations; by adding language and drawing attention to the concept, such metaphors can allow for increased communication and understanding of the lived experiences of women in similar situations (Brückmuller et al., 2013).

\section{The Leaky Pipeline}

Even though women hold the majority in the career pipeline to be traditionally qualified for higher education leadership roles, they are still vastly underrepresented in higher education administrative leadership positions (Gangone \& Lennon, 2014). The overall underrepresentation of women leaders, especially in academic administration, suggests that masculine norms and practices function to dismiss or exclude women (Dunn et al., 2014). Though the number of women at lower academic ranks is slowly growing, the pipeline upwards continues to leak, resulting in very small matriculation at the top (Bilen-Green et al., 2008).

The issue is not that there are too few women adequately prepared to fill the positions; instead, the pipeline is continuing to prepare and accept women at a greater rate than men (Johnson, 2016). Women move through organizations at an inconsistent pace, often experiencing a limited narrowing of opportunities as they progress closer to the top (Reis, 2015). In fact, the 
higher the academic rank, the fewer women there are represented in leadership roles (Johnson, 2016). Additionally, the percentage of women serving as the Chief Academic Officer has declined from 2008 to 2013 at public research institutions (Johnson, 2016).

The preparation for higher education leadership positions does not always occur organically or without identity-related challenges along the way, and the competence of women leaders to supersede identity-based barriers does not eradicate the need to actively foster diversity and fair representation in higher education leadership (Oikelome, 2017). In many cases, the university structures, systems, and processes hinder women from advancing in higher education leadership roles in the first place (Airini et al., 2011). Those systems then create environments where women are not welcome to participate in conversations, reinforced by mencentric cliques and norms that perpetuate isolation of the women leaders present (Bilen-Green et al., 2008). Women in various sectors of higher education are continuously impacted by socially constructed gender roles in an environment that is intended to be a safe space for individuals to learn and grow while experiencing the diversity around them (Oikelome, 2017).

Women in higher education leadership pipeline lack the support that historically has been able to bring about significant change (Kellerman \& Rhode, 2014). This underrepresentation has resulted in a waste of talent for women in the career pipeline. While women possess potential to be transformative leaders in higher education institutions, it is necessary to re-socialize the system and prime women leaders to help meet the current challenges of the industry (Dunn et al., 2014). In 2013, the Colorado Women's College called on public institutions of higher education to pay particular attention to the declining number of leaders in the university setting; among all public sectors, higher education was the only entity where the rate of women leaders was declining (Johnson, 2016). 
Applying career pipeline theory in higher education suggests that the more women students, the more women in lower and mid-level positions, the more women leaders will eventually rise to the top (Kellerman \& Rhode, 2017). Application of this theory then brings about the notion that the pipeline for women in higher education leadership though swelled, is leaking. Among several barriers creating this phenomenon are gender bias and related expectations, masculine-based leadership norms, patriarchal culture, caregiving related challenges, and heightened difficulty for women of varying intersectional identities.

\section{The Glass Cliff}

Though research indicates that women in the workforce are shattering glass ceilings to access senior management roles, there is limited research regarding their experiences once they continue in those positions (Sabharwal, 2015). In some cases, women break through the glass ceiling through appointment to upper-level leadership positions, only to learn that the position is perceived to be more precarious and as some would define it, a glass cliff (Ryan \& Haslam, 2005). By definition, glass cliffs represent the concept that "women may be preferentially placed in leadership roles associated with an increased risk of negative consequences. As a result, to the extent that they are achieving leadership roles, these may be more precarious than those occupied by men" (Ryan \& Haslam, 2005, p.83). Put simply, glass cliff appointments denote situations when women are appointed under circumstances different from those of men, often in times of crisis and risk (Peterson, 2016).

"Not only are women more likely than men to accept and occupy positions that have a higher risk of failure, they are less likely to be given second chances after a failure," (Pasquerella \& Clauss-Ehlers, 2017, p.11). Such positions, often offered to women over men, are dubbed glass cliffs due to their association with more danger than other similar positions and the pretense that these positions could set them up for failing, ultimately leading them over the edge 
(Ryan \& Haslam, 2005). Frequently arising as a result of psychological and social constructs rooted in sexism, glass cliffs become a predictor that women in those roles are more likely to leave an organization (Sabharwal, 2015). In some instances, women who reach a glass cliff position may "be differentially exposed to criticism and in greater danger of being apportioned blame for negative outcomes that were set in train well before they assumed their new roles," (Ryan \& Haslam, 2005, p. 87). The glass cliff phenomenon is in itself an extension of the glass ceiling; once women crack the ceiling to access a senior role, they are still unable to exert authority in the same way as men (Sabharwal, 2015).

This concept can also refer to the pretense of women rising to positions of organizational leadership in crisis, as opposed to men rising to similar positions when the organization is in a time of prosperity (Bruckmüller \& Branscombe, 2010). As Kark and Eagly (2010, p. 449) asserted, "Not only are women perceived as less suitable than men to higher level positions in general but also they are regarded as more suited than men to manage organizations that are failing and, thus, in organizational crisis." Naturally, the risk of organizational and personal failure is high for individuals who accept such positions during challenging times (Kark \& Eagly, 2010).

Another factor contributing to these phenomena involves longstanding stereotypes of gender and leadership, leaning on the depiction of a man as the historical representation of a strong and steadfast leader (Bruckmüller \& Branscombe, 2010). Gender-based stereotypes and the preference of some men to avoid crisis-related positions have been found to reinforce the glass cliff concept; when women are appointed to more precarious leadership positions, it is often not because they are seen to have earned them, but because men no longer seek them or enjoy them (Bruckmüller \& Branscombe, 2010). In organizations led predominantly by men, the glass ceilings and glass cliffs could be explained by status quo bias, the notion that as long as a 
company is performing well, there should be no perceived need to change; this would imply then that if the company has been led primarily by men and is performing well, there is no perceived need to interrupt by promoting women (Bruckmüller \& Branscombe, 2010). Applying the concept of the glass cliff to higher education is a vital lens through which we conceptualize the slow rise of women in high-level positions by emphasizing the context of women's participation in higher education leadership and drawing attention to the problematic nature and heightened expectations of the positions they might be appointed to (Peterson, 2014).

\section{The Labyrinth}

Arguing that reliance on the glass ceiling metaphor is outdated, Eagly and Carli (2007) noted that there is not one absolute barrier at the high level of an organization, nor are the barriers women face difficult to detect or potentially unforeseen. The glass ceiling metaphor fails to confirm the diverse strategies that women must use to reach positions of leadership, it assumes that all women can eventually become leaders, implies that women have equal access to positions at all levels, and ignores the potential for strategic problem solving to possibly create clearer paths for women (Eagly \& Carli 2007). Instead of encountering a glass ceiling, women navigate a complex labyrinth; some women are finding ways to obtain access to higher levels of organizational leadership, but to do so, must commandeer a labyrinth of twists and turns, detours, and at times, dead ends (Eagly \& Carli, 2007). Rather than a formerly implied barrier at the top, women are now facing multiple levels of difficulty on their career trajectories while men continue to follow a straighter path with fewer obstacles (Bruckmüller et al., 2013; Eagly \& Carli, 2007). Through this lens, the unequal representation of women in executive positions is a result of many challenges and obstacles (Eagly \& Carli, 2007). Among these obstacles for women are differential access to resources, varying domestic responsibilities, differences in support networks, and ongoing gender stereotyping and prejudice (Eagly \& Carli, 2007). 
It is recognized that women face more challenges in navigating this labyrinth and in many cases, need more determination than men counterparts to achieve their professional goals; this instance mainly illuminates the need for targeted guidance, mentoring, and support to maintain ambition and adequately showcase women's competence (Ellemers, 2014). A woman's route to leadership "wends through a labyrinth, where they find themselves diverted - sometimes by doubts about their competence, sometimes by doubts about their warmth, and sometimes by resentment of their very presence," (Eagly \& Carli, 2007, p. 117).

As evidenced by the application of these metaphors to conceptualize women's directions to leadership in academia, the pathways are unclear. The following section outlines a number of the unique challenges women face and how they manifest in the working environment of higher education.

\section{Challenges for Women Administrators in Higher Education}

It is no secret that women remain vastly underrepresented at the high organizational levels in the United States of America (Gipson et al., 2017; Paustian-Underdahl et al., 2014). This underrepresentation has resulted in a waste of talent for women; while women possess potential to be transformative leaders in higher education institutions, there is no time like the present to re-socialize the system and prime women leaders to help meet the current challenges of the industry (Dunn et al., 2014).

While there might not be a singular cause of gender disparity in the workplace, there is research to prove that the disadvantages for women accumulate, often becoming insurmountable barriers for individuals in the workplace environment over time (Ellemers, 2014). Research has shown that the challenges women face when being considered for high-level leadership positions often result in a culture that ultimately prevents women from advancing to the highest role in organizations at the same rate as their men counterparts (Gipson et al., 2014). As women are 
constantly encouraged to seek leadership positions proactively, there must first be action towards addressing the policies and practices of organizations that communicate incongruity between how women are seen and how desirable leaders are defined and viewed by society (Ibarra et al., 2013). As Pasquerella and Clauss (2017, p. 10) explained, "Hidden biases that result in a lack of opportunity for women to move up the ladder into leadership roles, the active discouragement of women, and differing expectations imposed on men and women within academia take a toll on efforts to increase the number of women presidents," implying impact on women throughout varying levels of leadership in higher education and beyond.

\section{Gender Bias and Expectations}

Gender and the socially constructed differences between men and women and their expected beliefs and identities are present in every organization; gender-based sexualized assumptions shape the situations of power for women and men in unique ways (Acker, 2006). Hierarchical organizations especially are gendered and racialized, often more so at the top as leading hierarchical positions are occupied by white men in the United States (Acker, 2006). Institutional practices may appear to be equitable or gender-neutral at first glance, but in reality, are perpetuating ongoing gendered practices (Broido et al., 2015). In many organizations, there is a sustaining sense of second-generation bias embedded into the organizational norms; when left unidentified, this second-generation bias puts women at a severe disadvantage (Ibarra et al., 2013). For example, recent research has found that $91 \%$ of men and $86 \%$ of women show at least one form of clear bias against women in areas of politics, economics, education, and women's reproductive rights; on a global scale, $50 \%$ of men believe that men should have more right to a job than women should (United Nations Development Programme, 2020). One of the greatest challenges for underrepresented women in leadership roles is the persistent and often unconscious bias, leading to the silent deduction that women lack the competence of a man 
equivalent and therefore, they must work harder to reach the same results as a man (Ellemers, 2014; Kellerman \& Rhode, 2017).

\section{Implicit Bias}

Subtle gender biases can be defined as nonreflective personal acts of exclusion that play out in social interaction, typically disproportionately disadvantaging women and reinforcing institutionalized gender norms and practices (Bird, 2011). "People tend to rely on implicit gender stereotypes to make inferences about the ambitions, characteristics, and priorities of men and women in organizations. In this way, they unwittingly evaluate men and women at work differently, causing women to perceive fewer advancement opportunities than men" (Ellemers, 2014, p. 51). It is not uncommon for this process to undermine inspiration and productivity, causing women to opt-out (Ellemers, 2014).

“Although prejudicial attitudes do not invariably produce discriminatory behavior, such attitudes can limit women's access to leadership roles and foster discriminatory evaluations when they occupy such roles," (Eagly, 2007, p. 8). When women assert power in many organizational situations, they violate conventional stereotypes of subordination to men and face being unfairly labeled as "witches" or "bitches" (Acker, 2006). In the educational setting, women continue to face discriminatory practices that hamper development, ingenuity, and achievement; it is not uncommon for them to receive messaging that they are more lovable and acceptable when they have little or no power (Trahan \& Growe, 2012). Sometimes, this bias can lead to hindered feelings of confidence or self-worth; due to the existing gender bias in some working environments, women believe that they must meet more qualifications to be hired for a new position than a comparative man counterpart (Mohr, 2014).

While there are policies to address bias, and in some cases specifically gender bias, these policies are typically blank policy instruments that leave the masculine definitions of leadership 
resolutely unargued (Marshall et al., 2017). To support women's access to leadership positions, Ibarra, Ely, and Kolb (2013) suggested three main tactics: educate women and men about second-generation gender bias, (2) create safe environments to support transitions to bigger roles; and (3) anchor women's development efforts in a sense of leadership purpose rather than in how women are perceived.

\section{Gender-based Expectations}

Historically, the representation of women has negatively implied that she must be docile, passive, conventional, and acquiescent, reinforcing their status as second-class citizens in a patriarchal society where their place is second to men (Trahan \& Growe, 2012). In most roles, women are expected to be supportive, gentle, understanding, and acting out of service to others, deriving a sense of self-esteem from helping others (Rosener, 1990). While women are anticipated to be cooperative, supportive, vulnerable, and emotional, men must appear to be competitive, strong, tough, decisive, and in control (Paustian-Underdahl et al., 2014; Rosener, 1990). Generally, women in leadership positions believe that people perform most efficiently when they feel good about themselves and their work, leading them to create situations that help contribute to this feeling and therefore improve productivity (Rosener, 1990).

To address this complex environment and the expectations associated with the double bind for gender stereotypes, professional women employ a wide range of navigational tools including the choice to accept organizational pressures while adapting behaviors and changing expressed opinions to pass as someone they are not (Ballakrishnen et al., 2019). However, some women utilize intentional invisibility by staying behind the scenes and placing high value on communal and collaborative work, often limiting their opportunities for advancement at the sake of avoiding conflict and maintaining stability (Ballakrishnen et al., 2019). In either scenario, women are adapting above and beyond their call of duty to fit in and stay in a masculine- 
dominated organizational workforce. When an organization falls into a pattern of assumed gender schemas, the workplace and in some ways, the campus, becomes an environment where women are marginalized and their contributions are devalued (Broido et al., 2015).

\section{Masculine-based Norms}

In the United States, women are praised for their leadership skills and professional effectiveness while simultaneously portrayed as inferior to men and looked over for leadership positions (Eagly, 2007). As Sulpizio (2014, p. 103) explained:

One of these gender biases is the male-as-normative model of leadership, where leadership behaviors are aligned with masculine associated traits such as decisiveness, assertiveness, and competition. The qualities associated with being female — relational, emotional, and passive — are incongruent with socially accepted leadership practice. Because history has aligned many leadership-based characteristics with masculine identity, women are frequently set up to face double standards, walking the line between being assertive enough, but not too assertive at risk of being judged as abrasive (Kellerman \& Rhode, 2017).

Conceptions of leadership are constructed by society to be associated with a strong, competent, fatherly demeanor, a guardian of moral values, and a provider whose orderly strategies solve challenges and allay fears, all of which are frequently linked with masculinity (Marshall et al., 2017). In fact, most general work is organized with the image of a man in mind, one who is totally dedicated to his work and who has no responsibilities for family other than to earn a salary to support one (Acker, 2006). Behaviors and norms most associated with masculine behavior lead women to make adaptations to meet expectations of their environment, often but not limited to family-related responsibilities (Acker, 2006).

For women, the integration of leadership into their core identity can be especially challenging while establishing credibility in a society that is frequently conflicted about the mold 
for a desired and respected leader (Ibarra et al., 2013). One of the most common ways women try to fit into men-dominated organizations is by emphasizing their own depiction of masculine characteristics and leadership styles to reinforce their advantage in hopes of being considered for leadership roles (Derks et al., 2016). Often, the underrepresentation of women in top positions reinforces entrenched beliefs that support leadership by only men and, therefore cultivating and maintaining a culture of workplace gender bias that can interrupt and hinder the leadership identity development of women within the organization (Ibarra et al., 2013). Sometimes, women's ambitions are stifled by a work environment or corporate culture that suppresses their success and potential (Clark, 2006).

“Attending to women's experiences of leading within the context of higher education affords insights into the connections among gender, authenticity, and leadership" (Gardiner, 2014, p. 167). Since most scholarly work on leadership has been focused entirely on men, it is the masculine traits, patterns, and characteristics that women are held to as the standard while they navigate the men-centric environment of higher education administration (Dunn et al., 2014).

\section{Patriarchal Culture}

Among the glaring barriers within hiring and promotion for women in higher education institutions, there is also a succinct culture of secrecy in respect to earnings; women do not typically know or have access to learn about how their salaries might compare to those of men counterparts, and if they are hired at a lower rate, it is unlikely that they will ever make up the difference over the course of their career in the field (Frances, 2018). In organizations with clear hierarchies, there are gender patterns embedded into the culture, constantly created and sustained in the form of restricted opportunities due to the gendered expectations of behavior, attitude, and commitment to the role (Acker, 2006). 
Though institutionalized patriarchy and its effect may seem noticeable in certain environments and context, the bias against women's leadership is rarely useable in lawsuits and arguments (Marshall et al., 2017). Women are known to share power and information as assets rather than liabilities or scare tactics in a leadership position (Rosener, 1990). Though women who have held power in public arenas have used their position to make a difference by advancing the function and being more responsive to broad needs of the population, they cannot be viewed as bringing about the permanent systemic change or societal transformation necessary to create a new norm for women leaders (Mandel, 2003).

In addition, research has indicated that both men and women are less confident and less motivated to lead on teams where men are the majority, and those majority men teams are particularly negative environments for the women present (Born, et al., 2018). Among other factors, this is typically driven by a communal under-confidence in women to lead and a related lack of support for potential women leaders (Born et al., 2018). This and similar research reinforce the question of how organizations can balance differing needs and gendered stereotypes to create an environment of equal opportunity and maximum leadership effectiveness (Born et al., 2018).

For women specifically, achieving a work-life balance while assuming child raising responsibilities can have an asymmetrical effect, hindering opportunities for collaborative research projects and building career networks (Bird, 2011). Due to the assumed caregiving and nurturing nature, women managers quietly maintain the organizational housekeeping necessary for efficiency while men heroically solve the visible challenges (Ely \& Meyerson, 2000). For those who have never had to think about it, seeing and acknowledging institutionalized barriers that hinder women's advancement in higher education can be extremely difficult (Bird, 2011). Though well-intentioned, the above scholarship focuses primarily on white women or in some 
cases, treats women as a monolithic construct, failing to address the interesting identities of women and how they compound challenges, barriers, and oppression in the workplace.

\section{Intersecting Identities}

There is no singular story of women in the workplace; gender is one of several facets of women's identities that can impact their experiences in different environments (McKinsey \& Company, 2019). Race, class, ethnicity, ability, and other intersecting identities can complexify the experience of inequality and oppression (Acker, 2006). What holds true for white women in positions of leadership might not hold true for women of other racial and ethnic groups (Kellerman, 2003). A more nuanced understanding of women's representation in the context of higher education requires consideration of intersectionality (De Welde \& Stepnick, 2015).

Research has proven that cultural stereotypes of gender become more complex when the multiple intersecting identities of individuals are taken into account (Kark \& Eagly, 2010). As there are significant gaps in the literature regarding the experiences of women's intersecting identities, it cannot be assumed that primary and secondary research mentioned throughout this study can be directly applied to all women as the research does not clearly acknowledge how challenges and barriers manifest at the intersection of multiple identities including but not limited to: sexual orientation, ability, socioeconomic status, race, ethnicity, assigned sex, and age, among others.

The processes by which organizations are structured might be moving in the direction where inequalities are more subtle, but the white man worker positioned as a model worker remains strengthened (Acker, 2006). According to Wyatt and Silvester (2015), Black and ethnic minority individuals experience a particularly difficult path when navigating the career labyrinth, especially when attempting to access informal resources necessary for advancement, including network opportunities, mentorship, and information shared among manager-level employees. This research demonstrates that Black and ethnic minority individuals who reach senior-level 
roles have put forth significantly more effort to make sense of the informational, organizational processes in order to access that position (Wyatt \& Silvester, 2015). Along the same lines, women (and especially women of color) have found that people at all levels of their work environment, including peers, can be intolerant of their mistakes, leading women to be more risk-averse in the workplace as a result (Kellerman \& Rhode, 2017).

Black women are not acquiring positions of leadership in the academy at the same rate as individuals of other identities, and the limited number of Black women in senior-level roles reflects the notion that they are not reaching positions that allow them to create institutional and social change at comparable rates, but rather, are more likely in positions to implement policies and programs built by others (Konrad \& Pfeffer, 1991, in Glover, 2012). For Black women in the workplace, it is common to have their judgement questioned related to their area of expertise in addition to continually being asked to prove their professional competence (McKinsey \& Company, 2019). Black women are forced to constantly prove their capabilities while disproving programmed beliefs that assume an inability to excel as an instructor and a colleague (Davis \& Brown, 2017). In addition, research indicates that women with disabilities, Black women, and lesbian and bisexual women have a distinctly worse experience in the workplace compared to women in other identity groups, specifically related to equal opportunities for advancement, overall support from managers, fair and objective promotions, and support for growth and development (McKinsey \& Company, 2019). Though the phenomenon in higher education leadership is still relatively unexplored, discrimination and disadvantage can be intensified at the intersection between gender and sexual orientation as stereotypes and gender role assumptions are often pushed upon individuals who identify as nonheterosexual (Kark \& Eagly, 2010).

For Black women and Latinas, seeking promotion from entry-level positions can be exceedingly challenging; for every 100 men promoted from entry-level roles to manager roles, 
only 68 Latinas and 58 Black women are promoted in the same pipeline (McKinsey \& Company, 2019). For women of color explicitly, navigating a deep level of exclusion associated with racial and gender identity can add a significant layer of difficulty to networking and working in the higher education environment (Mainah \& Perkins, 2015). "When subjected to stereotyping based on gender and race, women of color may have a double disadvantage as managers and as applicants for managerial roles," (Kark \& Eagly, 2010, p. 449).

Women of color are often numerical tokens in the structural environments of higher education institutions (Turner et al., 2011). Feeling marginalized in the workplace can lead to lower levels of control over one's distinctiveness and belonging, which are critical factors for individuals to feel seen and appreciated (McCluney \& Rabelo, 2019). And, the descriptive aspect of identity-based stereotypes can intensely impact individuals' behavior by triggering anxiety and diminishing their desire or ability to put themselves forward as potential leaders (Kark \& Eagly, 2010).

Research indicates that Black women and women with disabilities face more barriers to advancing in the workplace in addition to receiving consistently less support from managers and experiencing less sponsorship and support than other groups of women; they are also less likely to feel as if they have an opportunity to advance, they are less happy at work, and they are more likely to leave their jobs than other women would be (McKinsey \& Company, 2019). In addition, certain groups of women are more prone to experiencing microaggressions in the work environment; lesbian women, bisexual women, and woman with disabilities are more likely to fear consequences of discussing their personal lives at work and are more likely to experience targeted degrading remarks (McKinsey \& Company, 2019). In the environment of academia, the salience of gendered realities combined with the ongoing realities of racism is inextricably linked to the overall work life experience of women and women faculty (Turner et al., 2011). 
Microaggressions and other forms of marginalization can lead to paradoxical tensions which reinforce tensions surrounding race, gender, and class in the workplace; these tensions often create conditions of visibility which impact how people are seen (McCluney \& Rabelo, 2019). Often, these conditions of visibility are gendered and racialized, reinforcing the systemic normalization of whiteness and maleness in organizations and their hierarchies; for Black women and other marginalized identity-based groups, research indicates that their career trajectories are deeply tied to structures of race, gender, and power within organizations and the broader society in ways that significantly affect their vocational experiences (McCluney \& Rabelo, 2019).

The exclusion and marginalization of Black women throughout higher education are well documented through the lens of historical and modern perspectives (Davis \& Brown, 2017). Yet, research regarding their pathways to administrator-level careers as well as the experiences of multi-racial women or women of multiple intersecting minority identities is not current or common. There is an urgent need for more substantial research to examine how intersecting identities, and especially underrepresented identities, impact a woman's career and lived experience in the higher education and broader organizational environment.

\section{Boards and Gatekeeping}

Governing boards at public institutions have the authority to hire, review, and terminate a university's president, as well as the authority to lead or participate in search processes for other high-level administrative positions (Eckel, 2019). The employment practices used to hire new employees, specifically professors, at state institutions have resulted in a pattern of fewer women employed in the most high-paid departments and divisions, revealing a horizontal exclusionary process (Hironimus-Wendt \& Dedjoe, 2015). This pattern also implies that the gatekeeping processes used to hire employees in academic roles are associated with a sustaining wage gap between men and women (Hironimus-Wendt \& Dedjoe, 2015). 
At public institutions, gatekeeping processes related to hiring, promotion, and prioritization are directly linked to the governance by boards and trustees. Men are overrepresented in important decision-making processes and on evaluating committees in the university setting, increasing the likelihood that what men take for granted and define as normal will inform the ways in which they, and others, interpret strategies for success and define accomplishments when evaluating dossiers in the tenure and promotion process (Bird, 2011). In this scenario, individuals who have not personally taken on a caregiving role and often mostly men, might fail to consider the reasons why both women and men who are primary caretakers are forced to structure their work uniquely, adapting traditional work hours and avoiding prolonged travel (Bird, 2011).

For years, governing boards in higher education have remained relatively homogeneous in nature, valuing the voices of white men in business, law, faculty positions, clergy, and politics (Kerr \& Gade, 1989). In 2010, the average public institution's board had 12 voting members, including nine men and three women (Fain, 2010). Across all public governing boards, women made up about $30 \%$, a slight increase from $28.4 \%$ in 2004 , and more than four-fifths of board chair roles were held by men (Schwartz, 2010). Though steady progress is expected in the coming years, data show that men still outnumber women in representation on public and independent governing boards by more than two to one (Johnson, 2016).

This lack of representation can serve as another barrier for women seeking administrator roles as they overcome gender bias and reinforced gender norms which may falsely denote that they are not as equipped as men for high-level leadership positions. Research has identified a positive correlation between the number of women board members and the number of women in top administrative positions in corporations; research has also identified a positive correlation 
between women board members and the number of women serving as presidents, provosts, and faculty members at colleges and universities (Schwartz, 2010).

Women are known to have a positive impact on governing boards, impacting cohesion and monitoring the management of tasks while fostering strategy development (Brieger et al., 2019). Research has shown among corporative executives and boards of directors that U.S. firms find the inclusion of women to be linked with more robust financial performance (Eagly \& Carli, 2007). In fact, building gender-diverse boards can be seen as a strategy to create strong value by establishing a norm of gender equality in the greater organization (Brieger et al., 2019).

There is a call for colleges and universities to critically examine the longstanding cultures and norms within their working environments, pushing back on the assumptions about who can and cannot be a leader and redefining how those leaders are cultivated, selected, and benchmarked. Without necessary changes to organizational culture and norms, colleges and universities could miss out on the quality talent and potential of women leaders, and others, who might not perfectly fit the formerly and assiduously defined model of success (Eddy \& Ward, 2015).

\section{Faculty}

There are formal and noticeable aspects as well as informal norms of the culture at research universities that shape expectations in the workplace and result in gendered differences in the faculty workload (O'Meara et al., 2017). Faculty members who identify as women earn less than the men-identifying coworkers at almost every type of college and university (American Association of University Professors [AAUP], 2011). At four-year institutions, the pay gap can be the largest, with women earning $20 \%$ less than their men counterparts (AAUP, 2011). 
In addition to a gap in treatment and wages, women faculty are subject to face a higher concentration of gender stereotypes, intolerance of mistakes, in-group favoritism, double standards related to men-centric norms, implicit bias against women, availability of opportunities to men only, and family conflict (Kellerman \& Rhode, 2014). Research has proven that compared to their men counterparts, women professors bear a disproportionate amount of service-related work (Flaherty, 2018; Guarino \& Borden, 2017). As women ascend to positions of authority, they face a challenge to overcome the tradition of servitude; this barrier often elicits questions of how women can execute empowering decisions while maintaining relationships and how women can take care of themselves while still having to take care of others (Trahan \& Growe, 2012).

\section{Service}

Across all disciplines, there is a gendered division of labor; as service is not easily quantified, it often goes unnoticed (De Welde \& Stepnick, 2015). Therefore, it is no secret that women regularly perform a disproportionate share of care work and emotional labor among academic units in higher education (Bird, 2011). It is not uncommon for research-intensive universities to over-recruit women faculty to serve on university task forces and committees for the sake of gender diversity, a practice that is, in and of itself, an institutional barrier for their advancement as it deprives them of valuable time to conduct research, a necessary activity for promotion (Pyke, 2015). Women are assigned more campus service and advising related tasks than men (Guarino, 2017; O’Meara et al., 2017; Pyke, 2015). Overall, when asked to engage in work-related activities or tasks, women say yes more often than men do, likely due to the pressure of women as an underrepresented party in the academic leadership ranks, pressure to say yes to avoid gender-based stereotypes, and pressure to consistently impress colleagues to prove their worth in a gendered system (O’Meara et al., 2017). 
Women faculty are bearing an unreasonably large part of the service burden by "taking care of the academic faculty" so to speak; women faculty consistently outperform men faculty, especially as it relates to internal service within the unit or organization (Guarino \& Borden, 2017). It is more common for administrators and colleagues to invite women faculty over men to be involved in teaching and campus service because typically, there is a need to add diversity to committees, there is anticipation that women will say yes, and there is a perception that women are good at teaching and service-related activities (O’Meara et al., 2017).

When women do not live up to set gendered stereotypes, such as saying yes in an opportunity to help or contribute to the welfare of others, they are perceived in a negative light which puts additional pressure on women faculty to confirm their participation when asked in the academic setting (Heilman et al., 2004). The differential in service loads between men and women in academia "points to a pervasive gender effect, transcending departmental context, suggestive of a deeper sociological difference between men and women in relation to service tasks or simply effects of discrimination," (Guarino \& Borden, 2017, p. 690). It is people within the system of higher education itself who do not regularly assess workload equity for women (Pasque, 2014). To advise women on how to best manage a heavy service load, university administration often encourages them to seek advice from senior colleagues, most of whom happen to be men who lack relatable experience regarding the same burdens and pressures (Pyke, 2015).

Since women are often expected to be more nurturing and are perceived harshly when they are not, students inherently hold women instructors to higher standards than they do with men and often rely more heavily on them for favors, advice, and service (Flaherty, 2018). Researchers have also demonstrated that students are typically more willing to seek friendshiptype relationships from women professors, setting higher standards and putting a deeper 
emotional strain on the women in academe often leading to burnout and lower job satisfaction compared to their men peers (El-Alayli et al., 2018).

Women faculty are reportedly feeling a significant emotional strain, spending more time working through student requests, serving more unhappy students, receiving lower course evaluations, and having less time to budget for research, class preparation, and additional service due to extra demands (El-Alayli et al., 2018). This burden of overwhelming service-related work and the unfair standard by which students are reviewing their women faculty can have severe negative implications in the promotion and tenure process (Flaherty, 2018).

Though most research confirming the unequal burdens of service relates to women as a monolithic group, there is a growing body of knowledge related to the phenomenon of greater service burdens falling on the shoulders of Black and marginalized, underrepresented, or minority women. As a direct result of being highly tokenized minorities at predominantly white institutions (PWIs), Black and African American women assume a heavier role than others in service, teaching, and research as faculty members (Harley, 2008). Padilla (1994) explained this phenomenon as a form of cultural taxation, describing the added burden of service-related responsibilities often placed on minority faculty members due to their ethnic or racial background. Building upon Padilla's (2014) work, Hirshfield and Joseph (2011) introduced the concept of identity taxation to identify and include the pretense for individuals of other marginalized identities (including but not limited to race, gender, and sexual orientation) to be asked to complete more academic and non-academic service related tasks as faculty members. In addition, research indicates that Black women faculty members are frequently deemed nurturing as mothering figures, often leading to expectations that exceed those of typical coworking and advising relationships (Hirshfield \& Joseph, 2012).

\section{Staff}


Though most research about the experience of women in higher education focuses on individuals in faculty roles, there is much to be said and explored regarding the non-academic staff members. In fact, most research on work life balance for women in the college setting focuses solely on tenure-track faculty, giving little to no acknowledgement of non-tenure track faculty, administrators, and student affairs professionals (Fochtman, 2011).

Current research focusing on women in student affairs merely scratches the surface of the challenges faced by professionals serving the non-academic functions of universities (Yakaboski \& Donahoo, 2011). However, it is known that the gender-based social structures of femininity and general womanhood have unique implications in the student affairs workplace, specifically reinforcing gendered practices within the functions, offices, and professions that serve nonacademic student support services (Yakaboski \& Donahoo, 2011).

\section{Women in Student Affairs}

Representation by women and other marginalized groups is especially vital in student affairs professions. As new professionals enter the field often, the staff must represent the growing diversity of student populations, and it has become increasingly important to adequately supervise and relate to individuals of diverse backgrounds (Davis \& Cooper, 2017).

For women in student affairs professions, institutional culture typically regards their roles as less important or inferior to those in academic and business affairs units, pushing career student affairs professionals into a subordinate level of institutional hierarchy (Yakaboski \& Donahoo, 2011). There is typically an expectation for student affairs professionals to work nontraditional hours, especially in fields of residence life, new student orientation, and student activities. The field of student affairs administration as a whole places extremely high and often unrealistic demands on the time and energy of its professionals, especially for a traditionally low-paying career path (Marshall et al., 2016). 
For primary caregivers, the expectation of working these nontraditional hours in student affairs roles can be a barrier to success in the position and can place additional pressure on both men and women with caregiving responsibilities (Yakaboski \& Donahoo, 2011). Likewise, current and ongoing fiscal constraints with university administration result in staff downsizing and fewer resources to work with, often adding to the burden of unfair time commitments and an extension of job responsibilities, as well as an expectation to fully commit to your role for the sake of the students and the institution (Marshall et al., 2016).

Research has indicated that there are a limited number of ascents from student affairs roles into college presidency positions despite the well-educated, practiced, and often women leaders; women administrators in student affairs do not then have the same career mobility as their colleagues in other sectors of campus (Jacobson, 2002; Yakaboski \& Donahoo, 2011). Student affairs professions and the access, patterns, and impact of gender within the sector of higher education are still relatively understudied. Therefore, there are several remaining gaps in the literature related to this subject.

\section{Outside the Realm of Student Affairs}

Women in noninstructional and non-administrative positions, including professional, clerical, technical, and related roles, are often overlooked in research and examination of the workforce issues in higher education (Allan, 2011). For non-academic staff, there are added barriers. In many institutions, classified staff lose seniority when they resign from their positions and return later, making them more susceptible to be laid off when budget cuts occur (Broido et al., 2015). Additionally, the gender gap in part-time employment at colleges and universities reflects an institutional decision to label certain types of work as less valuable, hiring women more than men to fill those roles (Broido et al., 2015). 
Acknowledging the explicit and implicit ways in which women have been oppressed is imperative in how women and women's issues are addressed in the context of higher education and student affairs (Niskodé-Dossett et al., 2011). As mentioned above, there is a significant gap in research addressing non-faculty and non-academic university staff members, especially those outside of student affairs roles including but not limited to, women who work in alumni relations, bursar and treasury services, admissions, public and university relations, government relations, athletics, healthcare, and extension.

While there may be common threads and experiences, the research focused on women faculty, deans, and academic administrators cannot be directly applied to those who work in different units across the university's operation; the nature, structure, and policies related to academic roles do not parallel those of non-academic roles in the university setting. To address gaps in the research, this study considers the unique challenges and intersecting identities that contribute to the gendered experiences of women in such positions.

\section{Leadership Development}

Colleges and universities seeking to diversify their administration are paralyzing their advancement by not prioritizing women's progression in leadership roles (Eddy \& Ward, 2015). By creating a more distinct team of diverse institutional leaders, institutions can better position themselves to meet the challenges of an evolving field while utilizing a variety of strategies, skills, and opinions (Eddy \& Ward, 2015). “Gender (including our transgender students and colleagues) should be reflected in policy, programs, and practice - not avoided as though we are in a gender-free, post-racial, or flat society in terms of class stratification - we are not," (Pasque, 2014, p. 332).

When employees believe their company to be serious about accountability, they are more likely to be happier and more likely to stay with the organization; yet only $32 \%$ of women 
believe the disrespectful behavior towards women is quickly addressed in their workplace (McKinsey \& Company, 2019). An important first step for institutions is to hold administrators accountable for acknowledging and addressing the inequities for women in the system by valuing diverse leadership and supporting programs that will help the institution move forward (Kellerman \& Rhode, 2014). These leadership development programs, especially those tailored for women, should provide opportunities for participants to explore their authentic selves while developing a capability to utilize leadership tactics associated with both feminine and masculine norms (Sulpizio, 2014). By showcasing women's leadership as an attainable process through intentional women-centered programming, participants will experience pivotal shifts in their mindsets to recognize women's capabilities to lead in both official and unofficial capacities (Sulpizio, 2014). A performance-based, all-encompassing climate is crucial for women to rise to leadership positions and in theory, for a university to reach high potential (Gangone \& Lennon, 2014).

Among these strategies, initiatives for mentorship and sponsorship are critical, especially in areas where formal relationships and connections might be lacking or departments where women might not have natural or easy access to like-minded champions (Kellerman \& Rhode, 2014). Multiple coherent leadership programs for men and women are necessary, as are various forms of women's leadership programs for distinct, and in some cases, overlapping populations of students, faculty, staff, and administrators (Keohane, 2014).

\section{Mentorship and Networking}

There is no question to the importance of mentoring relationships for women who aspire to administrative leadership roles at colleges and universities (Dunbar \& Kinnersley, 2011). Regarding the preparation and recruitment for women university presidents, mentoring is invaluable (Brown, 2005). Mentorship and multiple mentoring relationships promote the 
advancement of women through leadership positions in higher education administration and help to increase the number of women holding college and university presidencies (Brown, 2005). For many women in leadership roles at the college level, mentoring relationships are formed entirely on their own time through their own extra effort without the support from their institution (Fochtman, 2011; Hill \& Wheat, 2017).

Peer group programming and mentoring opportunities have been recommended to promote growth and combat the imposter phenomenon among women leaders in the academic environment (Huffstutler \& Varnell, 2006; Parkman, 2016). The imposter phenomenon is common for individuals, particularly women, experiencing ambiguity or change in their working environments; this psychological pattern can manifest with perceptions of intellectual fraud among high achieving women, often causing them to internalize their success and question their qualifications (Clance \& Imes, 1978). This phenomenon, which is well documented in academic environments, when curbed can create a more inviting environment for high-performing faculty and staff members at universities (Parkman, 2016). In addition, reversing the pattern can increase the retention of productive faculty and staff women (Parkman, 2016). To help alleviate instances of imposter phenomenon and build confidence in women leaders who experience it, Clance and Imes (1978) recommended mentorship that focuses on establishing empathic and supportive relationships that are non-authoritarian. These mentoring relationships can be especially beneficial for current as well as aspiring faculty and staff (Guillaume et al., 2019).

Finding mentors and gaining access to informal networks remains a common obstacle for women, often attributing this challenge to the perception and reality of "boys clubs" and "old boys' networks" (Broido et al., 2015; Searby et al., 2015). Research indicates that institutions benefit when investing in formal and informal mentorship programs (Jackson et al., 2017). Since the majority of women administrators find their most significant mentoring relationship through 
informal interaction, colleges and universities should focus on creating a culture that supports the development of mentoring relationships, especially for aspiring or new administrators (Dunbar \& Kinnersley, 2011). Such a culture would include time designated for the cultivation of mentoring relationships, incentive to reward mentors for genuine time, and an expectation of mentorship embedded within the workplace environment (Dunbar \& Kinnersley, 2011).

\section{Organizational Impact}

The slow advancement of women in the upper levels of university administration continues to impasse institutions hoping to diversify their teams (Eddy \& Ward, 2015). By valuing a variety of leadership styles, including those of women, organizations can find the necessary strength and flexibility to thrive in a highly competitive and diverse economic environment (Rosener, 1990).

A lack of diversity represented in a university's leadership coincides with a lack of varying perspectives that are necessary to most effectively inform decision-making processes (Karkouti, 2016; Longman \& Madsen, 2014). Diverse perspectives have been proven to provide a relatable example for people to follow, maximize the number of alternatives in decisionmaking conversations, and strengthen utilization of the entire talent pool (Kellerman \& Rhode, 2017). Teams with a mix of men and women typically display higher levels of creativity and innovation while also engaging in more productive problem-solving tactics due to differing perspectives; traditionally, companies that have achieved gender diversity yielded strong financial results (Ellemers, 2014).

Studies have shown that with a significant representation of women on leadership teams, sales and revenue increase, industries are more productive, and reach can be much higher (Gangone \& Lennon, 2014). Allowing women to be involved in the leadership of organizations can change the values towards a system where employees work to their highest potential as a 
way of maximizing their valuable contributions to the organization; with a greater number of women in the administrative landscape, an organization's perspective enlightens to include the intentional facilitation of advancement for future women (Bilen-Green et al., 2008; Rosener, 1990). Women typically believe that pay and promotion are necessary tools of management in that employees find it meaningful to have opportunities for growth and to feel valued within the workplace for their talents and skills (Rosener, 1990). This type of culture allows employees and teams to perform more effectively when they like they are genuinely a part of the organization and can share in its success (Rosener, 1990).

Evolving ideas of gender and gender performance can help surpass individual viewpoints and help to shape the group norms and expectations in the classroom, in leadership cabinets, in residence halls, and beyond (Khwaja et al., 2017). By accepting more women into the administrative hierarchy in higher education, these women could provide insight and advocacy that is instrumental in improving the recruitment, retention, and promotion of other women employees (Bilen-Green et al., 2008). In higher education, women have been known to positively influence leadership through the following processes, among others: participatory decision making, shared and team approaches, collaboration, relational and inclusiveness orientation, harnessing multiple perspectives, empowerment, development and learning, ethics, integrity, and establishing common purpose (Kezar, 2014).

Further, there is a significant need to raise awareness for the vital benefits of gender parity and a need to educate others on the importance of achieving gender diversity in leadership roles in higher education (Teague \& Bobby, 2014). Educational policy choices could benefit greatly from the values, concerns, and issues of women, as well as from insights of feminist theory (Marshall et al., 2017). The priorities, competencies, voices, and values of women are tremendously useful when creating more inclusive, family-orientated, and collaborative 
educational environments (Marshall et al., 2017). Research suggests that institutions seeking to benefit from gender diversity should focus on identifying and mitigating barriers which block women from making the same career decisions as men. Implicit bias remains a significant factor preventing women from succeeding in men-dominated workplaces as well as inherent practices that imply that women must give up family life in order to have a successful career (Ellemers, 2014). According to McKinsey and Company (2019), a more diverse workforce will naturally build a more inclusive culture; when a culture feels fair and inclusive, women in underrepresented groups are more content and therefore, more likely to thrive.

\section{Gender and Leadership at Land-Grant Institutions}

Across institution types, women are typically concentrated in assistant and associate professor ranks and in 2008, women comprised $26.5 \%$ of tenured faculty roles and $19.7 \%$ of full professor roles at research institutions (Bilen-Green et al., 2008). Land-grant institutions had an even lower rate, with 5\% less women represented than in general public institutions (Bilen-Green et al., 2008). In 2016, 44\% of all full-time faculty members were women with only one-third being tenured or tenure-track across all institution types (Ford, 2016).

Though the number of public research institutions with women in top leadership positions is growing, the percentage of land-grant institutions with women presidents is less than half of the percentage of non land-grant institutions with women in the highest leadership position (Bilen-Green et al, 2008). "The lower incidence of female presidents in land-grants is almost predictable considering the prominence of male-dominated fields of agriculture and engineering in those schools, the fewer women presidents and provosts in the non-public universities is more unexpected" (Bilen-Green et al., 2008, p. 9). The Morrill Land-Grant Act of 1862, which created the foundation for Mid-Atlantic University and other colleges, was rooted in an intention to provide higher education access to men of the then 'industrial classes', offering formal education 
in the practical and applicable subjects of agriculture and mechanical arts (Sorber, 2013). As Bilen-Green (2008) noted, it has taken institutions of higher education, and land-grant universities, time over the past 150 years to welcome, train, hire, and promote women at institutions that were not initially created to serve them or in fields of study that they could not always easily access.

As recent as 1985 , there was still little to no research or mention of the role and status of women at land-grant institutions (Thorne, 1985). As Thorne (1985, p. 1) explained,

Like many women in other historical milieus, women at land-grant institutions have been invisible. Because they were rarely administrators, because they composed such a small part of the faculty, and often because of the discipline in which they taught, their contributions have been obscured.

Representation for women faculty members at land-grant institutions has increased since 2005; despite an increase in women faculty members over time, there is not a similar translation to women in leadership roles at land-grant institutions (Cho et al., 2017). Since the role of department chair and similar administrative leadership roles are typically filled from within the college level, it is probable that a lack of women in faculty roles could predict a similar gender imbalance at higher levels of leadership as well, especially within agriculture and science fields (Cho et al., 2017).

Though women represented only $30 \%$ of university presidencies in 2016 , more than half of current presidents are expected to step down before 2021, leaving potential opportunity for a more diverse selection of future leaders and an urgency to consider women's access to such roles (Eddy \& Ward, 2015; Gagliardi et al., 2017). According to Henneberry et al. (2002), there has been progress for women throughout various career levels at land-grant institutions, but the progress is not enough. There are growing needs to improve access and retention of women 
faculty in order to create change throughout other facets of land-grant institutions, and there is a call for further research specifically related to the retention and promotion of women at landgrant institutions (Henneberry et al., 2002).

Due to scope of these public, land-grant, research institutions, like Mid-Atlantic University, each institution's leader and leadership team must be well-equipped to manage the various facets of education, athletics, alumni, healthcare systems, and beyond. "Because athletics are inextricably tied to the growing entrepreneurialism of many research universities, the fundraising, public relations, and the partnership-building components of a university president's role can be complicated by the sometimes still male-dominant sports culture," (Hart, 2014, p. 201). Land-grant institutions specifically face a challenge to diversify the gender represented in leadership roles, especially those striving to maintain research status, honoring land-grant designation, building high profile athletic programs, and thriving academic medicine units (Hart, 2014).

As Gavazzi et al. (2018, p.160) said,

The land-grant institution of the twenty-first century must be the go-to university for state citizens across a wide range of academic, business, and technological needs. And for that to happen, there needs to be a directional alignment among many competing priorities which could never have been experienced, much less imagined, in the nineteenth century.

This process relies heavily on presidents and chancellors, and requires "intentional leadership on many fronts," (Gavazzi et al., 2018, p. 160). While there are several research studies exploring gender within specific academic disciplines including STEM, agriculture, and farming, there is a notable gap in the research related to gender and leadership in a broad context at land-grant institutions, especially related to trends in the 2000s and beyond; addressing this gap in the 
research is pertinent to coincide with ongoing calls to action for land-grant institutions to adapt their processes moving forward (Gavazzi et al., 2018).

\section{Mid-Atlantic University}

Created by the state's legislature under the Morrill Land-Grant Colleges Act of 1862, Mid-Atlantic University was founded as an agricultural college in 1867 (Sorber, 2013). Since then, the university has grown to operate several campuses statewide, a "family of distinctive campuses united by a single mission”, while maintaining primary operation in its flagship location where annual enrollment averages more than 26,000 students. With large undergraduate and graduate student populations, Mid-Atlantic University offers an extensive array of academic programs, including robust engineering and agricultural paths, medical education, and law. The university is one of only about a dozen schools in the United States that serve as land-grant institutions with a comprehensive medical school. In addition to operating as a land-grant institution, Mid-Atlantic University also maintains its status as a space-grant institution, meaning it belongs to a network of schools that are deeply engaged in research focused on outer space.

Highlighting diversity, inclusivity, the advancement of education and healthcare, and high impact research along with a dedication to honoring its land-grant status, the mission of the university focuses on advancing the school and its state into a prosperous future:

As a land-grant institution, the faculty, staff and students at MU commit to creating a diverse and inclusive culture that advances education, healthcare and prosperity for all by providing access and opportunity; by advancing high-impact research; and by leading transformation in [state name] and the world through local, state and global engagement. Staying close to its original agricultural roots, Mid-Atlantic University maintains several university farms and operates vigorous extension programming. 
In 2016, Mid-Atlantic University received Carnegie R1 (Highest Activity) Research Status designation by the Carnegie Classification of Institutions in Higher Education and has maintained it since then. Additionally, Mid-Atlantic University is among the $6 \%$ of universities recognized by the Carnegie Foundation's Community Engagement Classification. When MU earned its R1 status for Highest Research Activity, the institution affirmed its strong focus on operating top tier laboratories to advance research at the state's only land-grant and research university. In 2017, MU faculty conducted \$185 million in sponsored contracts and research grants. Mid-Atlantic University, as the largest university in the state, produces the highest economic impact statewide with $\$ 1.4$ billion in 2017 . With a return on investment of $\$ 7.50$ for each state dollar invested, Mid-Atlantic University is an impetus for growth and change in its local region. Included in this economic impact is the university's healthcare system, which is the state's largest healthcare system and its largest private employer.

In the Mid-Atlantic University system, $82 \%$ of full-time instructional faculty hold the highest academic degree in their field. MU's main campus employs 5,667 full-time and 861 parttime faculty and staff members. MU is governed by the Mid-Atlantic University Board of Governors and obtains direction regarding its public policy agenda from the Higher Education Policy Commission of its state. In 2020, the Mid-Atlantic University Board of Governors included 12 men and five women.

Throughout history, Mid-Atlantic University has only had one woman in its chief leadership position; she served as the Interim President from 1985-1986. As the first and only woman to occupy the president's office at MU, her short term was monumental. At the time of appointment, she had been serving as the Dean of the College of Human Resources and Education. Looking back on her term, she inferred that her appointment was strategic: "What happened is, the Board of Regents had a policy that if you wanted to be a candidate for the job, 
you couldn't serve as interim president. And all the vice presidents wanted to become candidates. The chancellor's office had to look for another person - one who wasn't interested in the position." She returned to her position as Dean after MU hired its next President; she went on to serve as President at two other institutions before retiring.

She was preceded by 19 men and followed by six men leading up to the university's current leadership under President Brown (name changed). In 2020, MU identified its University Leadership to include 16 women and 25 men. The roles filled by women included: Associate Vice President and Executive Director of Global Affairs, Vice President and Chief Financial Officer, Dean of Libraries, Executive Officer, Dean of the School of Nursing, Regional Campus President, Special Assistant to the Board of Governors, Vice President for University Relations and Enrollment Management, Dean of the College of Media, Dean of the College of Education and Human Services, Vice President for Diversity, Equity, and Inclusion, Provost and Vice President of Academic Affairs, President and CEO of the MU Foundation, Executive Director of University Events and Liaison to the President, and General Counsel, among others.

As explained, land-grant institutions such as Mid-Atlantic University are led by a majority of men and are not immune to the cultural challenges that can create toxic, immobilizing environments for women to succeed and lead in.

\section{Women's Initiatives at Mid-Atlantic University}

Women and men must actively seek opportunities to sponsor women as they navigate the political landscape of higher education institutions (Gangone \& Lennon, 2014). Colleges and universities that want to diversify their administration are paralyzing their own advancement by not prioritizing the progression of women in leadership roles (Eddy \& Ward, 2015). By creating a more distinct team of diverse institutional leaders, institutions can better position themselves to 
meet the challenges of an evolving field while utilizing a variety of different strategies, skills, and opinions (Eddy \& Ward, 2015). Among these strategies, initiatives for mentorship and sponsorship are critical, especially in areas where formal relationships and connections might be lacking or departments where women might not have natural or easy access to like-minded champions (Kellerman \& Rhode, 2014). Multiple coherent leadership programs for men and women are necessary, as are various forms of women's leadership programs for distinct, and in some cases, overlapping populations of students, faculty, staff, and administrators (Keohane, 2014).

These leadership development programs, especially those tailored for women, should provide opportunities for participants to explore their authentic selves while developing a capability to utilize leadership tactics associated with both feminine and masculine norms (Sulpizio, 2014). By showcasing women's leadership as an attainable process through intentional women-centered programming, participants will experience pivotal shifts in their mindsets to recognize women's capabilities to lead in both official and unofficial capacities (Sulpizio, 2014). At Mid-Atlantic University, several initiatives exist to serve various facets of women's development, leadership, and overall status within the state and university community.

\section{MU ADVANCE Center}

A notable resource at Mid-Atlantic University is the MU ADVANCE Center for research and practice in faculty and leadership development. After receiving a five-year National Science Foundation grant for $\$ 3.8$ million in 2010, the MU ADVANCE Center set out to create a more diverse workforce in science, technology, engineering, and math careers by creating intentional strategies to ensure that MU was a viable and attractive institution to women scientists. Though the primary goal of this program was to support the advancement of women in the College of Arts and Sciences and the College of Engineering and Mineral Resources, the MU ADVANCE 
Center has expanded to serve faculty throughout the university. Initiatives include supporting the academic culture, improving institutional policies, addressing the underrepresentation of women in faculty and leadership roles, understanding and supporting a stronger work-life satisfaction, promoting career support through leadership training and mentoring, and advancing research on topics affecting minority and marginalized women.

The MU ADVANCE Center seeks to provide information and resources for groups in relation to changing group dynamics and building a more inclusive organizational climate by offering a number of signature initiatives which include department dialogues, a promotion and tenure clinic, sponsorship programs, organization and training for men allies, and tools to reach group consensus. The MU ADVANCE Center also provides support to the Women's Leadership Initiative, shares information regarding the lactation network on campus, and develops customized facilitation training to achieve increased inclusivity, teambuilding, and effectiveness for groups on campus.

\section{Center for Women's and Gender Studies}

An academic unit at Mid-Atlantic University, the Center for Women's and Gender Studies provides a central location for discourse relating to the overall field of women's and gender studies and offers several degree programs. The Center's purpose includes efforts that "examine the contributions, perspectives, experiences, roles, and status of individuals within an historical and multicultural framework" and challenge stereotypes by empowering students to explore the ways gender, socioeconomic class, sexual identity, race, ethnicity, and age shape the experiences of those around us. The Center encourages students to look closely at how femininity and masculinity are socially and culturally molded rather than predetermined by biology, and to explore female behavior and the lived realities of women's lives. 
The Center annually enrolls an average of 2,000 students throughout the offered courses and engages more than 100 faculty associates, with new courses being continually added. In addition to its academic programs and pathways, the Center for Women's and Gender Studies creates opportunities for individuals to learn more about the field including fireside chats, a Scholars in Residence program, scholarships and awards, educational presentations, and special events.

In 2016, the Center for Women's and Gender Studies at Mid-Atlantic University hosted a yearlong celebration to commemorate the $125^{\text {th }}$ anniversary of women at MU; among the symposiums, lectures, exhibits, and special events, MU announced the establishment of an endowed professorship named for the first woman graduate of the university in 1891. The named Professor of Women's and Gender Studies now serves as an interdisciplinary chaired professorship intended to initiate programs, curriculum, and research to advance their own department as well as the Center for Women's and Gender Studies. As the university librarian and bibliographer for the Center for Women's and Gender Studies said,

This celebration is important because, even in the best histories of MU, women do not play a huge part in what is written. We need an ongoing history that documents not only the past and present but where we are going in the future for advancing women's opportunities and the whole picture of what women can accomplish as MU students and alumnae.

\section{Women's Leadership Initiative}

The Women's Leadership Initiative “empowers and engages women on campus, helping them achieve their full professional potential and form a community within which the participants can initiate valuable new relationships." The program launched in 2011, after a Dean attended a women's forum at Harvard University and requested a similar initiative for senior- 
level women to be created at MU under the Provost's leadership. As the program evolved, it transitioned from the Developing Women Leaders program to the Women's Leadership Initiative in which groups of women administrators were coached and later trained as coaches over the course of several years and cohorts. The program has since expanded to include forms of training, keynote speakers, and meaningful meetings with accomplished senior leaders at MidAtlantic University. Currently, the Women's Leadership Initiative provides executive leadership training for internal members of the MU community including high-level administrators, faculty, and staff; research on this training indicates increased networking, improved engagement, and an enhanced sense of community.

The Women's Leadership Initiative is led by director and a steering committee comprised of ten women whose roles include titles of President, Associate Vice President, Director, and Clinical Associate Professor, among others. In the 2018 calendar year, the Women's Leadership Initiative hosted internal events, including seminars on meditation and time management, strengths-based training, and engagements with notable keynote speakers. In 2020, the Women's Leadership Initiative engaged in networking and programming surrounding topics such as leadership in athletics, negotiation, state relations, equal pay, gender-based discrimination, and fundraising. The group does not publicly share its membership criteria and requirements; nominations are accepted annually by current members of the Women's Leadership Initiative or by current university administrators. To date, the program has trained nearly 200 women at MidAtlantic University.

\section{Women of MU}

The Women of MU "is a program created through the MU Foundation designed to provide the women who love our university another way to become engaged." This program, launched and supported by the MU Foundation, provides "alumni and friends the opportunity to 
participate in educational activities, network with other accomplished women and create a collective voice all in support of Mid-Atlantic University." With a mission to learn, experience, and celebrate, the Women of MU utilizes its position within the MU Foundation to empower and inspire women to engage in philanthropy and advocacy for the university. Each year, the Women of MU's Leadership Council selects awardees to receive funding for projects and research that directly benefit women and the university's home state.

\section{Council for Women's Concerns}

The Council for Women's Concerns was founded in 1977 to organize responses and advocacy to prominent women's issues at Mid-Atlantic University and its surrounding and connected community. Over time, the organization has grown to organize and sponsor conferences and opportunities that support women's progress. Today, the Council for Women's Concerns prioritizes the cultivation of an inclusive university community by upholding its mission to "promote equality and empowers women in all areas of the University and local community by facilitating a dialogue on women's issues and serving as a resource for women and girls."

Each year, the Council for Women's Concerns elects new members through an application process. Members then serve a three-year term and participate in leadership by fulfilling duties on one or more committees which include: Women of Color, Climate, Membership and Awards, Campus Climate, Wellbeing, and Power Based Personal Violence/Education. The Council is currently comprised of men and women who serve in faculty, staff, administrator, and student roles at MU, as well as community members. Additionally, the Council holds ex-officio seats for a representative from the Provost's Office, the Division of Diversity, Equity, and Inclusion, the Women's Resource Center, and the LGBTQ+ Resource Center. 


\section{Women's Resource Center}

A few years ago, the Council for Women's Concerns launched an initiative to build and support a center for women's resources at Mid-Atlantic University. The Women's Resource Center, though still in its early years of operation, provides an inclusive environment where students, faculty, staff, and community members are "encouraged to participate in programs, obtain resources and services, and engage in dialogue related to gender awareness and equity." The Center offers services, resources, and programs for women and people of all genders, including women's leadership workshops, resource libraries, celebrations of art and culture, campus outreach events, feminist activism and organizing workshops, and lecture series, among others. The Women's Resource Center, located currently in a temporary space, offers in-person resources as well, including free pregnancy tests, free menstrual products, a lactation room for new parents, and free contraceptives.

\section{Women Moving Forward}

Comprised of women and men from across the state and its universities, the Women Moving Forward (state named redacted from organization name) initiative seeks to confront some of the most urgent challenges unique to local women in order to secure a stronger economic future for the state. With less than half of the state's women currently in the labor force, and with one of the lowest workforce participation rates in the state, MU, its peer institutions, and its state launched this initiative to empower women and achieve long-term economic prosperity.

In 2019, the group met at Mid-Atlantic University on several occasions to utilize its over 100 volunteers to propose ideas for "disruptive, creative, and productive action that can lead to real results" related to: 
- Logistical workforce barriers that prevent women from entering or reentering the workforce.

- How to create and protect educational opportunities for girls and women across the state.

- Complex, interconnected factors in women's lives that can lead to a lifelong gender pay gap compared to male peers.

This collaborative group of volunteers consists of men and women throughout the state, with the majority being women who are employed by or are deeply connected to Mid-Atlantic University.

It is important to note that this is not an exhaustive list of all women-centered programming at Mid-Atlantic University, but rather, an overview of several programs and units serving women in the community. There are additional programs that are college-specific, including the MU College of Law Women's Leadership Council, which “works to promote an understanding of issues encountered by women in the community and in the law school environment." This group strives to encourage women to redefine their role in the legal environment and profession, while also encouraging women to excel in law school and develop a broad awareness about the legal, social, and economic issues faced by women and all people in the state.

\section{Conclusion}

"What appear to be gender neutral rules applicable to everyone are actually expressions of the gendered university - a place in which the terms of hire, tenure and promotion, salary negotiation, and advancement to leadership positions are based on an anachronistic male breadwinner model. Women are systematically disadvantaged in this male-normed environment because they present a different lived experience." (Ford, 2016, p. 500). Rooted in the environment of Mid-Atlantic University, this study explores the lived experiences of women 
leaders in men-normed environments at a land-grant institution, seeking to understand the barriers they face and overcome in this institutional context, in order to lead, aspire to lead, and impact among their institutional environments.

Current research does not address the pathways for women to become high-level leaders at land-grant institutions nor the lived experiences of the few women currently holding those positions; this study identifies pathways and attrition points while discovering strategies and skills employed by women leaders to attain and succeed in such roles. In doing so, this study illuminates the convergent and divergent experiences of unique and diverse women who work in the same institutional context. 
Chapter Three: Methodology 
Identifying the specific supports and barriers women face throughout their careers that might enable or impede them from accessing and succeeding in high-level roles in university administration, this study focuses on the reflective and projective perspectives of women leaders at a public, land-grant research institution. Specifically, this study examines the following research questions:

1. What are the attitudes, behaviors, and/or tactics that women in senior-level administrative roles employ to successfully attain a high-level position?

2. What are the policy, structural, cultural, and behavioral influences that preclude women from advancing to leadership positions at a land-grant institution?

3. What are the attrition points in the career pipeline identified by women?

\section{Overview of Methodology}

Conducted primarily by talking with people, interview research relies on gathering people's stories and accounts, learning of their experiences and perspectives, and providing those experiences a voice in academic or public discourse (DeVault \& Gross, 2012). Though interviews can often be simplified as basic human interactions, the focus on narrative inquiry rooted in feminist and role congruity theories elevates this research to a purpose of better understanding by informing the processes to which women leaders access and operate in highlevel leadership roles at land-grant institutions. This simplification of interviews to fundamental human interactions neglects the complex powers of language, interaction, translation, listening, dynamics of power, recall patterns, and interpretation (DeVault \& Gross, 2012). With this in mind, the researcher utilized strategic methods to uphold feminist research focused broadly on telling the stories of women leaders through narrative inquiry.

\section{Feminist Narrative Inquiry}


"Qualitative research must be conducted with an understanding of how the broader social order oppresses different categories of people by race, gender, or class," (Taylor et al., 2015, p. 25). Seeking to understand the complexities of women's lives while promoting social justice and social change, feminist research is often posed toward the goal of accessing and exploring the voices of those who are marginalized and, in this case, those of women (Hesse-Biber \& Leavy, 2007). Feminism can exist as both theory and practice, and in research, the feminist perspective assumes a political commitment by researchers to produce useful knowledge that might make a difference in the lives of women (Letherby, 2003). Feminist theory and research lean on the principle that conditions for women must be changed for the better (Kohli \& Burbules, 2012). Feminist research then can be distinguished by the ways in which questions are asked, the frames through which perspectives are analyzed, and the overall purpose of the study (Letherby, 2003).

Historically, women and women's concerns have not received a great deal of attention from researchers and when they have, research has often left women at the center of studies feeling objectified and further 'othered' (Letherby, 2003). With this in mind, feminist researchers must acknowledge the messiness of the research process and approach the process with detail, intention, and extreme sensitivity while producing new knowledge on the subject (Letherby, 2003). In many ways, feminist ethnographers should use reflective tactics to mitigate and diminish how domination and oppression are replicated in the course of their research (Naples, 2003). It is common for feminist-focused researchers to exercise reflexivity throughout the interview process, keeping the research accountable of their own personal positionality and that of the informant (Hesse-Biber \& Leavy, 2007). For researchers, the recognition should begin before entering the interview environment by questioning and exploring how one's social, political, and economic context, belief system, biography, and overall construction of knowledge might inform assumptions that interfere with the research process (Hesse-Biber \& Leavy, 2007). 
The reflective practices employed by researchers are influenced by what they understand and conceptualize as a standpoint, and how they assess and acknowledge their positionality in the field (Naples, 2003). Such reflective practices might include the presentation of the research, the nature of the ask, and the presentation of self through behavior and dress during the research process (Naples, 2003).

However, it is also essential to recognize that it is impossible to eliminate all power imbalances (Naples, 2003). "Feminist researchers are neither immune to the internalized oppression nor the hegemonic constructions of research practice that insist on a distanced and objectifying angle of vision" (Naples, 2003, p. 197). With this in mind, the researcher utilized reflexive and reflective processes to conceptualize her standpoint and acknowledge the positionality with which it presents in the context of her research. In practice, this involved sharing her motivation for pursuing the research topic with each informant while developing rapport, journaling throughout the data collection process, and revisiting participant interview recordings to confirm understanding and memory of tone and demeanor.

Narrative inquiry includes several approaches that rely on written or spoken words to tell a story, with narrative analysis then focused on incorporating the accounts in first person to emphasize an individual's experience (Lichtman, 2014). In its simplest form, narrative inquiry and analysis uses the told and lived stories of people to bring meaning to the experiences related to their account, which may connect to those of others in the process of research (Lichtman, 2014). Intrigued by how people narrate their own versions of reality, researchers utilize narrative inquiry to understand the structural aspects of people's stories hoping to understand how they make sense of their lives (Taylor et al., 2015).

While other methods might seek to understand research participants over time or through the lens of emerging theory, narrative inquiry focuses on the study of life through individual 
stories, which are illuminated in the interview process and can be used to connect themes and perspectives of individuals with similar experiences and stories (Lichtman, 2014). Narrative inquiry and stories lived and told can create spaces and gaps that point towards a direction of change: change in researchers, changes in participants, change in the institutional landscapes, and change in the potential readers of the narrative inquiry (Pushor \& Clandinin, 2009).

This feminist-based narrative inquiry loosely relied on concepts of institutional ethnography, which focuses on the experiences of individuals whose everyday activities are linked to and impacted by institutional relations, reiterating the importance and exploration of research that explores these environments (DeVault \& McCoy, 2002). Smith (2005) positioned institutional ethnography as a component of alternative sociology in which researchers seek to understand informants' perspectives in hopes of better identifying the relations of ruling that shape local experiences. More simply, institutional ethnography, through various methods, investigates the linkages between settings of everyday life and organizations, as well as local administration and governance processes (Smith, 2005).

"When interviews are used in this approach, they are used not to reveal subjective states, but to locate and trace points of connection among individuals working in different parts of institutional complexes of activity" (DeVault \& McCoy, 2002, p. 371). Through identifying how the relations of ruling render invisible gender dynamics embedded into bureaucratic structures, a feminist approach can reveal how inequality is produced and reproduced in everyday life (Naples, 2003). Institutional ethnography relies heavily on interviewing, observation, and documents to peek into a window of informants' perspectives to conceptually frame and further explore how individuals' lives are inextricably linked to institutional environment and action (Campbell, 1998). While this study focuses explicitly on narrative inquiry, there are subthemes 
of institutional ethnography woven throughout the research and reporting, creating a fuller picture of the experiences and context related to the research itself.

\section{Conceptual Framework}

The researcher utilized feminist-based research frameworks and role congruity theory to preserve a strong focus on the advancement of women and the effective use of the research participants' storytelling to inform findings that contribute to the gap in research regarding women leaders at land-grant institutions.

\section{Feminist Theory}

Applying feminist theory and tenants of third-wave feminism, such as intersectionality and patriarchal impact on organizations, to deconstruct the dominant discourse on gender-based leadership can widen the complex understandings of social life and construct new visions within educational systems that are focused on creating community and collaboration for diverse groups of people and leaders (Marshal et al., 2017). In this sense, feminist perspectives can also contribute to the elimination of patriarchal systems and the dissolution of societal assumptions that women are meant to be controlled (Marshal et al., 2017).

Throughout the study, the researcher maintained attention to the intersecting identities of the women informants and exerted effort to ensure that participation in this study did not create additional othering for those willing to share their stories to advance the research.

\section{Role Congruity Theory}

The perceived incongruity that exists between a woman's gender role and their leadership role ultimately initiates two specific forms of prejudice: the perception of women as less favorable occupants of leadership roles compared to the perceptions of men and the evaluation of behavior that leader roles are viewed less favorably when held by a woman (Eagly \& Karau, 2002). "According to the role congruity theory of prejudice, the application of the female 
stereotype to women, with its lower level of agency, should decrease a woman's chances of being perceived as possessing agentic attributes” (Eagly \& Karau, 2002, p. 583).

The role congruity theory reiterates the notion that women in leadership roles are stereotypically judged based on predisposed ideas and prejudiced attributes of nurturing, mothering characteristics; this theory asserts that the influence of gender roles on behavior limits women's success in accessing and thriving in leadership roles (Eagly \& Karau, 2002). Through this lens, women are less likely than men to emerge as leaders when expectations for the role are incongruent with gender-based stereotypes (Paustian-Underdahl et al., 2014; Ritter \& Yoder, 2004). In fact, the combination of masculine leader association and masculine task association will predict that women are less likely to emerge as leaders under such conditions, allowing the man as the dominant leader to emerge (Ritter \& Yoder, 2004). Due to this phenomenon, attitudes are typically less positive toward current women leaders and dismissive of the potential for women to be leaders compared to those of men leaders (Eagly \& Karau, 2002). These attitudes and perceptions, among other factors, create an environment that makes it more difficult for women to become leaders and achieve success in leadership roles (Eagly \& Karau, 2002).

Utilizing this theory allows the researcher to identify the distinct prejudiced perceptions of women in leadership roles while contextually framing expectations of women based on a measure of masculinity. Additionally, this theory provides a foundational reference point for perceptions of feminine leadership to be those of minimal qualification, nurturing leadership styles, and a general deficiency in effectiveness. In the context of this study, feminist theory and role congruity inform the researcher's methods to advance the knowledge related to women's advancement in higher education leadership roles while maintaining a strong focus on creating and sharing literature to decrease the negative influence of gender roles on women's career trajectories. 


\section{Data Collection}

By conducting personal in-depth interviews, researchers can gain insight into understanding the subjects' lives, experiences, and situations as expressed through their own narratives (Taylor et al., 2015). When interviewing multiple informants, researchers can utilize analytic induction to build general theories about a social phenomenon or shared experience (Taylor et al., 2015). In this study, the researcher engaged in feminist-based narrative inquiry through personal interviews to gain a deeper understanding of the trajectory each woman followed to arrive at her current role, barriers she overcame throughout her career, challenges she might have faced and currently faces, attrition points she encountered, and insight she might have regarding how to create a smoother path for women moving forward.

\section{Research Site and Setting}

To best maximize opportunity for developing theoretical perspectives based on linkage and connection, it is recommended to conduct studies in multiple settings (Taylor et al., 2015); in this case, research was conducted at one institution but in 18 different units, sectors, and disciplines. Naturally, this allowed for the researcher to identify correlation and common themes among the participants' narratives that shed light on patterns of success and areas of growth related to women's advancement at Mid-Atlantic University. Though universities operate as one cohesive unit, there are multiple perspectives to be gained and understood by gathering data from a variety of functional areas and academic units within a large institution.

For the purpose of this study, research was conducted at various levels of administrative leadership roles held by women leaders at a public, land-grant, research-focused institution located in the mid-atlantic region of the United States: Mid-Atlantic University. This institution, characterized by a pseudonym throughout the report, was chosen based on key factors including the representation of women in the university's leadership and the intention to illuminate the 
experiences of women at this and similar institutions. The researcher completed the necessary protocols to gain access to and ethically conduct research in this setting, including approval from the Institutional Review Board of West Virginia University, where the researcher was enrolled.

When conducting qualitative interviews, it is imperative for researchers to construct an atmosphere in which there can be an open discussion in a relaxed, natural setting (Taylor et al., 2015). Knowing that informants would likely be hesitant to discuss emotional, sensitive, or potentially confidential topics in an unfamiliar or unsafe space, researchers should be flexible in providing choice or offering alternative settings (Letherby, 2003). In this study, informants were to be offered an opportunity to choose the site and setting for their research, whether that might be their home, their office, or another type of private, comfortable setting. However, due to the unforeseen impact of the global pandemic, COVID-19, and the related order for individuals to stay at home during the timeframe of data collection, all interviews were conducted using the secure Zoom video platform. This platform was provided free of charge, with a secure login, to all faculty and staff of the university which at the time, included all study participants.

\section{Sample}

For the purpose of this study, the researcher targeted a sample of women in academic and non-academic administrative leadership positions at Mid-Atlantic University. With a focus on gaining an in-depth understanding of the process and meaning that individuals attribute to their situation, it is not uncommon for qualitative interviewing to involve a small sample size (HesseBiber \& Leavy, 2007). To better understand and explore the experiences of the women leaders in this study, the researcher sought a purposive small sample of 15 to 20 women and resulted with 18 participants.

\section{Selection and Recruitment}


Women were recruited to participate in this study who were currently serving at levels of identified leadership including or equivalent to: Vice President, Associate Vice President, Assistant Vice President, Provost, Vice Provost, Associate Provost, Dean, Associate Dean, General Counsel, and Executive Director. This criteria was designed to intentionally seek perspective regarding each woman's career trajectory, the challenges she has overcome to reach her position, barriers she may or may not still face, and insight she has regarding the process for future women to attain and succeed in administrative leadership roles at Mid-Atlantic University and similar institutions.

As there is no clearly identified path for individuals to prepare for and attain administrative leadership roles in higher education, the researcher focused on women in both academic and non-academic positions, recognizing the potential for women to have held a number of academic and non-academic positions throughout their career that may or may not be linked to the knowledge and scope of the current role. Focusing on either academic or nonacademic positions would have limited critical perspectives regarding one's career trajectory and to avoid this limitation, the researcher strategically recruited participants in both academic and non-academic roles.

As we know, the method of qualitative interviewing requires flexible research design, allowing for the number of informants and the nature of the interview to evolve throughout the process, including after the initial interviews have been conducted (Taylor et al., 2015). Informants received an invitation to participate in this study by personal email from the researcher directly, which can be reviewed in Appendix A along with the letter of informed consent, which was included in the message to prospective participants.

Institutions of higher education are gendered, raced, and classed sites to live, work, and learn; the work of faculty, staff, and administrators involves positions at these intersections 
(Maguire \& Berge, 2009). In an ideal research setting, the observer is able to obtain easy access, develop an immediate rapport with the observant, and solicit or gather data closely related to the research goals, a process that typically involved negotiating access, winning trust, and collecting any relevant data (Taylor et al., 2015).

In order to choose a unique set of women whose voices would predictably highlight diverse experiences from various career pathways and functional areas, the researcher first created a spreadsheet of individuals at levels of administrative leadership between the Director and President distinction in all units at the university who identified as women. From that list, the researcher then recruited one woman from each unit or functional area and continued to reach out to other women on the list until 18 participants had confirmed. It is important to note that in some university units, there were no women listed whose title matched the criteria above.

\section{Confidentiality}

In qualitative research, maintaining confidentiality typically refers to the process by which the researcher keeps hidden any identifying information regarding the study participants (Saunders et al., 2015). In this study, the major identifying information of participants was kept in confidentiality by the researcher. At a primary level, qualitative researchers should secure informed consent from interview participants, conduct interviews with methods central to exercising sensitivity for participants' feelings and concerns, and protect the identity of interviewees by changing identifying details in reports or by utilizing pseudonyms (DeVault \& Gross, 2012). At the significant risk for the women included in this sample, research informants were coded using pseudonyms to protect their identities and avoid potential for harassment, legal challenges, self-aggrandizement, and overall discretion (Taylor et al., 2015). To administer additional protection, the pseudonyms were not linked with the unit or college where the participants currently work or any other major identifiers. Former job titles and prior institutions 
of employment were omitted or edited to remove any qualifying data. After the interviews were completed, the researcher masked the study's site institution with a pseudonym, Mid-Atlantic University (MU), to provide a supplementary layer of protection based on the nature of data presented during the collection process.

\section{Interviews}

Data was collected primarily through personal interviews with the participants in this study.

\section{Semi-structured Interviews}

Semi-structured interviews, conducted with some type of interview guide, feature a number of set questions with moderate flexibility to ask follow-up questions or seek additional connection and clarification throughout the conversation (Hesse-Biber \& Leavy, 2007). In this study, the interviews were scheduled initially for 60 minutes; depending on the availability of the informants and the nature of the interview, the interviews were abbreviated or extended. The minimum interview time was 00:31:58 and the maximum was 1:05:10, with an average interview time of 00:48:39. Though the researcher planned to conduct one in-person interview with each participant, there was a required pivot to virtual video conference interviews in light of COVID19; all interviews were then scheduled through the Zoom video platform instead. The stay-athome order and unexpected impact of COVID-19 on job responsibilities, childcare, working environment, and mental health, among other factors, affected the availability of participants and in many cases, required them to shorten interviews that had been originally scheduled for 60 minutes. Additional details regarding this context are addressed in Chapter Five.

At the beginning of each interview, the researcher reviewed the technical aspects of the Zoom platform as well as the interview format and outline before answering preliminary questions. The researcher utilized methods of expressing affirmation, including nodding and 
smiling, as well as probing throughout the interview process to reinforce comprehension and the open opportunity to continue sharing in a comfortable online space.

The researcher used silent probing, echo probing, and probing by leading the informant, leaning on the interview guide to redirect if necessary (Hesse-Biber \& Leavy, 2007). In almost every case, the conversations flowed very naturally, with positive energy from the researcher and the informant which created a comfortable and trusting environment. Though the researcher utilized the interview guide to integrate each question into the conversation, more than 15 of the interviews flowed organically enough to cover each question in a natural and authentic conversation.

\section{Interview Guide}

In this study, an interview guide was utilized to organize, structure, and lead the researcher's creation of a comfortable, intentional, and focused conversation. Typically, interview guides serve as a loose outline of pre-research, intended topics, and sample questions in a format that can be adapted and expanded as the interviews occur (Hesse-Biber \& Leavy, 2007; Taylor et al., 2015).

The interview guide, categorized by relevance to each research question, included notes to lead the introduction and consent to be recorded followed by a series of questions related to the subject's background, career trajectory, career influences, challenges and barriers, working environments, service-related expectations, and land-grant philosophy. The interview guide was edited and adapted with each interview as themes, perspectives, and additional questions came to mind that helped the researcher to better understand the lived experiences of each woman while maintaining consistency and fairness throughout the process. Due to the personal nature of each interview and the uniqueness of each participant, these follow-up questions and their answers naturally varied. After the first three interviews, the researcher recognized that some questions 
were eliciting similar, if not duplicative responses from participants and causing time constraints in the interview. The researcher then eliminated two questions which allowed for a more streamlined and focused flow of questions and conversation. The final interview guide can be accessed in Appendix C.

\section{Recording}

Interviews in this study were documented using the Zoom platform's audio-only recording feature, with consent to record provided by each participant at the beginning of the interview. The conversations were also recorded through a backup audio recording device to moderate risk of technological failure. Though the idea of recording can alter the depth and trust associated with the participant's willingness to share, audio recordings allowed the interviewer to capture more than she could from memory and revisit the conversation to identify additional factors including tone, hesitation, and previously unnoted themes (Taylor et al., 2015). The recordings were saved through West Virginia University's Remote VPN on the Zoom platform, using the researcher's student login credentials.

\section{Journaling}

Journaling in the qualitative process can allow the researcher to note emerging themes, observe gestures and nonverbal expressions, and gain insight regarding patterns among participants (Taylor et al., 2015). The researcher utilized ongoing and post-interview journaling to synthesize interview conversations and experiences. The researcher also reflected upon interactions before and after the interview recording, feelings experienced, nonverbal communication and body language noted during the interviews, and notes regarding any additional communication with the research informants.

\section{Data Analysis}


Participant interview transcripts were the main source of information in this study, combined with contextual information regarding the research setting and organizational culture. The researcher utilized a three-step process to analyze interview data, including data reduction, data reorganization, and data representation (Roulston, 2014). Once the data collection was complete, the researcher removed names from the audio recordings. The recordings were then transcribed using a professional and secure transcription service, Landmark Associates, that captured strict verbatim responses and non-verbal sounds. The transcripts were then transferred to the NVivo software, password protected through the researcher's West Virginia University Remote VPN, for coding and analysis.

\section{Coding}

The researcher coded the interview transcripts based on emerging themes, similarities, and prevailing patterns. The coding process, loosely rooted in Lichtman's (2014) general coding tactics, categories, and constructs, was operated by attributing codes to items where these themes, similarities, and patterns occur, then used to organize and categorize items accordingly. As Lichtman (2014) recommended, codes should be continually refined, added, and eliminated to maintain an iterative process.

First, the researcher labeled each transcript with the appropriate pseudonym and removed any institution or personal names from the participant narratives for confidentiality purposes. Then, the researcher assigned case labels, designating whether each participant currently held an academic or non-academic leadership position at Mid-Atlantic University at the time of the interview in June 2020. Repeating this process, the researcher also labeled each case with the participants' age range and confirmation regarding whether or not they had experience working professionally outside of Mid-Atlantic University and outside of higher education. Table 1 shows the cases assigned to participant transcripts: 


\section{Table 1}

\section{Case Overview}

\begin{tabular}{ll}
\hline \multicolumn{1}{c}{ Case } & \multicolumn{1}{c}{ Case Classification } \\
\hline Age & (numerical data) \\
Type of role & Academic; non-academic \\
Worked outside of MU & Yes; no \\
Worked outside of higher education & Yes; no \\
\hline
\end{tabular}

During the first round of coding, the researcher assigned codes based on the specific question group being answered. Once this process was complete, and the researcher had identified several themes among each question group, the researcher coded the data a second time with broader codes and subcodes added to the initial question group codes. After all transcripts had been coded with this set of new codes, the researcher reviewed the data a third time, continuing to assign codes to the participants' responses to ensure that all ideas were adequately represented by the question group codes, broad codes, and subcodes. No codes or subcodes were added in this third round of coding. Table 2 identifies the question group codes and broad codes, as well as subcodes by which the data were coded and analyzed. 
Table 2

Code Overview

\begin{tabular}{ll}
\hline \multicolumn{1}{c}{ Codes } & \multicolumn{1}{c}{ Subcodes } \\
\hline Q1 Career Path & $\begin{array}{l}\text { Faculty; unplanned trajectory; headhunted, persistence; } \\
\text { promotion by supervisor }\end{array}$ \\
Q2 Positive Influences & $\begin{array}{l}\text { Female friend; helping students; leadership program; } \\
\text { mentorship; positive role model; negative role model; } \\
\text { network; supervisor }\end{array}$ \\
& $\begin{array}{l}\text { Gaining respect; job-related; organizational politics; } \\
\text { racism }\end{array}$ \\
Q3 Barriers and Challenges & Consulting; level up; retirement \\
& Confidence; gain knowledge; softness \\
Q4 What's Next & Committees; confirmation of burden; minority groups; \\
Q5 Tactics Employed & note-taking \\
Q6 Service Burden & Appearance; gender rules; hiring and recruiting; \\
& unequal pay; negotiation; that moment; reporting; \\
Q7 Gender-Based Discrimination & women vs. women \\
& Connected; not connected \\
Q8 Land Grant Mission & Emotional regulation; find mentors; find your people; \\
know your purpose; learn and study; network, say yes; & seek and create opportunities; volunteer \\
& Create change; open doors for others; role model \\
Q10 Meaning of Role & Women of color; LGBTQ+ \\
Underrepresented Identities & Positive progress; provost; room for growth \\
MU Environment & Husband; children \\
Unprompted Family & \\
Highlight Quotes & \\
Literature Recommendations &
\end{tabular}

After assigning these labels to the data, the researcher then explored each individual coded set of data to reflect, memo, and analyze patterns that emerged from the participants' narratives. From there, the researcher was able to thematically group broad ideas into the implications addressed in Chapter 5.

\section{Trustworthiness of Research}

Quality research involves methods for direct investigation and yields clear chains of reasoning based on experimental or quasi-experimental findings, producing generalizations that 
can be replicated and utilized to test theory (National Research Council [NRC], 2005). In an effort to conduct research that can be applied and replicated, the researcher focused on ethics and transferability throughout the study. The qualitative researcher's perspective has the potential to influence the collection of evidence in ways that might solicit a lack of trust in the research process, undermining the credibility and warrantability of the findings (Denzin, 2009). To avoid this occurrence, researchers should increase transparency by discussing processes of interpretation and analysis, highlighting alternative interpretations, and providing contextual commentary, along with clearly indicating the sampling frame and the domains to which any generalizations might apply (Denzin, 2009).

Lincoln and Guba (1985, p. 290) provided a helpful framework for evaluating the trustworthiness and worth of research studies by asking four key questions:

- Truth value: How can researchers establish confidence in the truth of the findings of an inquiry for the subjects and with the context in which the inquiry was carried out?

- Applicability: How can researchers determine the extent to which the findings of an inquiry have applicability to other contexts or related to other respondents?

- Consistency: How can researchers determine whether the findings of an inquiry would be replicated if the inquiry were duplicated with similar respondents and in a similar context?

- Neutrality: How can researchers establish the degree to which the findings of an inquiry are determined by the respondents and the conditions of the inquiry instead of by biases, motivations, interests, and perspectives of the researcher?

\section{Credibility and Confirmability}

Referring to the extent to which a research account is appropriate and believable, credibility is a key factor in determining the extent to which research is trustworthy and should 
be valued alongside similar criteria for determining reliability, plausibility, validity, authenticity, and generalizability (McGinn, 2012). To establish credibility and confirmability, Lincoln and Guba (1985) recommended prolonged engagement, triangulation, referential adequacy, and member-checking, among other strategies. This study utilized a combination of these tactics to reinforce credibility, including triangulation and reflexivity.

By conducting this study in the context of a public, research-focused, land-grant institution, comparing the experiences of women leaders in this environment while considering current events and gender-based factors within the organization, the researcher utilized triangulation to strengthen credibility. In this context, triangulation is rooted in the search for convergence between multiple perspectives, allowing for a broad cross-check of data and utilization of several perspectives to reinforce themes and interpretation (Krefting, 1991).

In addition, the researcher operated a reflexive approach, documenting and acknowledging her own biases and perspectives that might influence the data collection and interpretation processes, as mentioned above (McGinn, 2012). This reflexive approach allows the researcher to appreciate the value of each interview conversation, recognizing that it is no simple encounter but rather, is shaped by the cultural constructions of similarity and differences (DeVault \& Gross, 2012). In certain contexts, this involved the researcher's open sharing of research interests and political commitments in a way that might allow the conversation to hold a collaborative moment of making knowledge (DeVault \& Gross, 2012). By operating reflexively, the researcher can consider her own biography in the context of the research relationships, interviews, and analysis, understanding how her context informs her perspective (DeVault \& Gross, 2012).

\section{Transferability}


In this study, methods to reinforce transferability are especially imperative as the researcher will be seeking to make generalizations across the experiences and perspectives of research subjects (Krefting, 1991). To reinforce transferability, the researcher offers a concentrated description in the following chapter with sufficient detail of the full interview experience, making explicit the patterns and social relationships to allow for a deeper understanding by readers (Lincoln \& Guba, 1985). This description includes key components regarding the respondents' history, general career path, and the institutional context, allowing readers to access the transferability of the findings through a broad perspective (Krefting, 1991).

\section{Dependability}

Lincoln and Guba (1985) recommended an external audit completed by a researcher outside of the research process to evaluate accuracy of the process and its findings. "Data analysis is rife with ethical issues because it exposes power and privilege in relationships, decision making around maintaining or curbing relationships with research subjects, and the potential for profound and varied relational violations," (Doucet \& Mauthner, 2012, p. 134). To ensure that ethical considerations are applied properly, researchers should seek additional review of the robust processes, taking into account any level of risk for the individuals involved and to discuss the potential challenges of each research question and its direction (Lombardo, 2017).

In this study, an extensive peer review was completed by the researcher's advisor with the intention of confirming accuracy and validity of the overall research study. This peer review involved a preliminary investigation of the research proposal, including the interview guide and research questions, as well an in-depth inquiry of the findings and conclusions.

\section{Ethics}

The ethics of social justice and the ethics of care have dominated the rationale of feminist researchers for years, seeking to expose inequalities and discredit claims that women are inferior 
to men (Preissle \& Han, 2012). "Ethical research practice must attend to the close connection between both the context of discovery and the context of justification by attending to the continuous, fluid and complex relationships that constitute qualitative research projects throughout the varied contexts and processes of knowledge construction" (Doucet \& Mauthner, 2012, p. 135).

"Women studying women, about women or with women, for the purpose of relieving women's oppression and reconfiguring androcentric knowledge into a more inclusive understanding of 'huwomanity,' complicates the research process” (Preissle \& Han, 2012, p. 527). Despite a strong focus on ensuring ethics of care, a feminist philosophical perspective that focuses on morality and context-bound decision making, ethical dilemmas are not eliminated and researchers then must ensure that decisions are rooted in utmost care and findings are communicated in methods that will not create further distress or harm (Preissle \& Han, 2012). According to the Nebraska Sociological Feminist Collective (1988), ethics in feminist research spans beyond the basic research substance to involve specific questions asked, intentions behind the study, representation of the findings, and faithfulness to the subjects and cause. As defined by the Nebraska Sociological Feminist Collective (1988, p. 535), "A feminist ethic orders these general issues in a specific perspective that recognizes women's continued oppression within a patriarchal social system and discipline. A feminist ethic identifies this continued oppression as a major contradiction of our research.”

Literature suggests that feminist researchers should, "give continuous and reflexive attention to the significance of gender as an aspect of all social life and within research, and consider further the significance of other differences between women and the relevance of men's live to a feminist understanding of the world," (Letherby, 2003, p. 73). In addition, feminist researchers should challenge the norm of knowledge as objective, develop non-exploitative 
relationships through the research process, prioritize privacy, and value reflexivity and emotion as insightful, essential parts of research (Letherby, 2003).

Though reflexivity is an integral part of the research and knowledge-seeking process, it can carry with it a heavy weight of ethical dilemma for which researchers must pay special attention to as they construct knowledge and demonstrate an ongoing commitment to soliciting and sharing the stories of women (Doucet \& Mauthner, 2012). Prior to conducting interviews, feminist researchers should critically examine the purpose of the interview and learn as much as possible about the informants before the entering the research space in order to adequately exhibit respect for the informant and in this case, her time, effort, and potential to relive painful or triggering moments related to her life and career (DeVault \& Gross, 2012). The researcher engaged in reflexivity throughout this study, as recommended by the literature, and prior to each interview, completed adequate research regarding each research participant.

\section{Conclusion}

Focused primarily on addressing the gaps in the current literature regarding the career pathways for women administrators in higher education leadership, specifically the barriers, attrition points, and lived experiences, this study utilized feminist-informed narrative design through in-person interviews to reinforce current data and produce new knowledge related to the advancement of women and universities in this context. The following chapter will discuss the findings from the series of participant interviews. 
Chapter Four: Findings 
To explore the participants' career paths and longitudinal experiences, the researcher conducted a series of interviews with 18 women in university leadership positions between the levels of Director and President at Mid-Atlantic University's Main Campus. The stories told by these women illuminated positive influences on their career trajectories while confirming the ongoing challenge of gender-based discrimination in their higher education workplaces and identifying areas for organizations to expand opportunities for women in leadership roles. In addition, the data confirmed existing research related to the unequal burden of service, ongoing disparities in responsibility, pay and expectation, and the significance of effective support systems and mentors. Stories of gender-based discrimination, memories of positive role models, tactics employed by women to navigate their working environment, and advice for aspiring leaders transpired from the data, among other patterns and themes. This chapter will provide an overview of the study participants, the stories they have shared, and the connections among them.

\section{Participant Overview}

The researcher recruited participants for this study based on their job title and position of university leadership at the time of the data collection in June 2020. During this time, eight participants held positions that the researcher categorized as 'academic', referring to leadership of or within an academic unit related to the instruction of students, oversight of faculty and department chairs, or related functions. There were 10 participants whose positions were categorized as 'non-academic', referring to job responsibilities in non-academic functions such as oversight of units that support various facets of the experience, safety, and tools for students, faculty, and staff. To protect participants' identities, many of whom are the only women in their unit, the departments and job titles have been omitted from this dissertation. 
Among the 18 study participants, there was a broad range of age and experience type.

The women's ages ranged from 39 years old to 69 years old at the time of data collection. While 12 participants have worked professionally in roles outside of higher education, six participants built their careers exclusively within the college and university setting. To protect the identities of study participants, the researcher utilized pseudonyms and age ranges. Participants are primarily referenced throughout this chapter by a pseudonym; in cases when the presentation of information reveals identifiers, the nondescript term 'participant' is applied instead to protect confidentiality. Table 3 provides an overview of the participants' names, ages, type of leadership role, and documentation of experience outside of higher education.

\section{Table 3}

Participant Overview

\begin{tabular}{|c|c|c|c|c|}
\hline Name & Age Range & $\begin{array}{l}\text { Category of } \\
\text { Current Role }\end{array}$ & $\begin{array}{l}\text { Professional } \\
\text { Experience } \\
\text { Outside of MU }\end{array}$ & $\begin{array}{l}\text { Professional } \\
\text { Experience Outside } \\
\text { of Higher Education }\end{array}$ \\
\hline Alice & $65-75$ & Academic & Yes & Yes \\
\hline Angelina & $35-45$ & Non-Academic & Yes & Yes \\
\hline Cassandra & $35-45$ & Academic & Yes & Yes \\
\hline Eleanor & $45-55$ & Academic & Yes & No \\
\hline Emma & $45-55$ & Academic & Yes & No \\
\hline Ginny & $55-65$ & Non-Academic & Yes & Yes \\
\hline Helena & $65-75$ & Academic & Yes & No \\
\hline Lily & $35-45$ & Non-Academic & Yes & Yes \\
\hline Marge & $55-65$ & Non-Academic & Yes & Yes \\
\hline Marietta & $45-55$ & Academic & Yes & No \\
\hline Miriam & $45-55$ & Non-Academic & Yes & Yes \\
\hline Molly & $35-45$ & Non-Academic & Yes & Yes \\
\hline Penelope & $35-45$ & Non-Academic & Yes & Yes \\
\hline Rita & $55-65$ & Academic & No & No \\
\hline Rose & $45-55$ & Non-Academic & Yes & Yes \\
\hline Susan & $55-65$ & Non-Academic & Yes & Yes \\
\hline Sybil & $55-65$ & Academic & Yes & No \\
\hline Veronica & $45-55$ & Non-Academic & Yes & Yes \\
\hline
\end{tabular}




\section{Career Paths}

The participants' educational and career paths evolved in a non-linear fashion, and for many, the trajectory was as they called it, "unplanned" or "nontraditional". As Cassandra said, 'Not everything was planned... Instead of a direct point A to point B, it wasn't linear, let's say. It was more like a cryptic doodle." Several women, particularly those who now hold nonacademic leadership roles, expressed belief that their path was nontraditional for this field, inferring that there is indeed a traditional path for higher education leadership positions. However, the stories of women in this study reinforced the notion that there is no straight path or clear trajectory to any of the positions they currently hold today. Simultaneously, they fortified a patriarchal narrative in that there is indeed a clear path for men.

There was a series of career opportunities and life experiences that participants believed ultimately led them to their current roles. For one participant, this involved stepping away from higher education for several years to focus on building a new business, starting her own nonprofit organization, and taking time to care for her family before returning to higher education leadership with enhanced skills and a renewed sense of purpose. It was important for some women to stay in one environment, serving students and advancing through academic leadership positions at the university level. In a few cases, women reported opportunities to write their own job descriptions and in other cases, women were asked to step down as a result of ongoing gender bias and sexism. For several women in this study, the call to pursue a career in higher education came while they were working externally as attorneys, healthcare professionals, or consultants. According to one participant, the career path and its direction have been guided entirely by faith, “When I left high school, —I'm very faith-driven—it was my prayer, 'Lord, just give me an opportunity to help people'... (after college) My prayer was still the same. Your position may change, but the prayer is still the same." 
While these women's career paths and life experiences are uniquely their own, their stories reveal common themes and confirmation that being a woman in the workplace of higher education is an ongoing challenge. The following section will explore in-depth the themes that emerged from their stories, identifying common threads throughout their career paths and experiences as outnumbered women leaders at Mid-Atlantic University.

\section{Theme 1 - Attrition Points and Positive Influences}

As participants explored their career paths in the interviews, they identified many career moments and life events that propelled their careers forward while also highlighting positive personal influences that supported their career and life journeys.

\section{Impetuses for Advancement}

The participants attributed advances in their career paths to the impact of a deliberate supervisor, professional recruitment, and personal persistence.

\section{The Influence of a Strong Supervisor}

Consistently, the participants reiterated the power of having a positive relationship with their current and previous supervisors, something that frequently led to opportunities for personal growth as well as to career advancement. They described scenarios when their supervisor, prior to advancing or retiring, ensured that they would indeed fill his or her role upon their departure. In other cases, their supervisors stepped in to certify the participant's value and her contributions to the role or for upcoming promotion. For others, supervisors built a strong relationship with their team and in doing so, gained awareness of their strengths, career aspirations, and educational goals before using that knowledge to connect them with more opportunities for growth in the future. As Sybil explained, 
You need to make sure that you have an advocate. By that I mean, that person who is aware of your strengths, and that person who will remember you when opportunities arise. I thought about that very importantly.

For one participant, this meant an opportunity from her supervisor to originate a new job after approaching him with ideas for change in the department. From there, she was able to select the title, build out the job description, and specify the oversight of initiatives to advance her skills and the overall productivity of her unit.

Another participant reported an experience early in her career when she received an external job offer; her supervisor and several organization leaders approached her immediately and said, "We don't want to lose you, so you name your price. What do you want to stay here?" By choosing to stay, she received a progressively appropriate title and entry to the senior leadership team in her division, an experience that she attributes greatly to having prepared her for a future target position.

One participant recounted an instance when her then-supervisor, who was very familiar with her career and educational aspirations, passed her name on to a professional recruiting group. At the time, she was searching for a role specific to her clinical training that was affiliated with or based near an institution where she could enroll in a doctoral program. Soon thereafter, she relocated and was working "in a leadership role based on specialized knowledge and skills" while taking doctoral classes at a nearby university. She attributes this step on her career path directly to the intentional support and proactive recommendation of her former supervisor.

"Opportunities were brought to me, and I took them," Rita shared when reflecting on her career path to an administrative leadership position. Twice during her time at Mid-Atlantic University, Rita has been asked by her supervisor to take on a new role and even after 
encouraging them to search externally, they made it clear that Rita was the intended candidate for the position:

I advised them to recruit somebody from the outside. That wasn't because I wasn't interested, necessarily, but it was a good - that was an exciting gig, and so I thought it was a way that we could use it as a carrot to recruit somebody. They said, "No, we want you to do it." I said okay. Then when (name omitted) offered me this job, I told him the same thing. This is a very fun role that I have, and so we could've lured somebody again. I'm, like, "Go get somebody really good." [Laughter]. He said, "No, we want you to do it," and so I landed in this role.

For one participant, it was her supervisor's career move and support that she believes helped her advance mid-career. They had a strong supervisor-supervisee relationship and when he announced his departure, she realized that her current job would not be the same without his influence. With his help, she interviewed for a position in his new division at a different university and weeks later, made the cross-country move to continue advancing her knowledge of the field under his leadership.

Molly recounted an experience with a former supervisor who has been supportive throughout several years of her career at Mid-Atlantic University. She appreciated this supervisor's offers to assist with matters outside of the general day-to-day responsibilities of her job and for helping her to navigate a new environment because, "a part of higher education is knowing how to navigate and who to [sic] - what experts you can connect with to be able to reach your end goal."

It was not until she came to Mid-Atlantic University that Veronica found a supervisor for whom she felt deep respect. Their working relationship helped shape her as a professional by providing space to explore a concept she had been struggling with: how to derive personal 
success from an organization where she believed being an outlier was discouraged. She highlighted his support of her autonomy:

He was part therapist and part boss, but he really allowed me to do things my way. He didn't really expect me to automatically conform my style to fit his communication style. He recognized that we were two very different people. He is also one of the few bosses I've ever had that has been willing to actually help me to deal with a bully situation in a job and encouraged me to do it.

Based on these narratives, the mentioned career advances would not have been possible without their supervisors' support and advocacy, almost all of whom referenced in this section were identified as men. Acknowledging the deficit in the number women who hold high-level leadership positions at universities (Allan, 2011; ACE 2016), it is not uncommon for the supervisors referenced in this section to be men. This subset of data provides additional context regarding the research on this broad pattern; men, who continue to hold the majority of leadership positions in higher education, maintain access to the gatekeeping processes that can prevent or promote women's advancement.

Aspects of the participants' narratives supported the existing research regarding the prevalence of the imposter phenomenon in higher education working environments and reiterated the significance of a heartening supervisor to process those feelings and develop a broader understanding with (Clance \& Imes, 1978; Parkman, 2016). For example, when Rita was considered for an internal promotion, she immediately encouraged her team to search externally, as if she did not have the qualifications to be "someone good" that she had hoped they would hire. To another participant, this support from a supervisor meant encouragement and understanding, allowing her to be her authentic self in the workplace even if that meant being an “outlier." Sometimes, the imposter phenomenon can cause high achieving individuals to 
internalize their success and question their qualifications, just as these women experienced (Clance \& Imes, 1978). Through encouraging mentoring relationships with their supervisors, they were able to build confidence and advance; this outcome supports the existing research and contributes additional narratives.

\section{Advancement Through Recruitment}

For seven of the study participants, being recruited, or as they sometimes referred to it, "being headhunted", launched their career progression on at least one occasion. Repeatedly, the participants shared accounts of receiving phone calls about inordinate opportunities that matched their skillset. Almost always, they chose to relocate in order to advance their title, career, and professional experience. Some participants had never imagined a career at Mid-Atlantic University until they received a call from a professional recruiter or for some, directly from MU's current President.

According to one participant, there were several positions throughout her career path for which she was actively recruited. For one specific role, she was recruited on two occasions; during the first, she knew that the timing was not right and determined that staying in her role was the most sensible career choice after seeking counsel from her network of mentors. Years later, the position opened again and when approached under new circumstances, she decided to relocate and accept the role; she still holds the position today and values it greatly.

Speaking of her current role at Mid-Atlantic University, another participant mentioned being pleasantly surprised by a phone call regarding a vacant position among the university's leadership. She explained that all of her prior career experiences had led up to this phone call from MU and after reviewing the position, she knew that she "checked off all the boxes" and made the decision to move her family to MU's college town to take on this new role, which she believed to be a culmination of her professional experience and skillset. This account, and her 
admission of symbolically checking off all of the boxes, reinforces existing literature related to women's patterns in human resources and job applications processes. Women are much more likely to refrain from applying for a position until they are confident that they meet all or almost all of the requirements (Mohr, 2014).

\section{Persistence}

Throughout these stories, the women humbly identified moments of persistence as they made difficult decisions, overcame unexpected challenges, and found creative ways to continue moving forward in their lives and careers. For four of the participants, a step forward meant taking a leap of faith to trust the process; they were placed in an interim position with a quiet promise that they would be become official a year into their work as the interim. In these cases, the women credited their prior interviews or professional reputation to have preceded them and they were each able to remove interim from their title about a year into the positions.

According to Susan, most of her career advancement occurred as a result of finding a new challenge to solve. It initiated early in her career when she recognized an ongoing safety issue in her workplace and realized that she did not have the appropriate professional clout to raise a concern or to help create a solution. Immediately, she decided to go back to school to earn her master's degree in order to return to that workplace in an administration-level position with access to decision-making capabilities, in hopes of making the changes that she had not been able to access before.

In exploring her career path, Veronica said, “I've outlasted, outwitted and outperformed everybody else. I was the last man standing... You have to survive the longest." For her, reaching a leadership position she had aspired to, in a men-dominated work culture, required persistence and dedication. 
In Angelina's experience, career persistence meant taking every opportunity for service early on in her career by exploring all facets of her then-working environment, pushing herself into new territory, and volunteering for committees and task forces, and as she said, preparing herself to better help people in the future.

Speaking to her representation as one of the only women in her current working unit, Marge said,

When it comes to sending people for training, the women are never considered to benow, years ago, I went - I have paid so much of my personal money to go take courses or to get my instructor certificate in something because they wouldn't because I wanted it. I seen the value of it [sic]. They wouldn't send me. Throughout my whole time at the university, I have paid to go to classes myself. I have paid for certificates. In reflecting on her career now that she is nearing retirement, Alice confirmed the importance of being flexible and persistent:

I've just been persistent. "Yet, she persisted." What it means to me is that we women really have to uplift and support each other and do it in visible ways. That means amplifying another woman's voice at times, calling out her accomplishments. It's easier at my age and station because I'm not angling for that next position. It's harder if you see her as a competition, as a competitor for the next position that you want. I would also say be prepared to move. This is not necessarily where you're going to climb the career ladder. Most people need to move to go up a level.

Persistence was conceptualized in different ways by the participants in this study: for some women, persistence was constructed as an individualized effort. For example, some participants sought additional education necessary in order to have a more powerful voice within a men-dominated unit; for others, persistence equated to longevity - outlasting the other men in 
the unit. Still, other participants viewed persistence as a collective effort in which women needed to support each other to advance to higher leadership positions and in some cases, recommended an open mind to moving, asserting that to advance, women might likely have to relocate.

For these participants, persistence was largely described as a way to increase their power, whether in a formal position or in a decision-making process. Their stories revealed the strategies they employed in order to reach a level playing field with their men counterparts or to surpass their men colleagues to advance professionally. To some participants, the ability to match men's power was unlikely to occur in their unit or at their institutions, and instead, they were forced to relocate in order to find a more equitable work environment and a position that was progressively responsible.

\section{Positive Influences}

In speaking about their career trajectories and current working experiences, the participants identified a series of positive influences, including mentorship, role modeling, building a network, and growth through leadership programs.

\section{Mentorship}

Whether formal or informal, mentorship played a significant role in the advancement of these women. Each participant listed at least one mentoring experience which they believe to have had a positive effect on the career paths to their current roles.

In Susan's experience, mentorship was exceptionally instrumental, and she reflected the importance of authenticity in mentoring relationships.

I had professors along the way that literally were my mentors. I believe huge in mentorship, which has helped me get to where I am today. That mentorship can be coaching, it can be somebody that you admire and with whom you can be real. 
For Rose, serving on a search committee led her to an impactful mentoring relationship with someone she is still in close contact with today.

She was a really good influence for me... She did a nice job of pulling me in and coaching me. "Slow down and do this, and don't be so direct. Remember you're not a consultant. You work inside this organization.” She was really good at helping build coalition. She was good at selling ideas and supporting the team. She also was a good boss and that she delegated very clearly... and let us go do what we needed to do, but I had the chance then with her to have a good mentor and a boss... I think she set me up with the right mindset to understand where I would need to be successful at MU. Molly reiterated the supportive nature of the Dean she has reported to for several years; she said he has been a great mentor to her in terms of her career aspirations and throughout the nature of her current work. Molly also mentioned the positive mentorship offered by two women, McDonald (name changed) and Grey (name changed), who have served as Provost at MidAtlantic University:

McDonald, who's no longer here, she was also fantastic. She and I had several conversations about how she was the only woman at the table a lot of times when they were at that executive level. I think, for her, knowing that, okay, there is a female voice, and there is someone who's looking out for women, and trying to bring women along. I think I would say, probably, the same thing for Grey. I think that it's nice to see that women have a voice.

Miriam detailed two mentors at another institution that shaped her growth. The first of whom devoted time to being a "great mentor" and was "kind and supportive." Miriam also referenced a dear friend and mentor at the same institution who was a volunteer for the university; through this mentoring relationship, Miriam explored a new perspective on feminism 
and utilized her feedback and advice to adapt her professional practices and inform a new approach to her work.

Also recalling her experience at a previous institution, Eleanor explained that she's had "very good mentors" throughout her programs in higher education. While working at a smaller college, she participated in a formal mentoring program while also cultivating informational mentoring relationships; these mentoring opportunities were "exceedingly helpful" and offered more than just career mentorship.

Cassandra credited one of her mentors for encouraging her to identify early-on what her career aspirations are and specifically, what type of problem she deeply wanted to solve through her professional route. When Cassandra started her dissertation, her mentor asked, "What problem are you trying to solve? It's always about the problem," and that question has served as a guiding light to her ever since. Recalling the impact of mentorship on her career, Cassandra said:

I cannot emphasize enough the mentorship. I cannot emphasize enough on that level of support particularly for me in the early 2000 s to this day... and a sisterhood. I've had the great opportunity to connect with some amazing women within our state and around our country who are likeminded and are trying to do the same things that I am. We may do it in different ways, but we all have a similar end-goal.

In Helena's experience, advice and mentorship paired with autonomy in a former role allowed her to grow as a leader. She said:

I got a little bit of advice and mentoring, but I mostly was allowed to do my own thing which, in many ways, is a good way to learn how to do something like administration because your constituency - it's like students-your constituency will tell you when you do something wrong. 
Alice recalled several mentors along her professional journey, many of whom were men. In every case, what those mentors did for me was increase my knowledge, which increased my confidence. They weren't mentors in the way of saying, "Well, this is how you should act or be or anything." They were mentors in that they trusted me to learn this and taught me and didn't give up on teaching me. They taught me in a way I could understand.

Participants also mentioned the process of offering mentorship to women. For Marietta, her own mentors led to her interest in mentoring others. She said, "More and more, as I got interested in the mentoring piece, I do enjoy mentoring people who want to be leaders." For Cassandra, the process is about creating new opportunities for her mentees. She said, "Sometimes if there's not a seat at the table for you that you want to be at, Shirley Chisholm said, 'Bring your lawn chair.' How do we bring our lawn chair?’

Evidenced by these narratives, the influence of mentorship was fundamental and, in many cases, indispensable to the sustaining success of each woman's professional career path. Naturally, the experience and impact of mentoring relationships was unique to each participant; for some, it involved the support of the two women who most recently held the position of Provost at Mid-Atlantic University. For another participant, this meant finding someone who would provide honest, direct advice while also modeling strong delegation, all of which she attributes to have prepared her to lead at MU. While some participants sought mentorship through formal mentoring programs, others developed organic mentoring relationships through classes, departments, and professional networking. In every case, the participants remember clearly what their mentors saw in them, how their mentors made them feel, and what those mentors have inspired them to do today. 
These data support and extend the existing research related to formal and informational mentorship programs for women leaders in higher education; there is in fact no question of the importance of mentoring relationships for women who aspire to lead colleges and universities (Dunbar \& Kinnersley, 2011). Considering the preparation and recruitment for women university presidents, mentorship is invaluable (Brown, 2005). Research suggests that universities benefit greatly when investing in formal and informal mentorship programs (Jackson et al., 2017); as identified through the perspectives of the participants in this study, it is clear that there is a welcome culture for mentorship, both formal and informal, at Mid-Atlantic University, and similar organizations.

\section{Role Modeling}

The participants highlighted scenarios when they have looked to role models for positive behavior cues to replicate in their personal leadership; in some cases, participants also shared examples of modeled behaviors that they took notice of to avoid replicating in practice.

Positive Modeling. When working at a nearby university, Veronica found her former supervisor to be a positive role model whose style she tried to emulate:

She is actually a person that I've tried to aspire my leadership style after because she managed to walk that line between being friendly and being a friendly person and being a boss. She understood that you didn't have to be a tyrannical asshole to be a leader. Right? It's something that I try to emulate in this, "We're all in this together. Let's figure it out." For Angelina, it was her elementary school principal whose example she has followed since an early age:

She made every single person that went to (school name) know that they were respected, and they were loved. She expected nothing but excellence from us. She corrected us when we were wrong. She celebrated us when we were right. I think that that's how we 
should all treat each other and how we should make it clear what education is. She was very astute to that.

Angelina recounted that she stayed in touch with her role model for many years, exchanging letters and support throughout her career. Though her mentor has since passed, Angelina continues to look to her as a steadfast influence on her life and career.

Guided by her lifelong mission to ensure that students feel valued and supported, Sybil found positive examples in her department at MU, specifically those in associate dean positions who became "pretty strong role models". She said, "Their ability to affect change was powerful. I thought, oh, this is something that I would like to do."

For Miriam, there were women in several of her working environments whose positive influences impacted her career:

There were so many women that I met that inspired me in different ways, that they probably didn't even know it. You know, it wasn't this formal relationship, but it was just hearing their stories, seeing how they got through something and how it would either relate to me, or how I could see me changing my vision a little bit, of how life could be or should be, was one.

Earlier in her career, Marge looked up to a woman whose story she had heard in a women's leadership session.

She was from Lewis County, which is right next to where I'm from... She was also from a very poor family and she really wanted to be a doctor. She worked really hard to get the grades and went to school and did all these things, and she accomplished becoming a doctor. It was strange about how, when she went back home for holidays and things like that, her own family gave her attitude because, “Oh, you're a doctor.”... It mirrored my 
life. I was like, "Wow, that's pretty impressive," because, if you really have that, you can do anything.

For Marge, this memory is linked with her belief in the opportunity of education, and Marge has since furthered her education as a result.

Emma reported that she frequently watches other women to identify and replicate their strategies, especially while observing facilitation tactics for faculty meetings. She said, I'm trying to really watch like a hawk what (Provost) Grey does when she's talking to the faculty... I watch Milly (VP for Creative and Scholarly Activity) like a hawk and some of these other women, what they're doing when they're addressing faculty members because I find that no matter how I say it, that I'm going to get kickback in ways I just don't expect, just pushback on some element that I would never think would've been controversial in any way.

One participant, who is involved with Staff Council at Mid-Atlantic University, identified the woman who serves as Board of Governors Representative to Staff Council as someone she has looked to as a positive role model:

She had been part of Staff Council for many years and seen different aspects of it and was able to do things for employees as you go along. She represented herself well that her voice mattered so that, when she says something or reaches out to someone, they actually take her calls. She's able to state something in a nice way but gets the point across.

Reverse Example/Negative Modeling. In several cases, participants expressed learning by reverse example, observing behaviors and taking reference to leaders' actions that they did not want to replicate. 
Marietta has "learned by reverse example" ever since she was a child. "Attending to things that didn't work well were often very salient. That would impress upon me how I'd want to do something differently." Miriam shared a similar perspective, "There are also some that I knew I didn't want to be like, right, that really helped form me into, this is not the kind of leader I ever want to be.” Miriam continued,

One of my first bosses, she was a great boss, but it was, you know, back in the early '90s, and there was certainly a time where women still felt like they had to act like a man to be taken seriously, or to move up the ladder. There was a lot of vibrato that I just thought was unnecessary. I know that on the other side, she was a really kind person, right? It was this juxtaposition of trying to be this big boss, when in reality it probably wasn't who she really was, and how she handled some things, but felt that need to be at the table to act more tough, and, you know — not careless, but not as thoughtful for employees, and things like that, you know, more brisk.

Miriam, whose approach is one of care and authenticity, also noted a supervisor whose philosophy was to take a lot of credit while not doing a lot of the work. She took note early on never to lead a team with that approach, and from another supervisor later, learned that she will always value kindness and grace when leading teams:

I've had another boss who—-she's tough. She's very difficult to work for, very difficult, to the point where you always had your phone, because if you weren't at your desk and answered the call, it'd be, like, "Where were you, and why aren't you working?"... She had a tough boss, I'm not sure if it was sort of that as she dealt with it, in turn sort of beat up on her team. I just thought, well, she's beating up on me, and I don't beat up my team. There was just something there I just thought I will never, never be like that. I've had 
both really good mentors, and mentors that I've learned from all the things I don't want to ever be.

For Angelina, learning how not to lead has also been integral in her leadership development:

I think it's important — you have to look at how you don't want to lead just as much as you want to lead. I have had experiences where other women-that ain't [sic] how I'm ever going to treat somebody that works for me, and I've said that to myself, and I try to hold true to that. Just because you have a title does not mean you disrespect other humans. Just because you have a title does not mean it gives you the authority to come off in a disrespectful manner... I think that it's very important to understand where you are, and what you're doing, and how you represent leadership for women right now in this moment, not your next, not once you get your Ph.D. but how you represent leadership right now.

As evidenced by their narratives, examples of positive and negative role-modeling were influential in the leadership and career journeys of the study participants. For example, some participants remembered role models from as early as elementary school whose ethics have guided them ever since. For other participants, there have been academic leaders who have served as practical role models because of their abilities to enact positive change throughout challenging environments and crises. Participants also referenced specific situations of reverse or negative modeling, in which they have observed behaviors or tactics that they committed to never replicate in their own leadership.

In some cases, participants referenced how they continue to rely on other women leaders at the university to help them acclimate to new environments, whether in a new role in their department or serving on a new taskforce; in these cases, the participants reiterated the 
importance of having other women in the room to look to, to learn from, and in some cases, to literally replicate behavior from when they are unsure of what to do in a challenging scenario. In regard to positive modeling, participants mentioned recent university Provosts, both of whom were women, as well as other women Vice Presidents.

Women are outnumbered in the university's leadership 25 to 16. Prior research has indicated that the underrepresentation of women in top leadership roles emphasizes the entrenched beliefs that only men have what it takes to hold those positions and hinders the leadership identity development of women within the organization (Ibarra et al., 2013); these data, combined with the narratives from the participants, confirm that the university must prioritize a commitment to a more gender-diverse leadership, with a stronger representation of women at various levels of the institution to ensure the identity development of women leaders is not being hindered by lack of representation at the top.

\section{Network}

Though methods of building a network, using a network, and networking are unique to individuals, participants repeatedly shared the importance of recognizing and using a personal and professional network to be advantageous on their career paths.

In discussing mentorship and the power of her network, Susan referenced advice from James Collins's (2001) book, Good to Great, which recommended finding a circle of 10:

Look it up, read it, and those are 10 people in your life that can call bullshit when they have to. Personally, professionally. They might be family members. In my 10, I had zero family. I didn't want family in that. I had a college roommate in it. I had a recruiter friend... I had two professors that I stayed friendly with over time. Some of them are like big brothers to me... My college roommate... One was my childhood best friend. We've 
known each other since... we were 14. That's a long time... Those are the things that help undergird, and help you know who you are.

Alice reiterated the importance of a network:

I think if that's what your career goal is, one, network, network, network. Be intentional about how you're networking, not just showing up at things, but really connecting with people, people higher up, but also within your field around the country.

For Marietta, it was the cohort of faculty members in her department who have had the most substantial influence in her network, and for Eleanor, building and using her network was one of the most significant positive influences on her career path. As Cassandra said:

I talked about those tacit rules, but it's also about who you have in your corner, who is your sponsor, and also how they're received by upper leadership to grant you access and opportunities to those higher-level administration pieces, and also getting the right training, having the right support group.

Ginny acknowledged support groups in a network she has built at Mid-Atlantic University and in communities where she previously lived and worked:

I have two little support groups here, which is really great, especially given all the craziness. I've just been lucky to just land in places with presenting and teaching... I learned a lot doing those things. I have a big network because of it.

As the literature has indicated, women's paths to higher education leadership are wrought with unique challenges, with disadvantages and barriers accumulating over time in their working environment (Ellemers, 2014); networking remains a common obstacle for women, often a challenge associated with navigating the perception and reality of the 'boys clubs' and 'old boys networks' (Broido et al., 2015; Searby et al., 2015). In navigating the environments where natural networks are established for men and 'boys clubs' remain, participants recounted 
methods of building their own networks. The women acknowledged the positive influence of a supportive network and each positioned their network uniquely on their own career path. For instance, some participants built a network by connecting with a group of people who could help provide access to future opportunities through training and connection; some participants found their networks by forming support groups when they changed jobs or relocated. To some participants, a network was defined as a group of people to hold them accountable to their authentic selves over time. The participants' narratives, combined with this literature, showcase the importance of networking, and networking for women on the journey to higher education leadership careers. For a number of participants, networking opportunities arose from participation in a leadership program.

\section{Leadership Programs}

For more than half of the participants in this study, a targeted leadership development program was self-identified as a vital aspect of their career path to an administrative leadership role. In Alice's experience, a program called Lead 21 provided exposure to various leadership styles:

One opportunity I was able to take advantage of early... to attend leadership training called Lead 21, Leadership for the 21 st Century... In that, that was a really good training experience, and I learned that there are different kinds of leadership, and there's leadership based on expertise.

For another participant, it was involvement with a nine-month intensive program called Executive Leadership in Academic Medicine (ELAM). After being nominated by her Dean and selected to participate, she joined 59 other women in Philadelphia for three separate weeklong experiences. The participant described this program to be incredibly impactful and the women she met during the training have become lifelong mentors and friends. She said, "The group at 
ELAM - that group of women have been terrific in this past couple of years, really — we mentor each other. It's a really powerful support system, so now I have them.” Additionally, this participant linked major career takeaways to her experience in the program:

Again, it went back to some ELAM training because many of - all of the basic science chairs that report to me are men, and the bulk of them older than me. They're terrific people. I had to decide to make two leadership changes, which was really tough, to be quite honest, to take established faculty members out of leadership roles and replace them — was difficult. The reason I bring that up is I think one tactic that I've realized I have to employ is making changes around me regardless of how difficult they are to make.

Women's Leadership Initiative. For five participants in this study, the Women's Leadership Initiative (WLI) played an important role in their career development either as participants or as members of the steering committee.

When citing positive influences on her career path, Emma listed the WLI as a fundamental opportunity:

Number one, absolutely the Women's Leadership Initiative at MU, far and away the most important and influential support system and inspirational factor in all that... As you know, there are different skills that have to be employed when you're a woman leader versus being a guy. There's [sic] certain things you can't do that they can do and certain things that we can do that are more effective. It's really only been through Women's Leadership Initiative that I've had a chance to get that insight and watch other women leaders not just giving speeches but really interacting with them. I think that that's been the number-one most inspiring. 
For Eleanor, being nominated for WLI and later serving on the steering committee was instrumental, especially for the training opportunities and the support she found among the group of women participating. "I have to say that was one of the best things that ever happened to me. I am incredibly grateful.” She said,

I would say that the Women's Leadership Initiative did a lot of things for me. The first thing it did was established a very strong network of women that I could call on which was incredibly helpful to at least land in the right place because I didn't know exactly always who to contact specifically. There was a network. I could say, "Here's what the issue is. Who should I talk to?" Right? [Laughter] You're always one person away from figuring out the right person which was unbelievably useful.

Supporting the WLI by serving on the steering committee was also important to Cassandra. She said, "I'm always so geeked about that because something that has given so much to me, I'm able to now serve in such a meaningful way for others because it helped me so much." To her, giving back to WLI was helpful in serving and sharing her purpose with others: This is, again, where things like WLI come in for me. I was that person. I said yes to everything...This is where my purpose-Parker Palmer calls it your true vocation to serve and share, but then you don't have anything left for you. Setting boundaries for me, as a professional woman, as a professional Black woman, as a professional Black woman from a major city who may identify with a lot more folks and because my passion, but when I started to say no, it was hard but then that's when I started the upward mobility. In Sybil's experience, the Women's Leadership Initiative reinforced the need to find an advocate:

We met in small groups. We talked about some things. One thing that they talked about that I think was very valuable was the idea that as you're moving your career forward, 
you need to look to who is your advocate. You need to make sure that you have an advocate. By that I mean, that person who is aware of your strengths, and that person who will remember you when opportunities arise.

For Marietta, it was an observation of harmful behaviors towards women in her workplace that inspired her involvement with the Women's Leadership Initiative. She then began to develop and deliver training programs for WLI. Initially, she had expressed skepticism towards WLI, “Why do we have a women's leadership initiative? Why don't we just have a leadership initiative? Why does it have to be gendered?" However, involvement with the program shifted her mindset when she considered the number of similar opportunities for men embedded in society:

Then more and more I was seeing the value people were getting and the networking piece. If you take historically, old-fashioned, older times when men would go golfing together or something, there were things that men might do where they had networking opportunities that women were excluded from. In some way these initiatives at a number of institutions are like that, balancing that.

Focused leadership development programs, especially the Women's Leadership Initiative at Mid-Atlantic University, were central to many of the participants' narratives in both academic and non-academic roles. For the women who participated in WLI, the positive impact was clear: participants attributed the program for providing inspiration and insight, helping to develop skills necessary to navigate spaces as a woman, building a network, learning to set boundaries, finding an advocate, and processing one's leadership identity as a woman. Various forms of women's leadership programs are necessary for distinct, and in some cases, overlapping populations of students, faculty, staff, and administrators (Keohane, 2014); the participants' data affirm this research and illuminate potential for growth in Mid-Atlantic University's leadership programs 
aiming to reproduce this positive impact on a larger scale by reaching more current and aspiring women leaders.

With an overall underrepresentation of women leaders in academic administration, literature suggests that masculine norms and practices continue to dismiss and exclude women (Dunn et al., 2014). Women who are qualified and on track for careers in higher education leadership lack the support that historically has been able to bring about significant change (Kellerman \& Rhode, 2014). With this in mind, it is critical to consider the participants' narratives as they relate to attrition points and positive influences. Throughout their stories, the women in this study identified the affirmative influences of an effective supervisory relationship and its influence to advance one's professional path by identifying and accessing attrition points. The participants also highlighted the power of persistence, defined by their individualized effort and its power to progress their professional aspirations. Mentorship, from as early as elementary school for some participants, emerged as one of the most sustaining and influential forces to empower and drive the study participants to the career leadership roles they hold today. In addition, mentorship and the lessons and opportunities derived from it whether formal or informal, were embedded into almost every facet of the participant interviews.

Through many descriptive examples of positive and reverse modeling, the participants indicated the importance of representation and the need for more women to be visible at every level of leadership in the Mid-Atlantic University. Participants also reiterated the positive influence of building, curating, and maintaining a reliable network - one to not only provide access to opportunity, but that will maintain accountability. Leadership development programs, specifically the Women's Leadership Initiative, emerged as an attrition point as well as a network, and for some participants, a way to give back to the program that had already given a great deal to them; this program, which currently operates at Mid-Atlantic University, 
encompasses each facet of the positive influence outlined in this section and should be sustained and expanded to continue fostering community and confidence among its participants.

\section{Theme 2 - Pervasiveness of Gender-Based Discrimination}

The women's narratives in this study confirmed the prevalence of gender-based discrimination and its intense impact on their advances to career leadership. Unfair gender rules, historical oppression of women, harassment, conscious and unconscious bias, and the overall devaluing of women are engrained in these stories and the environments where these women have worked. Participants were asked directly if they believed gender-based discrimination to be prevalent in their working environments and if they employed any tactics to navigate their environment as women; meanwhile, stories and examples of gender-based discrimination were woven throughout answers during the entire interview, along with an overwhelming sense of exhaustion from women who have been fighting gender-targeted treatment throughout their entire careers.

\section{Gender Rules}

As the participants unfailingly repeated, the rules are different for men and women. There are distinctive expectations surrounding appearance, attitude, productivity, and volunteerism, and there are fewer forgiving consequences for when women do not meet those expectations. In Susan's narrative, her career path's most glaring challenge has been facing the "preconceived notions of women" and understanding the ever-changing expectations. Referencing this concept, Emma explained: "As you know, there are different skills that have to be employed when you're a woman leader versus a being a guy. There are certain things you can't do that they can do and certain things that we can do that are more effective." 
Repeating this idea and referencing this same set of rules, Rose said, "The one thing that I would say that's the hardest for me is that the rules are different for men and women." She continued:

I think the thing is... you get that organizations are built around guy rules. You're supposed to understand those rules, but you gotta be careful how you-you can't really play by those rules. Or you have to understand there's a price to be paid when you play by those rules... Actually, I do get guy rules. What I don't like is that we're not allowed to play by those rules, but we have to live in an organization whose rules are defined by those rules... People will tell you, "Don't be so direct. Don't be-_" You're perceived as bossy and bitchy, when I'm thinking, "The guy right next to me just did the same thing. What the hell?" You know what I mean? ... I think the fact that you have to check your personality if you're an outgoing direct person is annoying.

Confirming the imbalance in expectations between men and women in the professional environment, Miriam agreed:

I do think that's something that women have to be mindful of all the time... you're always watching your words, you're always checking your emotions. If you get fired up, then you're crazy, and you're acting irrational, whereas men get fired out and shout and rage, and then it's, like, well, he's a good leader. You know?

Angelina confirmed these double standards that excuse the angry outbursts of men but prohibit them by women:

I think we find ways to meet people where they are. I think women are more inclined to be more nurturing and understanding than men. They kind of charge in and just say what they want to say with no care as to how it may land on others, but when women do it, it's as if we need to be more gracious, or we need to be more this, or we need to be more that. 
It's always some type of clarification as to how we should be in our space of leadership versus, see, that we're doing exactly what you're doing. If I walked in a room and I kicked the door down like some people can do, it would be seen in a negative space. Why is that?

When Marietta received a new leadership position, she knew that it would be met with skepticism that diminished her qualifications to those of filling a quota for women in leadership roles. Though she maintained a positive focus, the incident still resonates with her today:

I knew a number of people around the country who'd applied for the job as well. It was a great job to get. There might be people who say, "Oh, she got it 'cause she's a woman," thing. That always irritated me. I did not like-I really didn't like that, I didn't. It really wasn't - I think I wanted to deny that there were issues affecting women. I only wanted to be judged on my work, and not have anything special for a woman. I tried to push that aside. Yet, I was exposed to many misogynistic people, really bad. I think for a long time tried to excuse that, tried to see the good in those people in other ways.

Later in the interview, Marietta returned to this concept:

That's a way of just demeaning, so whoever gets selected you have diminished that woman's accomplishments, and her credentials for the job by saying, "Oh, it's 'cause she's a woman." It's the same thing when people say that about people of color. You're in your position, like it's just merely affirmative action. This person isn't skilled. I think that is truly offensive and weighs on a lot of people, and why maybe some women have to fight so hard. You just have to not be-you're not just good, it's back to that super woman stuff because of that crap.

Miriam referenced a scenario when she received different treatment than her colleague, confirming an imbalance in expectations of men versus expectations of women: 
There was one incident where there was a conversation with a higher-up leader, and there's a male colleague who, if he were to get frustrated or down, it was, like, a call to rally round and bring him back up, right? This particular boss of mine had said, you know, "I need you to do X, Y, Z." I already had a hundred things on my plate, I felt like. I just pushed back and said, 'You know, I'm not sure I can meet these expectations. If this is what you're wanting, maybe we should sit down and talk, and I'm just feeling really frustrated." He brought me in, and he's, like, "What is this? If I can't give you constructive criticism, what else—how can this work?" I'm, like, "But, you know, Johnny over here can start freaking out, and we have to rally around him. I say, time out, I've got 110 things, not 100, and I'm brought in to talk about, 'maybe we should rethink things.'" That was a really big lesson for me of "I can share, but only share so much." I can share that I'm human, but only so much, you know, that I don't, as a female, get the same —at least in my mind — get this same sort of rallying around. It was viewed more as my emotions were getting the best of me, versus the fact that I actually was overwhelmed and couldn't do everything that that person wanted, and juggle three kids, and everything else. For many participants, there is a noticeable questioning of women in a leadership position when compared to the automatic respect offered to men in the same type of role. Emma provided an example of this portent from her recent lived experience:

I noticed that the guys, even if they've come up through the ranks and people have known them as colleagues, as assistant professors and associate — and then they are put in a leadership position, I don't notice at least publicly much questioning of their leadership. Once they are a leader, they are the leader, and we will all be respectful of that. What I noticed off the bat is as soon as I was put into this administrative role specifically, my colleagues that were closest to me, that in some ways know me the best, or we've worked 
the closest, have had the most resistance to acknowledging that I am truly in this leadership position. That's been something that's been confusing and hard to understand how to fix it other than to just give it time and to prove myself more than the guys have to prove themselves. For them, they're the leader. They're good to go. For me, I think it's going to take, in some cases, several years of showing the output of what I'm doing, and then I think that essentially respect of the leadership position has to be earned... Veronica shared her perspective about the experience of trying to access "a seat at the table." She said:

It's very hard for us to break apart that emotional side of things and to become almost catatonic, for lack of a better word, about how it's impacting us from a personal perspective. I basically sat there and I told somebody. I said, "You know, look. If I were a man and I had said the exact same thing and then insisted on having a voice at the table, I would have been apologized to and shown a seat. Instead, I get interpreted as being a 'pushy broad'."

Referencing the inundating and unfair expectations, Angelina said, "I do believe that there is a point where you just wish you could come and do your job." As Emma said, "I've got to prove myself more than I notice others have to. Guys seem to be given the benefit of the doubt, and I've got to come through with the goods and prove my worth over and over and over again, and no mistakes, no mistakes." Miriam explained, "I think a female and her emotions can be used against her, which is why I was telling my husband this the other day, I'm, like, 'I have to be so careful all the time, that it gets exhausting."”

\section{Appearance}


Just as the research confirmed uneven expectations for emotion and behavior among women in the workplace, the participants also discussed different expectations for men and women regarding appearance. Angelina elaborated on these heightened expectations:

We have to determine what we're wearing, how this outfit makes us look. You know what I mean? I don't think you can negate that that's real. If you're in a world where there's nothing more than men around, how you dress matters, how you engage matters. You don't want to be too flirtatious even though you're just laughing at the joke. How is it perceived? You don't want to say the wrong thing. You don't want to flip your hair the wrong way, and/or do you flip your hair to get—-there's a lot of discussions that, as women, we have to have that I don't think that men have to be exposed to, and we judge each other, as women, based on how much she laughed at a joke, or look at her dress. I think we have allowed ourselves the removal of the value of leadership based up on a whole bunch of other criteria that have been placed on us by society, and we have a responsibility to learn how to break it.

Acknowledging the pressure she feels to conform over the course of her career, Alice said,

I think I've always felt the need to make myself as attractive as possible. Not sexy-that's different— - but to look good. I do put on makeup before I go to work and do my hair. Yeah. I don't know why. Men don't have that problem... There is research that says people are attracted to attractive people. They're biased toward them. They infer smarter, better. Maybe we're just playing to that.

As Miriam said,

I think definitely being a female is still a barrier. I know that we've made a lot of great strides, and some feel like there's no more work to be done, but there's a lot of work to be 
done yet to just come in as an equal and have ideas taken seriously, or not be judged by how you dress or how you look, or your haircut-I mean all those sorts of things that you never say to a man. You don't say, "What a great looking suit," you know, they just don't get those kind of comments. Typically, they don't. I think that being judged, especially early on when I was younger, it was often commented on, my appearance.

For some participants, the combination of appearance as a woman and as a younglooking professional added a layer of disregard and at times, disrespect from colleagues. Referencing a scenario she encountered as a young professional, Penelope said, "a young female plays differently than an older female."

There was [sic] a lot of comments that he probably shouldn't have made to me as a young woman in that vein... I ruffled his feathers a little bit, probably because I was a younger female. He just did not handle it well. He just would get so upset... I thought he was going to break his pencils whenever I would say anything, but again, who knows if that was because I was younger or a female or the combination thereof, but definitely times when you were a little bit more sensitive to who you are. Again, I was fortunate because my colleagues themselves I never thought treated me any differently... As a woman in the field, you can have great colleagues, but you're going to run into people that are going to have a different perspective of you and treat you differently because of your gender.

Referencing her experience as a young professional and often, the only woman in the room, Lily shared:

I would try to make myself look older. I cut my hair, and, oh, you name it because I was always the young one in the room, right? At the time, you would love to celebrate that, but in that moment, it's hard to do so. I would try to make myself look older. It's funny 
now because people are like, “Oh, you don’t look like (age omitted).” I'm like, “Well, that's fantastic." Thank you.

When organizations operate with patterns of assumed gender schemas, the workplace becomes an environment where women are marginalized and their contributions are devalued (Broido et al., 2015). As the participants confirmed, they have worked and continue to work in environments that are stratified by gender, and where the rules are different for men and women. These gender rules were intellectualized in distinctive ways by the participants, but each perspective shared a common thread: the rules are indeed different and there are consequences for when women do not follow those rules. For example, some participants referenced the constant pressure to be hyperaware of their tone, emotion, appearance, and facial expression; other participants noted ongoing patterns of double standards that disadvantage women and diminish their accomplishments in the workplace.

In some instances, participants discussed an ongoing competition with the masculinebased norm of leadership, especially while settling into a new leadership positions as a woman, earning respect of team members, making challenging decisions, and having hard conversations with colleagues. Participants confirmed that women are very much so being held to a higher standard, with less room to make mistakes.

Because history has aligned leadership-based features with a masculine identity, women are commonly set up to face double standards, walking the line between being assertive enough, but not too assertive at risk of being judged as abrasive (Kellerman \& Rhode, 2017). When women assert power in organizational situations, they can breach conventional stereotypes of subordination to men and it is not uncommon for them to be unfairly labeled as "bitches" (Acker, 2006). The participants' narratives, which confirm the manifestation of gender rules and unfair expectations of women in the higher education workplace, validate and extend the prior research 
on this topic. This problematic culture, which favors men's leadership and hinders opportunities for women to advance, is still ubiquitous in today's university environment.

\section{Unequal Pay and Negotiation}

Though participants were not asked about the gender pay gap or negotiation strategies and experiences, what became evident in their stories was acknowledgement of the gender pay gap and the consistent undervaluing of women that reinforces a need to negotiate salaries at different levels. Their stories and their mentions of this phenomenon were informal, as if this topic is both well-known and front of mind.

According to Sybil, there is a lot of work to be done, "Women were constantly being paid less for the same work. They just weren't being considered for anything, so things have improved, but people are still not 100\% neutral when it comes to gender." In identifying one of the greatest challenges on her career path, Sybil said, "I would say the main thing I didn't know how to do was to negotiate anything. I didn't know how to negotiate salary; I didn't know any of that."

Molly echoed, "I think that even in terms of pay structure and things, I think we still have a lot of work to do." When she has had new responsibilities added to her workload, she has asked for additional compensation before taking on the extra tasks. In offering advice to women in a similar position, she said:

Knowing to how to navigate those conversations, and know your worth, and know how to say, “Hey listen, I'm taking on X amount of work. There's some value here.” It's tough to know how to have those conversations and navigate that.

In discussing her experience at a prior institution, Ginny had to identify the injustice and fight for a fair reconsideration: 
Early in my career, I discovered that my two colleagues in the parallel colleges where I was, they were making $\$ 10,000$ a year more than I was. I was raising more money and had more experience. I went to my boss, and she was like, "Oh, well, they have a family.” I'm like, "Well, I have a family too.” Anyway, they fixed it, but I don't think they would've if I hadn't made a big deal.

Echoing a similar experience, Helena said:

The only issue I've had, really, was discovering that I was being paid less than a man who was in a similar position. When there's not a high number of people to compare against, it's always hard to know what the differences are... I got a pretty good salary increase when I told them about it, but I'm still not sure whether I really have been paid appropriately... There was, at one point, in this position where I noticed that someone else had been hired at a substantially higher salary. There was a good reason for it, or there was a reason for it; I'm not sure I'll call it good. It was, at least, partially, if not fully, corrected.

For Miriam, negotiation remains front of mind, even in a senior leadership position. Like many participants, she recognized the room for growth and the looming presence of gender bias in the system:

The other thing that I've learned to do better, but I still have a long way to go, is just negotiation. I'm really good at negotiating for the job. But when it comes to salary or benefits or perks that I know other male colleagues may have received and I didn't, that bothers me. Even when I have brought it up, and it's, like, well, you know, we'll take a look at that. There's definitely still this bias of, you know, this person can come in and ask me this, and we'll just give it without blinking. But if you ask for that, "Oh, we need to think about that for a while." I just think that's very—-there's still a gender bias out 
there... Even though we still have made great inroads, there's still—-there's just still a lot to fight, you know?

After noticing a \$20,000 pay discrepancy among several of her women and men colleagues who shared similar responsibilities and experience, Veronica asked her supervisor for an increase in salary. When he discredited her initial request for a salary increase, Veronica continued her research:

I literally pulled org charts and I got salaries and I did the conversion and I handed over a 13-page report explaining why I deserve more money. All data-driven. Not one emotional tear. Nothing about my husband not having work. Nothing about my son getting ready to go to college or anything like that. Nothing. Right? Then I waited.

Her supervisor's response included a pay increase across the board, only further perpetuating the pay discrepancy that Veronica had initially uncovered. "We got a pay raise, but we're still inequitably paid to the guy who's got less experience than we do running a shop smaller than either of ours... Who doesn't even have a Master's degree." She said, "all we did was kick the can."

From Angelina's perspective, negotiation and salary are linked directly to an individual's worth:

I think that we have to, as women, understand the market of what we're in whatever that looks like, whatever profession that is, whatever area of higher ed that it is in and do our own individual research when we're negotiating contracts and when we're negotiating our benefits to ensure that it mirrors what it actually is worth, not even mirror what your university is doing but mirrors what it is worth in the overarching higher ed. The only thing that they can say is no. The only thing they can say is that it's not in our budget and/or we don't believe whatever. Then, you have to make some decisions. 
In the process of evaluating one's worth and identifying if an institution or a job is the right fit, Angelina explained that sometimes, it is okay to pass on the opportunity:

I think that, oftentimes, we, as women, don't really understand the power that we do bring to our space, and we say yes before we recognize "not this one". This is not the university for you or the other-or whatever it is, a company, corporation. If they don't value you at the beginning of your hire, then they're probably not going to give you what you're projected to receive in the remainder of your hire. I do think it's important that when you're sitting down for first thoughts of negotiation that you value yourself appropriately. Now, oftentimes, we don't want to miss the opportunity so we'll lowball even ourselves, and so it comes down to an internal dialogue as to what you believe your worth is and what you believe you should raise to be your value even if you can't find a number that supports it throughout our higher education system.

Participants mentioned unequal pay and the important of strategic negotiation skills throughout their interviews, though they were not asked specifically about the topic. Of the participants who mentioned unequal pay or negotiation, the age range varied greatly, including the youngest bracket; this pattern would suggest that younger women are still being impacted by gender pay discrepancies. In almost every case, participants positioned salary negotiation as a challenge they have had to overcome on their path to reaching the career role they hold today, almost as if it were a rite of passage for women pursuing higher education leadership positions.

Typically, women believe that pay and promotion are necessary tools of management in that employees find it meaningful to have opportunities for growth and to feel valued within the workplace for their talents and skills (Rosener, 1990). Fostering this type of supportive culture allows teams to perform more effectively when they like they are authentically a part of the organization and can share in its accomplishment (Rosener, 1990). Considering this research, 
and the pattern for participants in this study to continuously reference unequal pay and the need to negotiate without prompting, it is clear that gender-based discrepancies in pay are very much still a relevant challenge in higher education leadership, at Mid-Atlantic University and other institutions.

\section{Hiring Processes}

Separate from salary and negotiation mentions, participants also referenced gender bias associated with recruitment and hiring processes. As Sybil pointed out, her department seems to be on the right track but there is noticeable room for growth in other units:

I actually felt like my department was very good in that respect-but I have seen in other departments where there was distinct bias against women. They were holding them to a different standard. There are some departments that have had really a real dearth of hiring of women at faculty levels. They hire them like instructor positions, but not in the tenure track.

For one participant, there is a current climate of gender-based discrimination baked into the recruitment and hiring in her school:

The school within where I work has recently, under its new leadership, the school, not the college, started hiring only men... in the past seven searches that we've had-I think it's somewhere of a total of 21 finalists... Okay, 21 finalists, only three of them are women, only 1 woman hired. We have six dudes that've been hired, one woman, and this has happened over and over and over again. I brought up specifically to the leader in charge of that that not only was this starting to happen, but he had put together a search committee of all men. Then of course they hired a guy... I feel like I spent all my political capital on that one conversation, and I think there's been little punishments all 
year long in any way that there can be, and I feel like I can't bring it up or talk about it anymore, or those little punishments will be there.

She continued by elaborating on her fear of reporting and why she only feels comfortable verbally reporting similar scenarios once a year:

Now, I found other avenues to keep reporting it for sure, but I feel like I get to make one big stink a year, and then I better be quiet for the rest of the year. Maybe that's not true, and there's other people that won't have any kind of problem with that, and they keep having their voice heard, but the little punishments that are, to me, very much connected to calling somebody out on this issue are not necessarily worth it to me to make that stink again. I'm seeing, unfortunately in my particular school, things making a turn for less diversity in terms of hiring of women. There's perhaps more diversity, little bit, in hiring people of color, but they're all men.

Participants highlighted patterns of gender bias in hiring and recruiting processes over the course of their careers and within their current working environments at Mid-Atlantic University. Some women noted healthy progress for recruitment and hiring of women in their department while pointing out that other departments still have significant room to grow. Gender typing, or the presence of gender in processes, practices, and distributions of power, can predict what types of people, specifically men versus women, might be hired by an organization (Acker, 1990); participants in this study confirmed instances of gender-typing that are currently embedded into hiring processes at faculty and administrator levels, and confirmed that there is fear associated with reporting these troublesome patterns. When applying role congruity theory to this phenomenon, it is important to consider that perceived incongruity between women and leadership roles is likely reinforced as disparity between women's gender and the organization's gender-typing increases (Funk, 2019). With evidence that gender-typing and a lack of 
prioritization for inclusive hiring practices is occurring at Mid-Atlantic University, this finding reveals a call for immediate action.

\section{Evocative Moments}

Mentions of a fear of reporting were not limited to the subject of recruiting and hiring processes; the participants continued to recall moments of harassment in their working environments and the trepidation associated with reporting. As Angelina explained,

I think that when you call it out, it's as if we're playing the victim. When you name it, people think that you are trying to create a problem that doesn't exist when, in fact, it might. I think when you name it, people treat you more like a nuisance than actually necessity to grow for them as leaders as well as for you as a leader and for whomever of the entity that you're working for. I think when you name it, it's certainly necessary at its appropriate time.

As the participants shared insights about their career paths and the many supports and challenges that they faced, it became evident that not only has gender-based discrimination been embedded in their experiences, but memories of specific instances have remained vivid and influential. Without prompting, every participant recounted a moment that has remained striking to them, a moment of harassment or targeted discrimination as a result of their gender identity. It was clear that these moments have stayed front of mind in the women's experiences, despite some of them having occurred more than 20 years ago.

When Marge participated in a professional development training for specialists in a nearby county, she recalled being asked to leave by the man leading the course.

I was the only woman in the class. When I walked in, he looked at me and said, "I think you're in the wrong class"... I stayed... After the fact, I never got my certificate... It's 
never been a problem before. I had to call and just make a big production to get my certificate because I had been in that class, and I wanted my credit. Another participant narrated an experience from early in career when she entered a new workplace:

I introduced myself to the medical director, who was a huge man, like 6'7", 300 pounds, a giant of a man with a deep, booming voice, and he looked down at me, and he said, "I don't need a dietitian. I've got a PharmD. I can't keep you out of here because Joint Commission says I have to have a dietitian, but don't cause trouble." He had had a bad experience with a previous dietitian... He was down on dietitians. I think I was probably speechless for a minute, but it was a challenge, so I had to be better than that PharmD at my job. I had to work harder. I had to learn more, to know more, to be able to justify every recommendation I made, and I learned to make the recommendations in person so there could be give and take in talking about it.

Emma also recalled a scenario from early in her career which has stuck with her for many years:

There's a very specific one that stands out as a former dean... in a conversation that I was having with him, when I said, "I really would like some more leadership responsibility," and he said point-blank to me, "In the past I put two different women in leadership positions, and they both flamed out within a year. I'm not going to do that again," just point-blank. That to me was like, "Okay, if this person is my Dean, nothing will happen for me, so just put my head down and keep doing what I'm doing," and then that situation changed. I mean, I couldn't believe that I heard that, and I've watched — that person now is in a different leadership position, and he has yet to hire a woman in any kind of leadership. He's sticking to his word. 
One participant, who had spent several years working in alumni relations, recounted her memory of several inappropriate comments and actions by alumni association volunteers:

The alumni world is sort of a good old boy network, still is... it hasn't changed much from that time to today. You know, what I see, I'm, like, damn, this has not really grown and expanded. The same conversations are happening with the same topics. I mean, I was young, probably around your age, a little older maybe, when I started there... we had a board that was elected, so members elected the board of directors... You know, we would have some that were just handsy-you know, huggers, kissers, you know. That was one where my own boss, who I loved, he was great—but he had some...challenges... He's, like, "ah, he likes ya, and he's just a good old guy, you know?" You didn't really have a recourse. I suppose I could go to HR, but again, that's where, as a female, you have to balance, do I want to make a big stink out of this, or do I get through it? The guy's on a two-year term, in two years he'll be gone, you know? I'll just avoid him as best I can when he's here.

One participant identified a moment she vividly remembers which occurred during what she thought was just a pleasant lunch with a colleague.

This person had been knocking the WLI just ever since it existed. Any time there's something about a woman or WLI, would send me emails, mocking stuff. I really found this offensive. Then one day he said something. Then referred to the group with the C word, if you know what word I'm meaning. "Those Cs". I just about died, that somebody would in this day, this wasn't that long ago, would say that... I was just beyond, that destroyed this - this person was a friend and is no longer a friend after that. There's that kind of just totally inappropriate. 
One participant referenced prior work and work-related travel with a former university president:

He reminds me of Donald Trump. He wouldn't travel with anybody else that was a female unless his wife was with him. I'm thinking he got in trouble once, and she found out. He would say all kinds of stuff and locker room talk in meetings. It was just amazing that he could even say those things and not get in trouble. I kept a log of stuff like that because I was afraid it was going to go sideways, because I already watched him go through - he went through a VP before (name omitted) got there. Then I was in her role. Then after I left, the next person lasted a year. It's like he went through about five VPs in about four years. I got a nice severance to go with it. I had a year of severance.... but I had to sign a thing saying I couldn't sue them.... I probably didn't have enough documentation to really go do anything big, nor did I really want to get into that, because I knew if I did something, I probably wouldn't ever be able to work in this field again. In detailing these stories, the participants consistently reiterated a fear for consequences if they reported harassment or unequal treatment; the women also eluded to a level of protection surrounding the men who held positions of power in each scenario of harassment. Though more women are advancing to high-level leadership positions, the highest offices still represent strongholds for masculinity; until women are equally represented in all aspects of society and until leaders prioritize and challenge inequality, women will continue to be targets of discrimination in the workplace (Jalalzai, 2008). These narratives, combined with the literature, suggest an urgent call for more women to be represented throughout the university's administration to interrupt this problematic cycle and move towards the elimination of genderbased discrimination at every level of university operation.

\section{A Heavier Burden for Women of Color}


For the participants who openly identified as women of color, the intersection of their gender identity and their race was entrenched in their stories, their careers, and their outlooks. Though only two participants voluntarily identified as women of color, there were mentions of the experience for women who identify with multiple minority groups; in each mention, the women reiterated how the challenges for women leaders in this context were and are exacerbated for women of color and for women in the LGBTQ+ community, among other underrepresented groups.

As one participant, who identified as an African American woman, shared:

I think what's often lost is that, oftentimes, women of color carry a higher burden of leadership than others because we don't just do it for our gender, but we do it for all kinds of other reasons. We do carry the weight of a lot of people with us, not just our race. It's a lot of minorities that we carry with us because we want to see doors open. We want to see opportunity provided. We want to see development of leadership. We want to see development of mindset and thought, and so I look at us as a blessing.

While the participant communicated gratitude for the role, she also explained the added personal responsibility that she believes to come with it:

I'm very honored to be in this position. I will tell you I wish I wasn't the only one in this position around leadership in higher ed or MU, but like I said to you before, it's my responsibility to ensure that I'm not the last and that we continue to look at how we increase women, in general, but most importantly, also women of color, individuals, minority, marginalized communities that have the same gifts and talents.

For a participant who identified as a woman of color, there is a cultural tax associated with her identity in the workplace: 
In our area, particularly, and when you're looking at cultural studies, they call it the cultural tax. It is the cultural tax, the professional cultural tax. It is the rent that we pay to be in these positions and to be liked.

Referencing her role as one of few women and one of few women of color to hold a position of her title, the participant reflected on the associated burden:

Having that title does open a different door. It does give me access and so having that title is important... because it also is a model, and it's what we do with it. It's a model for young women and young women of color or people of color, any historically disenfranchised group. However, it sometimes can be empty... At times for folks like me, it's a heavier burden... I think it's about your action, and what you do, and the problems that you're solving, and the way that you can build - you can get by in and really move the needle to address that problem, make some real change.

Molly, who does not identify as a woman of color, confirmed a need for change, primarily through the representation of women, women of color, and minority groups in general: The more women we can have in these positions that are breaking through some of those stereotypes and those proverbial glass ceilings... I would say the same thing about minority groups, about LGBTQ, about African American, or any person of color. I think any time you can see someone like you, I think that's easier.

When it comes to the burden of service, Eleanor specifically referenced the evidence-supported pattern in society that places a higher burden of service on women of color and underrepresented minorities. When asked about the service burden, she said, "research-based, that is true even more so for women of color and underrepresented minorities by and large, that's definitely true. Yes, I have seen it. Yes, definitely." 
Prior research indicates that within academia, the salience of gendered realities combined with enduring realities of racism is indistinguishably linked to the overall work life experience of women and women faculty (Turner et al., 2011). Participants in the study, who identified as women of color, shared their acknowledgement of the higher burden for minority women while also reinforcing their commitment to ensuring that they will not be the last to hold their positions of leadership. Their tones were honest, yet hopeful; while they see the effects of heightened expectations for women and further for women in marginalized groups, they proudly carry the weight and maintain a mindset for positive change in the future.

Throughout this section, the participants' narratives have illuminated the pervasiveness of gender-based discrimination in higher education leadership environments, confirming and extending the literature related to unfair gender-based rules for men and women, unequal pay and expectations for negotiation, gender-typing embedded into hiring and recruiting processes, evocative moments of discrimination and harassment, and an unfairly heavy burden on women of color and underrepresented minority groups.

\section{Theme 3 - Further Evidence of a Service Burden}

When asked directly about whether or not they believed there to be a heavier burden of service-related tasks expected of women in their workplaces, the participants overwhelmingly provided evidence of this concept and shared personal experiences related to the continuing phenomenon. Four participants expressed that they do not personally experience an unusually extensive service burden but proceeded to justify it by crediting their specific field or supervisor; in doing so, these women admitted to being the exception. Immediately after sharing that she felt like "an equal part of the team," Penelope contradicted herself, "I do recognize that the notetaking thing is funny because whenever you're in a meeting, they always want the woman to take notes." 
Through each interview, women continued to share, and without knowing it, corroborated the stories of other participants in the process. As Marge said, 'I see it every day. Females aren't asked to participate in anything unless there is a hidden agenda to it." Noting her status as one of the only women in her unit, Marge continued, "When it comes to paperwork, they just think women are the only people that can do paperwork. They put you on a committee so that you can do all the paperwork."

Miriam's experience upheld a similar pattern in her previous and current working environments:

I could have meetings where I'll be, like, well, you can just take some notes. Then it'll be, like, "Miriam, you want to take these?" I mean, now, you know, when I was younger, I'd be, like, "Sure." Now, I'm, like, "Why, because I'm the woman in the room?" Marge explained,

Seriously, it's like they have the good ol' boy_-if you don't want to do anything, you don't have to do anything, and it gets pushed off on someone else, which is usually a woman because they know that they'll do it. It's not an honor to have it pushed off on to you, but they make you think like you ought to just be thankful you have a job.

The women also elevated concerns regarding the service burden placed on women to fulfill a token women's seat on task forces and committees. Sybil said, "If you're a woman, if you are underrepresented minority, yes, you get asked to be on every single committee." Rita recalled an incident when a mentor looked at her curriculum vitae and immediately noticed a momentous amount of committee-related work:

"We've got to get you off of half of these committees." She said, "You're a well-funded, successful, basic scientist that's a woman. Everybody's going to want you-they need 
one." Like when they would come to take pictures, to be quite honest. They wanted a woman basic scientist...

Eleanor, whose work has exposed her to related research, provided additional evidence for the ongoing phenomenon of a gender-imbalanced service burden in higher education, saying that it is, "true even more so for women of color and underrepresented minorities by and large, that's definitely true." In Veronica's experience, being the token woman on committees is something that she actively speaks out against, in the same way that she refuses to be placed on a committee that has already made its decision without all members in the room. She said, I have a real problem with just being a rubber stamp. I think a lot of committees and service groups are put together where the solution has already been made. All they want is a rubber stamp, and I don't do that.

As Cassandra shared, the expectations of her intersecting identities compound to exacerbate the burden of unfair and imbalanced service expectations. She said, ...when you are one of the few... not only token as a woman but token as a Black woman, not only token as a Black woman but also a token as a woman... from a major city. When you get that, it's, like, “Oh, my gosh, a unicorn. I have another person for you. I have one person that you can go to." Well, that adds up. It really does. Again, it is exhausting because not only are you asked to sit on this committee, or if a student's having an issue, or they want to recruit a student, go talk to this person... you also have to keep up with everything else. This is particularly an issue from my friends on the faculty side. It's overwhelming for them because, at the end of the day, nothing mattersif you're on a tenure-track position, you better publish. Service is this much. Teaching, this much.... Let's not even bring in the second shift. 
In discussing committee-related work, Rita identified a double-bind situation. She explained,

I mentor female faculty hard on this, and I encourage their chairs to do the same, not to let them be completely absorbed by service commitments, and really respect their time and their need to be in the labs and their personal time like you do their male colleagues. Then I do something very hypocritical. [Laughter]. I look around for committees, and when I see one with no woman on it, I'm all over them, like, "Why haven't you asked any,"-it's a balancing act. I don't want women to be just eaten alive because they're willing to do the work, and I do want them represented on committees that are making important decisions. Don't just put them on the what color should the furniture be committee. I want them on high-level committees_-impactful, decision-making committees. I watch it like a hawk.

Some participants speculated that this pattern's cause might be women's socialization, attitudes, and productivity, which they believe to differ from those of men. Marietta said, I really should've listened because I did take on too much. That was, again, something that went back from childhood too. It was always just if you see something, you do something. If you see something you can fix or make better, you do it. I don't know how much of that is women's socialization.

Miriam connected this pattern to her tendency to complete tasks correctly and promptly. She said, "I don't know that it's because I'm a female or if it's because they know I'll get the job done." She referred to a recent scenario in which she was asked to lead a task force not because of her knowledge of the topic, but because of her gender and reputation for productivity. She said, "I do think that if you're a female who can get things done who has a pleasant personality, 
and you don't typically question things, you'll get handed a whole lot more than some peers will."

Susan attributed this pattern to productivity as well. She explained, ...women, especially in academia, we do tend to be able because I think we can multitask really well. So we like to step in, solve a problem, and make sure that the people around us, because we tend to be nurturers, that we're okay, getting from here to here. That's just a natural. But that message plays to who we are as females. I think men are learning to understand that better and I think they're starting to accept it better.

From Susan's perspective, this service-related burden is also related to specific traits women are socialized to possess and it's the responsibility of those women to understand their strengths and value:

I agree $100 \%$. First of all, I think women are very strong problem solvers, and we're balanced, because we're nurturers by nature. Right? We are wired, thank goodness, differently than men. I'm happy for that. What that means is, if a female is in tune-so, this whole thing of knowing who you are, spending the time to know your strengths and weaknesses, I don't care if it's a Myers-Briggs, if it's an Enneagram, whatever thing that's out there that helps you psychologically for behavioral things, take 'em. Learn 'em. It's introspection that says, "okay, this is what I like, I don't like," and understand it, because then you know, “okay, how do I maximize what I'm really good at, and can share with others, and what am I not really good at, that I might need to get better?" By doing so, Susan believes that women have control over the "the things you're really good at." She said, "You can help inspire others and use those gifts. Then again, when you're looking at the introspection and you take any standardized kind of preference thing or behavioral thing." 
Prior research indicated that women bear an unreasonably large share of the service burden in the higher education setting (Guarino \& Borden, 2017; Heilman et al., 2004; O’Meara et al., 2017; Pasque, 2014); participants offered perspectives that strongly substantiate this pattern, citing examples of the expectations continuously placed on them to complete unassigned administrative tasks, serve as token candidates on committees and taskforces, and fulfill caregiving and nurturing roles in the working environment. As the participants expressed, these hidden expectations of completing additional service-related tasks as a woman accumulate over time, becoming what the research identifies to be a burden. For women faculty in particular, this burden of service-related work and the unfair baselines by which they are evaluated can have severe negative implications in the promotion and tenure process (Flaherty, 2018). As identified by several of the participants in this study, the promotion and tenure process was a foundational step in their career journeys that would eventually lead them to the administrator roles they currently hold.

\section{Theme 4 - Strategies for Navigation and Survival}

The participants acknowledged that their identity as a woman causes them to be treated differently from their men counterparts, and often, as if they are less than. In sharing these perspectives, the women listed several strategies they utilize to navigate their working environments as women.

Numerous participants admitted to being quieter and more introverted, and as a result, were often overlooked. To combat this pattern, these participants challenged themselves to be less "soft". Alice explained, "It was something I needed to learn to address things immediately to make sure that my words were clear. When you soften things, you can be misunderstood. To make sure that I communicated in a way that made it clear that you can't misunderstand what I'm saying." Miriam, who also admitted to being "more quiet by nature," said she challenges herself 
regularly to portray a more extroverted attitude. Over time, she has balanced this challenge with her natural tendency to be a listener and employed other tactics to stay true to herself in the process:

At the table, I'm a listener... I don't feel the need to speak, unless I have something new to say. Or I'm not going to just repeat things for the sake of having my voice heard. I think sometimes that's probably hurt me, because men are really good at that —even if it's been said a hundred times, they'll still say it again, right? I'm just, like, oh my gosh, like, we've heard that 16 times. I don't need another person to say it. I've learned to become more comfortable with, I'm okay if I'm not at the table talking all the time, I'm listening. I do a lot more behind the scenes, and sort of negotiation and working on relationships and being at a table, trying to show how powerful I am, or that my ideas are the best. I think I've had to learn, and I think that's still a barrier if you're on the quiet side, they'll think you just have nothing to say. You have to figure out how to let them know yeah, actually I do, I'm just not going to be pounding the table with my fist, because that's just not who I am.

In Marietta's experience, it has been easier to practice this because she categorizes her communication style as "very blunt." She said, "Sometimes women are hesitant to be as forceful as they need to be, or as clear. They want to make the person feel good. This is not about making people feel good. Sometimes you harm the group, I mean, if you're too worried about saying something, correcting somebody, or avoiding conflict."

Penelope explained her tactics to self-regulate emotion which might appear to be too feminine,

A lot of it's personality driven and also what I do. I am cautious - cognizant, I think, is probably a better word as a woman — to not come off as the raging lunatic or emotional, 
which I think some women do get labelled. Right or wrong, that's just a reality of which we live in. Most of the time, I try to be pretty calm and reasonable. Well, I try to be direct and listen to what they have to say.

Emma, who acknowledged that she is still learning and testing new tactics to navigate academic leadership as a woman, uses "we" statements when leading faculty meetings:

I'm still learning what to do, but I find that I need to make sure that I say we. I have projects that are going on, try to say "we" instead of "I" in that circumstance. Now, I have no problem taking ownership of the things I'm doing in other situations, but in that particular circumstance in talking with faculty, about "we" and to really smile and to have some humor. A lot of these things are just good leadership elements anyway, and then just be ready for an unexpected kickback on something and to try to find a way not to get immediately angry. Those are just skills that I just am having to work on, and I don't have the answer yet.

Though Sybil did not directly mention using "we" statements, she confirmed its validity by referencing how she approaches a similar challenge by intentionally phrasing ideas as if they were not her own:

This is the most annoying male trait and it's pretty rampant, and that is, they don't like any idea unless they think they thought it up. What you have to do is, you have to phrase it in a way that they think it was their idea, and suddenly they accept it.

Marge, who has worked in a men-dominated university unit for many years, relies on self-training and education. She uses her time, both in and outside of the work hours, to educate herself on ways to better serve her department and develop strong collaborative relationships in the university environment to ensure that she is the most prepared professional in the room. The concept of relationship building within the university was a priority for Miriam as well, 
especially while navigating spaces where she was one of few women. She said, "I think that's the one thing that I've learned to deal with or manage, it's just creating those relationships for people to trust me, where I follow through on things. They know that if they come to me and there is trust... I want to help them get through whatever they need to get through."

From Penelope's perspective, women in leadership positions must exude confidence more so than their men counterparts. She said, ...you have to exude confidence... you need to be confident because one, you should be confident, but two, you need to project confidence because people will not take you seriously if you don't have some confidence in your abilities... As a woman in a leadership position, you're put into the position for a reason. Obviously, somebody thinks you have merit and that you deserve to be in there. You need to make sure you believe that too, because if you don't, it's a self-fulfilling prophecy, right? You need to be confident in your abilities and move forward.

Veronica echoed the notion of being confident and unafraid. She said, "A lot of it is just not being afraid to ask questions and putting people in difficult and uncomfortable positions and being confident enough to do that."

Confidence and courage were also central in Molly's story, mainly to provide guidance when navigating the assumptions and labels rooted in gender-based stereotypes. She said, I think it's important for women, especially, to be bold and be bold in a way that is - that you stand up for what you believe in and you speak your mind on certain things, pretty much on everything... You need to be professional. You need to make sure that any of those biases that you people may have come in with, that you try to counteract them from the get-go with the way you live your life, the way that you handle situations. I think that, sometimes, we'll have perceptions of women or-you could fill in the blank-and I 
think, if they're any marginalized group, of how they're going to behave or how they're going to handle things, or maybe a woman is not as strong. If they are strong, they're seen as aggressive. I've been labeled as aggressive a lot... I try to use that as a positive. I take a label like that and use it say, "Yes. I'm aggressively pursuing, I'm advocating for the students in aggressive way," and try to use that in some way. Yeah, I think that there are some labels that are put on women that are not necessarily fair. I do think if a man had been in this position, he probably wouldn't have been labeled aggressive. It would have been, “Oh, he’s just doing his job.” I think, for women, sometimes owning that narrative a little bit and trying to make sure that you are-you're in control of your own story.

Throughout her career, Rita has relied on a commitment to stay true to her authentic self and practices which keep her mind calm:

I think my biggest go-to is not to go far, ever, from my authentic self. I don't feel compelled to act like some of my male colleagues to fit in. I do a lot of - and this'll sound kooky — but reflection and meditation to sort of stay calm in an environment that will push some of your buttons. The basic sciences in particular are still a very maledominated arena. To be in the midst of that and then to be in this role in the midst of that, you need to keep your wits about you. You've got to be true to yourself first and calm. It's not a role that has any room for drama or-so I just try to-just that, just breathe. Sybil shared a similar perspective, referencing a commitment to your own character. She said, "I don't know if women need to think about this more carefully than men, but it's really important that you remember your own goals and don't let other people define you."

For Molly, relying on her network and connecting with other women allows her to navigate challenges. She said, 
I think being a woman, I tend to seek out other strong women and try to navigate the situation or the experience with them... I will say on our campus, I think we have a lot of women in positions... I think that helps when you have people that are like you or could have similar shared experiences. It really helps you navigate it.

Within higher education, women focused on obtaining leadership roles encounter a complex labyrinth with roadblocks and barriers that must be navigated with precision (Eagly \& Carli, 2007; Reis \& Grady, 2018). While pursuing and maintaining these positions, the study participants shed light on several strategies and tactics they employ to proceed. For some participants, this involves an ongoing challenge to be more outgoing, loud, and direct, despite their more introverted personas; other participants admitted to self-regulating emotion, exercising constant caution, and always trying to appear calm and reasonable. For some participants, the tactics involved utilizing "we" statements when they knew it should be an "I" statement in hopes of gaining more respect and finding the right moments when it was appropriate to take credit for an idea.

The participants' narratives, both from academic and non-academic positions, were filled with tactics, strategies, and tricks to align themselves more closely with the masculine-based norms of leadership in order to be accepted and respected, while also carefully tiptoeing around colleagues' egos to avoid what might be perceived as too much emotion, too much aggression, or too much confidence. Many of the participants expressed the importance of proceeding with selfassurance but doing so with caution as to not offend anyone, and when all else fails, one of the participants simply recommended to try and "just breathe."

The working environments for men and women are vastly different and prior research has revealed an array of tactics, tools, and skills that women employ to navigate these environments that were structured to benefit men. These narratives authorize and expand upon this research 
while confirming that women leaders at Mid-Atlantic University are actively navigating an environment today that requires them to uniquely activate specialized skills and tactics simply to enter the space as a woman. Out of this new data emerges a pressing question: what could the women leaders at Mid-Atlantic University be freer to accomplish if they were not forced to spend time suppressing their authentic identities and hiding their skillsets to protect the egos of the other leaders around them?

\section{Theme 5 - Progress at Mid-Atlantic University}

While discussing their career paths and current roles, several participants commented on the current culture for women in leadership roles at Mid-Atlantic University. Though some participants believed there to be progress and prioritization of women's advancement, other participants acknowledged that there is still significant room for growth. In discussing the parameters for how participants were recruited for this study, Eleanor said,

I think that you will find that with many of the people that you talked to, because when I just do the list in my head of who you might be talking to, virtually all of them have either been invited or are current participants of the Women's Leadership Initiative so that it says that there's a receptivity to having women now engage in these higher-leadership positions. That speaks to me about diversity and inclusion at the university. I think that that says a lot, right, in terms of having a place at the table.

Alice agreed that the institution is seeing progress for women and their contributions, mentioning MU's current and former Presidents.

I believe MU is making space for women, and I have not seen anything at the highest levels, from President Brown right now or (former President) before him, that didn't elevate women and acknowledge the contributions of women. I think that they're doing what they need to do. 
From her viewpoint as the only woman in an administrative leadership role within her unit, one participant shared,

Candidly, I think Mid-Atlantic University has done a really good job of celebrating women. (My supervisor) is remarkable and tremendous in that piece. I don't think he gives a crap as to whether or not it's male or female. He's looking at: Are you effective? Are you a good person? Can you support our cause? I think for our President, he has proven time and time again that it doesn't matter who you are and where you come from and any of that. He's going to give you a real shot to go make a difference. If you're part of moving this institution in a good place and a positive force in our culture, then he's going to support and promote you and give you a platform. I feel that it's genuine here. I think people respect that. It's just really positive. I'm just grateful to be a part of it. One participant said that the gender-based discrimination she faced was more evident at the faculty and department chair level and not a direct trickle from the top. She explained, It's not these guys above who are holding women back, in my experience. It's a different combination of things happening more at the level within where we are. I think that presidents of universities and deans and provosts, they want their university to be wonderful, and so they aren't threatened by somebody doing good work below them. They just see it as greatness that makes us all look really good.

From another participant's perspective, there can be strong leaders at the top and simultaneously, poor leadership at the department level, which negatively impacts women faculty in particular. She said,

At the college level... it's a little bit different, and there's a couple of things that have come up. It's the college committees that have the first pass on evaluating faculty each year and recommending promotion and tenure or not. There seems to be more of a 
traditionalist kind of view at that level to the point that one man from Statistics one time told me, "Well, if you can't work 60 hours a week, you're not going to make it here in this field," which is absurd, and I told him that. I said, "That's not a culture you should be promulgating. That's not good for anyone, not men, not women." Yeah. Just because there's the right kind of leadership at the top doesn't mean it's going to impact the faculty. Another participant confirmed this pattern:

Honestly, it's not the leadership. President Brown's completely comfortable with women leaders. Look at what he's done. Right? He's had two female provosts. He's trying to put women into different positions all over campus, and I appreciate that. Unfortunately, he does it, and then he leaves us off to our own devices. Part of it is hiring the people. The other part is supporting them and ensuring that they feel like they are having a voice at the table, and also impact. Right?

Throughout their interviews, several participants mentioned the Provost's role at MidAtlantic University, which has recently been held by women. McDonald served as Provost and Vice President for Academic Affairs from 2014 - 2019, followed by current Provost and Vice President for Academic Affairs, Grey, who was appointed in 2019. Emma explained the power of seeing women hold that role while acknowledging some of the unfair treatment based on their gender:

I'm watching what's happened at our Provost level—it's been great that we've had three women in that position since I've been here... Yes, McDonald, seeing that she went from here to President, that's wonderful, and then just watching what's happening there and watching what's happening with Grey and hearing some of the-frankly, my male colleagues' little snipes at things about her that I think — that's like nothing. That's a nothing thing, and you wouldn't do it if it was a guy in that position. 
In referencing how she models her leadership after Provost Grey's tactics, Emma elaborated:

If I can do for others what (Provost) Grey and the other women leaders are doing for me right now by simply being in that leadership position and showing vision and excellence, then that's what I want to do for others that're coming after me.

It was not just on this topic that participants repeatedly mentioned women in the Provost's position at MU; throughout the participants' perspectives on the topic of modeling, and throughout their overall narratives in this study, there were several mentions of three most recent university Provosts in the context of mentorship, modeling, and gender presentation. Prior research reveals that women are still outnumbered and underrepresented in academic leadership positions (Dominici et al., 2009); further, the percentage of women serving as the Chief Academic Officer declined from 2008 to 2013 at public research institutions (Johnson, 2016). With this in mind, it is seemingly rare for three women to have held the position of Provost most recently at Mid-Atlantic University and until there are more women represented in leadership positions at MU and in other similar institutions, it is likely that women will continue to look to Provost Grey for guidance as she continues to serve as the highest-level woman leader at MU's main campus.

On the status of progress for women's advancement at Mid-Atlantic University, several participants offered acclaim for women-centered programming like the Women's Leadership Initiative, highlighted a handful of senior-level positions that have recently been held by a woman, and in some cases, praised the leadership of current President Brown for his support of hiring and celebrating woman leaders. However, participants also discussed an ongoing dilemma: having a university president who hires and celebrates women does not mean that those beliefs and values are trickling down through the university's varying levels of leadership. More specifically, participants explained that the President's priority to hire and support women 
is not shared by all units, leaders, and gatekeepers; in fact, the lack of shared ideals for hiring and recruiting women leaders is actively blocking women from advancing at Mid-Atlantic University. In the case of this study, the data would also suggest that a university president who values women leaders does not automatically equate to the necessary support required to secure fundamental attrition points at varying outlets on a women's career journey to higher education leadership.

\section{Additional Findings: Reflection and Meaning Making}

In the final phase of each interview, the participants were asked to reflect upon what it meant to hold their leadership position at MU while identifying as a woman and to share any advice they might lend to women on career paths who are aspiring to hold similar positions. Their responses were both thoughtful and comprehensive, as well as indicative that there is still room for growth as universities create more space for women's voices at the top.

\section{Responsibility for Progress}

Throughout the interviews and while reflecting upon the meaning of their current professional roles, the participants reinforced the responsibility they have for progress, both for the university to advance and for women to have a stronger position in the university's administration.

It was easy for several participants to acknowledge the responsibility in which their role entails, while it was more difficult to see themselves as one of the only women in their position or to hold top-level leadership in their departments. Some participants said things like, 'I don't see myself as a Vice-President," and "I'm certainly a senior leader at the university, but when people say it back to me, I'm always like, 'Oh, you mean me."’

\section{Being a Role Model}


To almost every participant, the significance of their current role was linked directly to a deep responsibility to serve as a model for women, just as other women had been for them. As Miriam said, "I bear some responsibility to do the right things and to try and model the right behaviors, because I know people are watching." Emma emphasized the importance of representation. She said,

To be a woman leader, it's an opportunity to be somebody that students and junior faculty can see being successful as a model of, "This is possible," and something that we can be supportive of. I mean, I think about when Hillary Clinton was running for president, and they did polls of women and how many women said, "I just can't really vote for a woman for president," or, “It's just not really—a woman president, no," and I think it's just—we just haven't seen it, so we don't know what it looks like.

Several participants admitted that role modeling as women leaders is front of mind, especially when considering the example set for other women in their departments. Miriam explained,

It's just about me setting the right example and really moving things out of the way so that my leaders can be good leaders and move on to be whatever they want to do, hopefully stick with me for a long time. But if they choose to move on, I always felt good about that, because I'd helped get them to that next stage where they want more. That's never a bad thing.

Molly also explained her ongoing awareness of the example she sets for a young professional in her department. She said,

Not that I'm saying I'm this great thing to aspire to be, but knowing that she could be an administrator in a position at a large university, and that some of those barriers that may have been there, some of us are trying to break through. I think it's important to look at- 
to be thankful for having - how the people that have trailblazed ahead of you, but also look back and figure how you can continue to trailblaze and help other people along the way. I take it very seriously.

\section{Enacting Positive Change}

Participants also described their commitment to positive change and its connection to their current leadership roles. Susan's remarks summarized this concept, "When I look at those women and I see us around that table we equally feel a sense of responsibility and accountability to move the university forward and be inclusive."

Like many of the participants, Rita believes that holding an administrative leadership role at MU is an opportunity for change. She said,

What the role means is you have a lot of opportunity to influence positive change. To me, that is the best part of the job. That really, to me, is the job. There's a lot of day-to-day tasks and projects and initiatives and - tons of stuff. That's all cool, but the job, to me, is really leaving the place better than I found it and moving obstacles out of people's way. Being a woman in this role, I think, is quite useful, to be honest, because previously, it had been men in this position. While I want to have an overall impact, I value the opportunity as a woman in this role to influence some good change.

For Helena, it has been a powerful experience to enact and see positive change at the university over time. She said,

Well, it feels good to be able to actually drive some decision making and to fix policies that I think don't work well and fix processes and so on... I am more of a doer, a worker bee kind of person. I like to fix things, and I like to have a defined space. I have felt good about having some-I would never say power, but some ability to enact changes. 
For Rose, who has spent time both in and outside of higher education, the commitment to creating change by promoting other women has remained steadfast. She explained,

I try to make sure to promote the efforts in my own staff that are women. Make sure that they're qualified. People are my assistant directors, directors, and executive directors were not good enough at that yet. I try to make sure to invest in women. Like I said, I just try to be a good example to them.

Recognizing their opportunity to forge an easier path for future women administrators, the participants in this study provided thoughtful reflection regarding their role and responsibility for progress. For some of the participants, acting on this responsibility means being a model to other women daily while finding opportunities to mentor and set positive examples; for other women, this responsibility looks like an ongoing commitment to leave Mid-Atlantic University better than they found it. As it manifests for some participants, advocating for women in their departments and continuing to promote and recruit women leaders regularly is essential.

\section{Advice to Aspiring Women}

The women were asked to share what advice they would offer to a woman aspiring to hold their position one day. The participants all answered with more than one piece of advice as they excitedly advised on practices that were monumentally helpful to them and reflected on conventions they wished they known earlier in their careers. Their advice can be categorized by three subsets: skills and tactics, support systems, and personal strategies.

\section{Skills and Tactics}

For six of the women, a commitment to lifelong learning and preparedness was integral to success on the path to career administrative leadership at a university; the participants confirmed that a higher level of preparedness is expected of women than of men and to combat that, recommended being the most equipped professional in the room. Cassandra said, 
Learn and study all the time. Understand trends. Understand not only being an expert in your field but finding ways to connect the dots across other fields and even industries because that's where the real innovation is coming. That's what helps us stay ahead of the game...Y You have to perform, and then you have to be on the lookout. You have to look around the corner. That's what is going keep you fresh and relevant.

According to Marietta, it is apposite to focus on "Learning as much as possible about everything." From Ginny's perspective, this advice manifests as a commitment to become an expert in your field. She said, "Well, I think, first of all, you have to be good at what you're doing. You have to really work on the craft of our job as well as experiencing it enough to learn the nuances." Eleanor confirmed this concept as well as applying it also to the job search: "I would ask her first to talk to those with whom she would be working and I would offer that suggestion.... making sure that she fully understood the parameters of the job and also the environment in which she would be working, right?" Penelope applied this concept to the working environment around her. She said, "you want to learn from those around you regardless of age or thing, because they may be able to teach you something. Look for those teachable opportunities where you can reflect on how you can make yourself a little bit better."

Other participants recapped the importance of a formal education, both from women who have terminal degrees and from women who do not. In wishing she had advanced her education earlier in her career, Marge said, "I think it's very important to get the formal education. Experience counts for a lot, but you do miss some things in that formal education.” Rose reiterated this concept, saying that "If you really want to spend your career in higher ed, you gotta [sic] get your PhD, and I don't have one." 
A few participants emphasized learning and understanding finance, especially at the dean-level. Marietta said, "First, understand finance," and continued to elaborate on the importance of this principle from her perspective as a former faculty member:

You would think that you'd become a faculty member, and then you don't have to just think about money all the time. All my administrative roles, I've had to do cleanup on the finance end. Understanding how a university works, how the money works at a university is really important to be able to be successful in your job. You can also then do better at helping the faculty understand how... I've seen far too many leaders rely on their business officer, and actually not be really acquainted with the books. That has led to some serious problems at this institution and a number of other institutions... you need to leave a place better than you found it, and not mess it up.

This advice was also front of mind for Alice. She said,

I heard a woman who was, at the time I heard her, she was president of New Mexico State University, and she said that if she could study — if she could do it over again, going into leadership roles, she would study finance to learn how to budget and communications. Those are the two biggest things that an administrator needs to be skilled in in her opinion.

\section{Support Systems}

As evidenced through an earlier discussion about mentorship, the significance of a strong community of supporters was apparent in the participants' narratives. While offering their advice to aspiring leaders, the women consistently repeated the power of "finding your people".

In Emma's experience, finding mentors is her primary advice for women who aspire to academic leadership positions like hers. She said, "Find compassionate, smart women leaders at 
your institution and meet them and ask them to be a mentor to you. That's number one."

Intentionally seeking mentorship was also prioritized advice from Cassandra, who said, Make sure that you selectively seek out your core group of supporters. For me, as a woman of color coming from a homogenous community, black and brown community... coming to MU, I've had to quickly learn that you don't have to look like me to mentor me. It's even better. I've learned so much from my mentors who are not like me. Sometimes, we don't understand one another but at our core, it's just there. We're one, and so it stretches you, but selectively seek out those folks.

In offering her advice about mentors, Rita communicated regret for not having formalized her own mentorship process throughout her career. She said, Identify and formalize mentors at every stage. Don't let that be a haphazard, “Oh, I ran into someone and she was such a good source of support and advice.” Really seek mentorship and formalize it. It needs to evolve because what you need now-you will be prime example. If you needed a couple people around you now, they will not be the same couple people in five years or ten years. Find mentors and let that be an evolving part of your support system.

\section{Personal Strategies}

The participants offered personal strategies and systems that have worked well for them, from how to manage the burden of stress associated with a high-level leadership position, to how to find time to show up for their families. Miriam's advice about balance summarized many participants' remarks: "Have mercy on yourself some days, and then just be yourself. You know, don't try to fit into the role that you think somebody wants you to be, because it gets hard to keep that charade up." 
While offering their wisdom, many participants came back to the idea that being true to yourself and knowing your purpose are central to continued success as women in leadership positions. As Veronica said, 'It's going to sound cheesy because I think everybody says it, but I can sit there and tell you, "To thine own self be true." The participants advised women to find the right strategies and unique tactics to help them to sustain their goals and authentic selves throughout their careers.

In Susan's career, self-reflection has been vital to her success. She said:

Set the goal first. Put together a plan, literally a plan; know where your strengths and weaknesses are. Always write them down, figure out what you need to learn so that you can turn a weakness into a strength or at the very least, get it to an equilibrium, an equal state. You may not be an expert, but you have to understand it enough, and then go find a mentor.

Susan has also relied on journaling to collect and reflect upon her thoughts and experiences: I think journaling is huge. I think it's an important thing to do. You don't have to do it every day. I would go in and out of it over time, but I have a journal. I still keep a journal. Those aha moments, or if I hear something that I think is really cool, I want to grab on to it.

Marietta affirmed the positive impact of tactics for emotional regulation, especially during and after complicated personnel situations. She said, You can't please everybody. Your own emotion regulation is really important. I've had to terminate a lot of people... Something that makes me sick. I mean it makes me just physically ill at night, but I do it. I don't think other people see how hard it is on you, and as it should be... You just have to do the right thing. Learning how to do that, not delaying inevitable because it's uncomfortable. 
Acknowledging the demands of a high-level university leadership position, Miriam shared her perspective on finding balance:

Be comfortable that sometimes it's not going to be balanced, and as a female, you're going to struggle with that all the time. Whether it's you have kids or pets or parents, or young nieces - there's another part of your life that you're always trying to balance with this. The higher up you go, the more demands are placed on you that take you away sometimes from the family side. I struggle... I still feel like I miss a lot sometimes. I do think you can have it all, I just don't think you have it all at the same time. You just have it in the space it comes to you, and you have to just be grateful for it at that moment, and then move on to the next.

\section{Seeking and Accessing Opportunities}

Several participants emphasized the importance of actively seeking opportunities for growth, for leadership, for learning, and in several cases, for learning through volunteer leadership. Noting that learning is an ongoing process, Penelope said,

You have to look for opportunities and then ask if you can have the opportunity. A lot of times, people say yes. Then, relatedly, again, in leadership skills, look for those opportunities. Ask for the leadership opportunities, but also, you can try to work on it in your personal life, so volunteering, serving on committees. I still do this today... Learning continues constantly, so there are times, and I even was on a call yesterday where I was leading the call, but somebody else was talking and really had some great ideas and leadership and vision. I was like, "Wow, so wonderful. I want to reflect on what they're saying and learn from that, and how do I incorporate that leadership style into what I'm doing?" 
In Helena's experience, it was necessary to recognize opportunities as moments that might not arrive again. She explained,

To gain experience by saying yes to opportunities and to be aware that opportunities only show up on occasion and may not show up again the rest of your career. Be prepared to seriously consider the opportunities that come along, which could mean going either way on them. For me, I needed help from my husband and my peers and a few other people to just nudge me over the edge to say, "You can do this. It's okay to change your life a little bit and try something new."

From Alice's perspective, volunteer leadership roles and networking have opened doors to new opportunities for her to explore:

Take on leadership roles in your professional organization, in your volunteer role because then you can build those skills. You can build that network. You can use that to translate into when you go to apply for another job.

Just as each participant's career, life, and leadership journey is unique, the advice they offered to aspiring woman leaders was distinctive, yet intentional; the participants offered this advice with a positive tone while acknowledging through their narratives that the journey for women aspiring to higher education leadership positions will not be one without unexpected and ongoing challenges.

In offering advice, participants recommended being the most prepared professional in the room at all times, continuing to learn, and seizing opportunities to develop new skills and viewpoints. Participants affirmed the importance of completing a formal education as well as an informal understanding of each working environment and the rules associated with it. Their narratives suggested a toolkit that includes refined skills in finance, university politics, communications, and an interest in innovation. Well-intentioned, this advice by the participants 
reinforces the existing literature suggesting that women must be more prepared as they are held to a higher standard than their men counterparts. Further, this advice by the participants affirms a double standard: regarding the degree to which women are associated with a relative lack of agency, a double standard serves as a barrier for women as they must then perform better than their men counterparts to simply be considered competent (Eagly \& Karau, 2002).

Regarding the tactics that women must employ to navigate a men-centered working environment, the participants implored aspiring leaders to be merciful with themselves; they were direct and honest in showcasing the challenges the face in their roles on a daily basis, and to succeed in such positions, they recommended emotional regulation, finding balance, recognizing your strengths and weaknesses, exploring with reflection, and acknowledging that there will be many tough days ahead. In addition, participants shared direct advice to seek and accept opportunities of every kind along one's career path; for some participants, saying yes to these opportunities was an impetus to develop their leadership in unexpected ways.

Though existing research recognizes the underrepresentation of women in high-level administrative roles at colleges and universities (Airini et al., 2011; Johnson, 2016; Oikelome, 2017), the literature fails to address any pressing explanations for this incongruity or strategies for how it can be corrected (Johnson, 2016; The White House Project, 2009). In the absence of this research, the participants' narratives in this study offered thoughtful and direct recommendations for aspiring and current women administrators in higher education to adopt while navigating a working environment entrenched with systems that favor men's leadership.

\section{Conclusions}

In this chapter, the researcher presented findings of women's narratives focusing on their career paths and how gender-based discrimination has impacted their professional journeys. Participants' narratives revealed insightful details regarding attrition points and positive 
influences, including the weight of a supportive supervisor, the power of professional recruitment, the stimulus of personal persistence, and the impact of each on the women's professional career pathways. They identified positive influences that supplemented those attrition points and supports: engagement in formal and informal mentorship, identifying role models, developing a strong network, and participating in intentional leadership development programs such as the Women's Leadership Initiative at Mid-Atlantic University. Regarding these positive influences, the participants' narratives supported the existing literature and contributed additional findings specifically related to the effectiveness of programs like the Women's Leadership Initiative and the pressing need for a more gender-diverse leadership in university administration.

Substantiation of ongoing gender-based discrimination and its negative impact on the working environment of women leaders in higher education was apparent through the participants' narratives in this study. Supporting the existing literature, women described a culture where the rules are different for men and women and explained that unequal pay and negotiation strategies are still significant barriers for women on paths to higher education leadership. Additionally, participants revealed that gender-typing and gender bias are embedded into hiring and recruiting processes at various levels throughout Mid-Atlantic University and other organizations. These narratives furthered the application of role congruity theory, reinforcing a form of prejudice that defines the perceived congruity between a woman's gender role and her position as a leader. As Eagly and Karau (2002, p. 573) defined it:

Perceived incongruity between female gender role and leadership roles leads to two forms of prejudice: (a) perceiving women less favorably than men as potential occupants of leadership roles and (b) evaluating behavior that fulfills the prescriptions of a leader role less favorably when it is enacted by a woman.” (Eagly \& Karau, 2002, p. 573) 
It is clear that this theory can be applied to the culture at Mid-Atlantic University and based on the narratives of women in this study, other universities and working environments where women are still evaluated against a men-based leadership norm.

Authenticating the prior research regarding the prejudicial burden of service that falls onto women, and more heavily onto women of color, participants in this study discussed examples of this pattern from both academic and non-academic professional perspectives; these narratives contribute to the body of research that acknowledges this service burden and its unfair implications on the workload of women. Reiterating the unique challenges that women encounter within the higher education environment, participants' narratives outlined a variety of tactics that women employ to navigate those spaces including emotional self-regulation, overpreparing, trying to appear extroverted, disguising their own ideas as the work of their peers, utilizing 'we' statements instead of 'I' statements, and finding ways to exude confidence while trying not to appear aggressive.

In discussing the environment at Mid-Atlantic University specifically, the participants expressed gratitude for their roles and the representation of other women in senior leadership positions while also stating earnestly that there must soon be more women represented. The data revealed that while a university president might support and celebrate women, it is not guaranteed that their values and support for women are being replicated at every level of leadership; as revealed by this study, prioritization for equitable support and hiring of women must be embraced at every level of a university to positively impact a woman's career path towards senior-level leadership in higher education.

While conceptualizing their roles as senior leaders at Mid-Atlantic University, the women embraced a collective responsibility for progress by committing to model constructive behaviors, enacting positive change, and offering advice to women who will come after them. Though each 
participant and her career journey was naturally unique, the women made meaning through telling their stories of their support systems, the challenges they overcame, and the barriers they continue to face as women in the workplace today; their narratives quite simply affirm the body of existing literature related to the various facets of women's leadership in higher education and contributed new data regarding barriers and political forces that remain. In the following chapter, the researcher will summarize these findings and offer a discussion of implications and direction for future research. 
Chapter Five: Discussion 
Land-grant institutions are uniquely presented with a challenge to diversify the gender represented in leadership roles, especially those endeavoring to maintain research status, honor land-grant designation, sustain high profile athletic programs, and lead comprehensive academic medicine units (Hart, 2014). In these environments, women encounter substantial barriers in various phases of their career paths that could otherwise support a more straightforward career progression to serve in senior-level leadership positions. After years of women fighting inequitable structures to simply be able to do their jobs, it is time for the cycle to be corrected.

This qualitative study aimed to explore the career pathways of women in leadership at Mid-Atlantic University, identifying the positive influences and attrition points, examining the barriers and challenges, and determining areas for one land-grant institution to grow in this circumstance. Grounded in feminist research and the principles of role congruity theory, this study placed women's narratives at the forefront of the data collection and analysis, elevating their voices to expose a clear direction for positive change. This chapter includes a discussion of the major findings related to the existing literature on the experiences of women administrators in the workplace of higher education leadership and concludes with a discussion of the study's limitations, current context, and recommendations for future research.

This chapter features discussion and future research recommendations related to the following research questions:

1. What are the attitudes, behaviors, and/or tactics that women in senior-level administrative roles employ to successfully attain (and maintain) a high-level position?

2. What are the policy, structural, cultural, and behavioral influences that impact the experience of women on career paths to leadership roles at a land-grant institution?

3. What are the attrition points and positive influences in the career pipeline identified by women? 


\section{Summary of Findings}

The use of feminist-based narrative inquiry, focusing on the experiences of individuals whose everyday activities are linked to and impacted by institutional relations, reiterates the importance and consideration of research that explores these environments where women lead and work (DeVault \& McCoy, 2002). The findings of this study include implications for its home institution of Mid-Atlantic University that can be applied at other land-grant institutions and similar universities, as well as other organizations facing the timely task of cultivating improved environments for women to lead. Categorized by each research question, the following summary outlines the findings of this study.

\section{Research Question 1: Attitudes, Behaviors, and Tactics}

The participants' narratives revealed that there is not one singular strategy to navigate a challenging career scenario or one specific tactic to employ each day when showing up in an environment where the rules are stacked against women; there is no playbook. To navigate the labyrinth and maintain forward progress on a career path focused toward higher education leadership, women employ a range of strategies unique to their character, motivation, and area of focus. These tactics enable their success in environments dominated by masculine-centered norms.

Women engage in ongoing efforts to be more outgoing, less soft-spoken, and more direct than their typical demeanor to gain respect and elevate their voices. They actively find ways to self-regulate emotions to appear less feminine. Women normalize paying out of pocket for training and education to be the most prepared person in the room; they activate confidence to insert their leadership and expertise into the conversation; and, they rely heavily on support systems to continue growth and preparation. In some cases, they disguise their own ideas as the work of their peers in an effort to seem more relatable and less intimidating; for some women, 
they go so far as to use 'we' statements instead of 'I' statements when taking credit for their own projects in staff meetings. They are constantly watching, listening, adapting, and proceeding with caution, knowing that their leadership is being evaluated on a scale designed specifically by and for men. They are employing a range of skills and survival tactics every day to seem more of one thing and less of another, balancing their peers' expectations of an appropriate level of assertiveness, and as they reported, hiding their true selves, all just to be able to come to work and do their jobs each day.

Yet, women leaders are still finding time to serve others, as the participants reported in this study. For many women, accepting the responsibility to create change for the women who will come after them is also a critical aspect of their role. Serving as a positive role model for others; enacting positive change whenever they can; and, transforming their personal advice into action to help aspiring women administrators develop their own strategies are central tenets of their belief in future progress.

While existing research acknowledges the underrepresentation of women in high-level leadership roles at colleges and universities (Airini et al., 2011; Johnson, 2016; Oikelome, 2017), it fails to produce any justifications for this incongruity or strategies for how it can be rectified in the future (Johnson, 2016; The White House Project, 2009). In the absence of this research, the participants' narratives provide direct recommendations for aspiring and current women administrators in higher education to adopt while navigating a men-dominated work environment. Utilizing role congruity theory and feminist theory to approach these data-driven recommendations allows for the problematic patterns to be addressed while ensuring that women remain at the forefront of the strategies identified to correct the culture. According to role congruity theory, attitudes can be less positive toward women leaders and potential women leaders than those attitudes towards men, and a more difficult path exists for women to become 
leaders and achieve success in leadership positions (Eagly \& Karau, 2002). If women in higher education leadership roles are actively employing tactics and strategies to simply enter a workspace as a woman, there is confirmation that this arduous path to leadership roles not only exists but prevails once women reach those roles. Further, this research identifies supports to be strengthened and augmented along those career paths as well as barriers to be eased and eliminated.

\section{Research Question 2: Policy, Structural, Cultural, and Behavioral Influences as Barriers}

The ubiquity of gender-based discrimination and its impact on women's experiences in academic and non-academic leadership positions in higher education is apparent in the current working environments and throughout the career paths women must traverse to reach these roles. Through identifying invisible gender dynamics embedded into bureaucratic structures, a feminist approach can reveal how inequality is produced and reproduced in everyday life (Naples, 2003); this study authenticates existing research related to the pervasiveness of gender-based discrimination at various levels of the higher education environment and contributed additional and recent narratives. Unjust gender rules, prejudiced hiring processes, historical oppression of women, harassment, silent expectations, conscious and unconscious bias, unequal pay, and the overall devaluing of women have been embedded into the higher education working environment and continue to cause a detrimental ripple effect on productivity, progress, and advancement for women. The weight of these challenges is only exacerbated for women with additional marginalized or underrepresented identities, including but not limited to women of color.

Working environments for women leaders at Mid-Atlantic University and other universities remain stratified by gender, with unspoken rules that are different for men and women; within these in these environments, there are consequences for when women do not follow the rules and there is less flexibility for women to make mistakes overall. Still, 
negotiation and gender-based pay discrepancies are ongoing challenges, with women regularly forced to evaluate their worth, complete research, and build a case to be considered for a pay increase; in many instances, women are discovering years later that they have been paid inequitably for undetermined amounts of time in prior positions or when it is too late.

In addition to the inescapability of gender-based discrimination that women seemingly face at every level of their careers, a related service burden continues to fall more heavily on women than men in the higher education environment. This burden, which exceeds the expectations of service in their job descriptions, manifests through silent expectations of voluntary administrative work assumed to be the duty of the woman in the room. Due to the uneven ratio of men to women in higher education leadership environments, women are expected to serve on more committees, fill the token 'diversity' spot on taskforces, fulfill more responsibilities related to nurturing such as celebrating birthdays, hosting office events and parties, and being an informal counselor to staff and students beyond the traditional call of duty.

Many women feel as though they cannot decline these service-related activities that are involuntarily expected of them and therefore, must find ways to manage their given responsibilities on top of the added service burden. In addition, while some women might be appreciative of the opportunity to access a seat at the table on certain committees and in certain conversations, they are aware that these opportunities are often only available because their gender identity fulfills a quota for representation.

\section{Research Question 3: Attrition Points and Positive Influences}

Participants credited their career advances to the impact of a deliberate and helpful supervisor, professional recruitment, and personal persistence. There is a direct emphasis on the value of building and sustaining a positive relationship with a supervisor or manager in order for women to develop skills, advance knowledge, seek new challenges, prove value within the 
workplace, prepare for the next step, strengthen reputation and network in the workplace, enter consideration for internal promotion, or receive recommendation for external opportunities. Positive relationships with direct managers can lead to advancement internally and externally, and in some cases, through professional recruitment as well. On the career path exclusively, women identified personal persistence as a motivator and launchpad for continued progression and growth.

Reinforcing the importance of gender representation in visible leadership positions, women are looking to other women for positive role-modeling; these data support the existing research and contribute new results based on the unique leadership of Mid-Atlantic University. Women will continue to look to the highest-status woman leader in the room and in doing so, will take notes and replicate her strategies to navigate challenging situations such as personnel issues and the facilitation of difficult faculty meetings. Building on the prior research, these data confirm that institutions like Mid-Atlantic University should prioritize a commitment to a more gender-diverse leadership, with robust representation of women at various levels of the institution to ensure the identity development of women leaders is not being hindered by lack of representative and relatable role models.

Consistent with the existing literature (Brown, 2005; Dunbar \& Kinnersley, 2011; Fochtman, 2011; Hill \& Wheat, 2017), each participant credited mentorship as a key positive influence in their professional advancement and success in their current roles. Mentorship, supplemented by the intentional cultivation of a strong network, continues to be a vital tool for women seeking and maintaining higher education leadership positions. Actively learning by positive role modeling and by reverse example also proved to be strong developmental opportunities that women continue to use today. In addition, participation in targeted women's leadership development programs proved to be an ongoing positive influence on women's career 
paths. Specifically, the Women's Leadership Initiative at Mid-Atlantic University was cited by many participants, and identified as an impetus for positive community, mentorship, skillsharing, learning and development, and a supportive network for the women and steering committee.

\section{Implications for Practice}

From this research, we can discern that the experiences of women in high-level leadership positions at Mid-Atlantic University are affected by ongoing challenges related to their gender identities; despite notable progress, the participants confirmed that the institution, and other universities and businesses alike, still have a long road ahead when it comes to creating gender-equitable workplaces. Existing research indicated that women are outnumbered and underrepresented in academic leadership positions (Airini et al., 2011; Dominici et al., 2009; Johnson, 2016; Oikelome, 2017); cogitating the rate at which women are earning degrees and pursuing positions of higher education leadership, the underrepresentation of women in senior administrative positions is troublesome, especially at research institutions (Dunn et al., 2014). With many higher education leaders, and specifically women, at Mid-Atlantic University expected to retire in the near future, the following questions remain: for those who are soon retiring, who will be considered to fill their roles? For those who are ready to continue advancing, how are opportunities and pathways cultivated for them to do so? And, for those women who are just now beginning a career path towards university administration, what supports can be intentionally built into the career ladder to ensure an easier climb?

\section{Building Pathways}

In this study, the women led career paths unique to their individual aspirations, training, opportunities, and challenges. As this study verified, there is no clear pathway for women to follow when seeking high-level leadership positions at a university. The findings suggest that 
while some women believed there to be a traditional path to administrative leadership in academia, they were unable to follow it and instead encountered challenges and barriers while proceeding indirectly. This perception and their narratives confirm that women focused on obtaining leadership roles are no longer facing a glass ceiling, but rather, a complex labyrinth with roadblocks and barriers that must be navigated with precision (Eagly \& Carli, 2007; Reis \& Grady, 2018). Acknowledging that women must commandeer a labyrinth of twists and turns, detours, and dead ends while men continue to follow a straighter path with fewer obstacles (Bruckmüller et al., 2013; Eagly \& Carli, 2007), there is not only room for further research but also for research-informed action to alleviate the barriers and challenges slowing women's advancement.

Initially, this study set out to highlight supports and barriers on academic and nonacademic pathways to higher education leadership positions; as the participants' narratives revealed, there is no clear pathway and further, there is no set trajectory that is singularly either academic or non-academic. Actually, there were several participants whose career paths could have been categorized as academic and non-academic at varying points in their given trajectory. After discovering this finding, the researcher shifted from trying to categorize career paths as either academic or non-academic and instead focused on telling their unique stories and sharing overarching findings related to the many facets of the participants' experiences, whether academic, non-academic, or a combination of both.

If there is in fact no clear pathway for women seeking career advancement in this capacity, the following question arises: how can we support women as they pioneer their own unique routes to leadership positions, whether academic or non-academic, in higher education? As revealed by the findings in this study, women build their own career trajectories through persistence; yet, there are barriers and challenges faced uniquely and intensely by women who 
threaten to hinder such advancement. In order to truly offer equal opportunity for men and women to lead in this capacity, those barriers must be acknowledged, dismantled, and prevented moving forward. Sustaining positive influences must be created, cultivated, and maintained over time to supplement the historic and organic influences which have been advancing men in higher education leadership at a more efficient pace than women.

Often, when considering women's leadership and building programs to support it, opportunities for men are considered as the baseline; in doing so, the results are frequently initiatives like mentorship programs, networking events, negotiation workshops, and socials, all aimed at catching women up to the opportunities that men often have access to more organically. As the literature and this research have confirmed, women are considered in a leadership capacity with men and masculinity as the baseline. However, what if there were new programs and pipelines built just for women, designed with no baseline of masculinity in mind? As the participants repeated in this study, there is no clear pathway to higher education leadership, and yet, only women seem to be having trouble eventually accessing those roles, despite being highly qualified, trained, and ready to lead, as the literature reported. This information lends itself to an opportunity for a pilot fellowship program for aspiring women leaders, as early as enrollment in graduate school programs, for women who seek interest in developing a range of skills and tactics necessary from an early career point to more easily climb the professional ladder. This study has identified intentional and insightful advice, strategies and tactics to navigate environments as a woman, professional skills, positive influences, reflections on overcome challenges, and timely data regarding the campus culture and leadership environments at MidAtlantic University. The results of this study, supplemented with additional perspectives and research, could be the foundation for a curriculum of a multi-year fellowship program for aspiring woman leaders in higher education. 


\section{Eliminating Bias at Every Level}

This study confirms the existence of gender-based discrimination and bias at every level of a woman's career path in higher education and in the current working environment at MidAtlantic University. The effects of gender-based discrimination remain front of mind for the participants and continue to inform their leadership approaches daily. Their narratives support the research that indicates how identity-based stereotypes can powerfully impact leaders' behavior by triggering anxiety and diminishing aspiration (Kark \& Eagly, 2010). One of greatest challenges for underrepresented women in leadership roles is the persistent and often unconscious bias, leading to the silent deduction that women lack the competence of a man and must work harder to reach the same results (Ellemers, 2014; Kellerman \& Rhode, 2017). This study provides additional evidence to bolster Trahan and Growe's (2012) research which showcased the discriminatory practices that women face, leading to hampered development and achievement in the educational work environment.

The findings of this study, intertwined with themes of gender-based discrimination at every level, suggest necessary and immediate change to the higher education administration working environment, not only to fix current systems of oppression but to build structures that will outlive the participants' voices who have bravely acknowledged the need for action. Unspoken gender rules, unequal pay, prejudiced recruitment processes, sexism, harassment, unconscious bias, and the overall diminishing of women are deeply rooted in the experiences of the women who currently serve as high-level administrators at Mid-Atlantic University; though many of their stories have been compiled over careers leading up to their current role, they have shed light on current problematic structures at Mid-Atlantic University that preclude not only their own productivity, but overall advancement for the university. 
As the participants identified, the rules are different for men and women, and there are consequences awaiting women at every turn when they fail to meet the preconceived expectations regarding their appearance, attitudes, tone of voice, productivity, hours of volunteerism, and obedience in the workplace. To break the cycle of this complex phenomenon and adhere to expectations associated with the double bind for gender stereotypes, professional women employ a wide range of navigational tools including the choice to accept organizational pressures while adapting behaviors and changing expressed opinions to pass as someone they are not (Ballakrishnen et al., 2019). As the participants in this study confirmed through their strategies, women try to fit into men-dominated organizations by emphasizing their own depiction of masculine characteristics and leadership styles to reinforce their advantage (Derks et al., 2016).

Based on the participants' narratives, institutions including Mid-Atlantic University must take a comprehensive look at hiring and recruiting processes at various levels throughout an institution's hierarchy, exploring gender representation on search committees, recruitment of a gender-diverse candidate pool, evaluating gender-typing and bias within the overall recruitment cycle, observing processes by which finalists are identified, pay structures that do not require negotiation tactics by women candidates, attitudes and culture in hiring departments that might impact the process, and safe methods for individuals to report discrepancies in those processes. Further, institutions must construct opportunities to address the structural barriers and issues that negatively influence the job expectations and experience of women at every level (Eddy \& Ward, 2015).

Because there is evidence to believe that certain circles and processes of Mid-Atlantic University are operating in an unreceptive and problematic manner, it is reasonable to suggest an immediate audit, involving anonymous 360-degree feedback opportunities to identify room for 
prioritized improvement as it relates to creating an inclusive and comfortable working environment that yields an inclusive and comfortable learning environment for college students. This type of audit would inform both short and long-term strategies, as well as key players, to embed the elimination of gender-bias and gender-based discrimination from the masculinecentered norms of university culture as the participants have seen it today.

Because all but one of the participants in study have worked externally from MidAtlantic University, their narratives include perspectives from other businesses, nonprofit organizations, healthcare facilities, law firms, and in many cases, from other colleges and universities. It is important to consider that many of the challenges they have faced, the positive influences they have experienced, and the factors that have led them to the roles they hold today occurred in various environments and organizations; the results of this study are not unique to Mid-Atlantic University. With this in mind, it is not unreasonable to suggest similar audits and feedback opportunities for organizations to identify areas of growth to reach more genderequitable leadership. Based on this study's finding regarding ongoing gender-based discrimination embedded into workplace systems, it would be sensible for organizations to routinely explore the biases within their processes and in doing so, build new supports that will sustain.

\section{Fostering Opportunities for Mentorship and Support}

Mentorship has served as an advancement tool for women across disciplines and industries according to the literature and the results of this study. Without question, mentoring relationships have long been identified as an accelerative tool for women on career pathways to higher education leadership positions (Brown, 2005; Dunbar \& Kinnersley, 2011; Fochtman, 2011). Prior research indicates that the majority of women administrators found their most significant mentoring relationship through an informal interaction (Dunbar \& Kinnersley, 2011). 
The women in this study repeatedly emphasized the positive impact of formal and informal mentorship experiences and recommended "finding compassionate, smart women leaders" (Emma, personal interview, July 7, 2020), seeking coaching, and eventually, offering mentorship to others in return. As the participants progressed through leadership positions, they were continuously greeted with stereotypical expectations and gender-based bias and discrimination, reinforcing a need for strong support systems and mentors to help them remain focused and positive. While the findings highlighted the resiliency of the women as they overcame these barriers, the results also showcased the advantageous influence of mentorship and a support network as they sought clarity, comfort, and empowerment from others who could relate.

The results of this study would agree with the literature regarding the need for universities to create cultures that support the development of mentoring relationships, especially for aspiring or new administrators (Dunbar \& Kinnersley, 2011). For an institution like MidAtlantic University, this could include weekly time designated for the cultivation of mentoring relationships, incentive to reward mentors for offering genuine time to others, and a central expectation of mentorship integrated in the workplace (Dunbar \& Kinnersley, 2011).

When combined with the findings related to substantial support systems, networks, and the positive influence of a capable supervisor, it is easy to identify that the burden of progress must be shared by men and women alike. Many of the participants who cited their supervisor as a positive resource for advancement were referring to a man; without that supervisor's intentional leadership, they believed strongly that they would not be where they were today. While prior research and findings shed light on the organic support systems that exist to advance men throughout their careers, it is easy to see that there is extensive room for improvement in implementing a similar benefit system for women. Often, women are told to actively seek mentors, to build their support systems, to advocate for themselves, all of which has been 
evidenced in this study, and yet, there are not nearly enough calls to action which strongly suggest methods for men to share the burden and actively create opportunities for women to be mentored, supported, and empowered among a sustaining network.

\section{The Value of the Women's Leadership Initiative}

Of the 18 participants in this study, at least five women credited the Women's Leadership Initiative at Mid-Atlantic University as a positive influence and valuable opportunity for their personal and professional growth. For these women in particular, the WLI has served as an "influential support system," an "inspirational factor," and "one of the best things that ever happened to me." As one participant explained, the WLI provided an intentional space for women to learn and grow while supporting each other, an opportunity that does not happen organically in the workplace as often as it has historically been available to men.

The Women's Leadership Initiative has expanded to include forms of training, keynote speakers, and meaningful meetings with accomplished senior leaders at Mid-Atlantic University since its founding in 2011; currently, the program provides executive leadership training for internal members of the MU community with results including improved networking, more valuable engagement, and an enriched sense of community. Due to the relevance and positive impact reiterated by the women who participated in this study, there is a growing need for stronger support, awareness, and resources for the Women's Leadership Initiative at MidAtlantic University. If the participants' lives and careers have been positively affected by their involvement in the WLI, there could be a substantial opportunity for the WLI to expand. By initiating additional cohorts, creating more opportunities for past participants to give back through facilitation and mentorship, building a segmented curriculum to target women leaders earlier in their careers, and including more voices from across the campuses of Mid-Atlantic 
University, the output could significantly expand and simultaneously, so would the overall enhancement of a more welcoming culture for women across the university's entire community.

Again, when programs for women are built, existing opportunities for men are considered as the baseline; following this pattern yields results such as networking events, social gatherings, mentorship programs, and negotiation tactics. Though well-intentioned, these programs are all focused on catching women up to the opportunities that men have already had access to. Acknowledging that women are viewed in a leadership capacity with masculinity as the benchmark, there must be critical consideration of designed programs for women that go above and beyond simply catching them up to men; future programming must even the playing field and reach women early in their careers.

These implications are not unique to Mid-Atlantic University; with almost all of the participants' narratives from this study featuring years of work and educational experience outside of MU, it is important to consider the transferability of these results to other institutional and organizational contexts. The Women's Leadership Initiative at MU provides a benchmark with proven results for positive impact that organizations can look to while considering similar focused programming, and on a smaller scale, the consideration of embedding moments for mentorship, networking, and supportive conversations could remain a priority for those organizations that are easing into women-centered programming or operating without an allocated budget.

It also must be acknowledged that in order to truly interrupt ongoing systems of genderbased discrimination, programs like the Women's Leadership Initiative must be supplemented with parallel efforts to ensure that the problematic patterns are not only halted but prevented from continuing in the future. While these programs are necessary to correct a lopsided system and these programs, like the WLI, must continue, there must also be ongoing strategy and action 
to correct that lopsided system. Though it is likely that strategy and action toward correcting the system will be initiated and led by women, it is essential to consider that women in the higher education environment are already spending extra time catching up to their men counterparts, adjusting their demeanor in the office to compete with the masculine-centered leadership norms, putting in longer hours and taking on more service-related tasks, and negotiating their salaries to be valued for their true worth; men and women alike must share the burden of correcting this system.

For example, what if instead of only teaching women strategies for navigating a problematic environment, organizations were better preparing leaders to foster safer and more inclusive environments for women to lead in? What if instead of asking women to spend additional time and resources learning how to combat unequal pay through negotiation tactics, resources were allocated to ensure that university gatekeepers and human resources teams were fully trained, equipped, and held accountable for facilitating fair and equitable hiring and recruiting processes that evaluated candidates based on their professional experience and not their identity? Based on the prior literature and the data presented in this study, programs like the Women's Leadership Initiative and strategy-based training on subjects like salary negotiation are still critical right now in order to lead women through current working environments that are very much so still harmful to women. In an ideal scenario, the culture will shift significantly to the point where leaders of all identities will share the burden of responsibility, learning new skills and tactics to create a more inclusive environment for people of all identities to lead in.

\section{Elevating Women of Color}

Habitually, women of color are numerical tokens in the organizational elements of higher education institutions and their administrative environments (Turner et al., 2011). Black women especially are forced to constantly prove their capabilities while disproving programmed beliefs 
that assume an inability to excel (Davis \& Brown, 2017). Feeling marginalized in the workplace can lead to lower levels of control over one's individuality and belonging, which are critical dynamics for individuals to feel welcomed, supported, and valued (McCluney \& Rabelo, 2019).

The experiences for women in higher education leadership are wrought with unique challenges presented as a result of their gender at every step on their career journeys; without question, these challenges are amplified for women of color, LGBTQ women, women of other underrepresented populations, and women who identify with any combination of intersecting and underserved identities. By recruiting women for this study through the aforementioned selection criteria, there were integral voices left out of this study due to the lack of representation; once the men were removed from the selection pool, a disproportionate number of women remained and among that pool, there were mostly white women.

Though only two women of color participated in this study, their voices, combined with observations of their oppression from white participants, loudly authenticated the existing research related to the experiences of nonwhite women in higher education administration positions. The participants shared stories of being the token minority woman in the room or on the committee, the burden of having to speak often on behalf of her entire perceived racial group, the loneliness and frustration associated with often being the only woman and the only Black individual in high-level university conversations, and the pressure to succeed in order to open doors for future women of color in similar roles.

And yet, their stories illuminated positivity and direction for change, citing motivation to ensure that though they might be one of the only Black women in the university's leadership right now, they will not be the last. Consistent with the existing research, the participants also directed attention to areas of growth at Mid-Atlantic University, including an acknowledgment of the cultural tax and uneven service burden placed on women of color, the cultivation and 
mentorship of young women and young people of color earlier in their career pipelines, and most significantly, the growing need for a more diverse representation of gender, race, and underrepresented identities among the university’s leadership. Over time, a more diverse workforce will naturally build a more inclusive culture; when a culture feels fair and inclusive, women in underrepresented groups are more content and therefore, more likely to thrive (McKinsey \& Company, 2019).

These challenges are not unique to Mid-Atlantic University; however, as a researchfocused, land-grant institution whose mission is tied deeply to innovation and its home state, MU is uniquely positioned to create change. The potential for impact is easy to conceptualize, especially when considering the existing literature combined with the participants' narratives regarding representation: the more women and women of color people see in positions of leadership in a powerful, visible place like Mid-Atlantic University, the more quickly it will become acknowledged as the norm. Over time, women of color, or women of other underrepresented groups, will see representation and believe that they, too, can hold that kind of position; young women will have more role models whom they identify with. And naturally, there will be more ideas, more perspectives, and more diversity around the table to create richer and deeper conversation when making decisions.

Again, this is not unique to Mid-Atlantic University. As evidenced by the participants' accounts in this study, many organizations could benefit from structural change and more inclusive leadership practices at every level. Based on the results of this study, creating places in the conversation for women of color is pertinent to the success of the organization and the future of the women who will continue to break down the barriers on the path to lead it.

\section{Reflections on the MU Environment}


The findings revealed that while many women believe there to be strong leadership by Mid-Atlantic University's President, there is not always a direct downstream effect related to the respect and elevation of women's voices. In fact, the data suggest that a university president who values women leaders does not inevitably correspond to the necessary backing required to secure essential supports and attrition points at varying apertures on a women's career journey to higher education leadership. In several cases, women expressed concerns at the faculty and department chair levels within academic units; they simultaneously cited praise for the university's leadership while identifying problematic structures and leaders who perpetuate gender-based discrimination and harassment at the college and unit levels.

The participants' stories reveal that they are paying attention: they notice when the President appoints women to positions across campus, they note the success of the prior MU Provost who went on to serve as the first woman president at another land-grant institution, and they appreciate that the number of women in leadership roles at MU is seemingly growing. And yet, the participants have also paid attention to the gender-biased ways that current and former women Provosts and Vice Presidents have been disrespected and continue to be discredited on campus every day. They acknowledged that the power structures that determine tenure and promotion are occurring at the men-dominated college levels. They hinted at the gender-biased hiring and recruiting processes which yield few, if any, woman candidates. And perhaps most prominently, they illuminated the notion that while they appreciate the President's leadership and the number of women represented near the top, there is still room for growth in ensuring that the women working in every university level and unit are not "left to our own devices."

The participant narratives, combined with the literature, raise the following question: if more women represented in leadership positions are necessary to eliminate gender-based discrimination, and men are continuously blocking women from advancing to leadership 
positions as evidenced by these narratives, how can the cycle be interrupted? Further, if there is an embedded fear of retaliation and negative consequence for reporting harassment and genderbased discrimination, and if those reporting lines are through men as gatekeepers, how can women safely report unfair treatment in the workplace in hopes of dismantling a system of ongoing subjugation?

The impact of representation and visibility for women in leadership positions was also clear throughout the participants' narratives; the women repeatedly mentioned how they observe and model behavior based on former and current women Provosts and Vice Presidents at MU. In doing so, they observe the ways in which men colleagues react to their leadership and they also observe tactics of leadership that they wish to emulate, all of which illuminates the need for more women to be represented at the highest levels of the university's leadership. A lack of women in top positions can reinforce entrenched beliefs that support leadership by only men and perpetuate a culture of workplace gender bias that can interrupt and hinder the leadership identity development of women within the organization (Ibarra et al., 2013). By acknowledging this underrepresentation, MU and similar universities can build cultures that advance the leadership identity development of women throughout multiple levels of the organization itself.

Michelle Obama (2020) recently affirmed the importance of diversity represented in leadership positions, specifically related to the empathy and understanding she received from a woman supervisor and mentor she had as a young professional. She explained, "Women bring a different perspective to the workplace that is important and relevant," (Obama, 2020, 00:14:05). Obama continued by explaining that her supervisor was innately aware of the challenges associated with being a woman and therefore, in tune with how to best support her in ways that perhaps a man might not directly relate to; she said, “...we need that feminine energy, that perspective, that approach," (Obama, 2020, 00:15:11). The findings from this study coincide 
with Obama's testimony; the call for more women to be represented as supervisors and in leadership positions is clear, especially in the environment of higher education. However, the narratives reveal further questions related to an ongoing cycle: if women leaders in higher education are unable to be their authentic selves in the workplace, employing strategies and tactics to navigate gender bias at every turn, do they have time and energy left over to devote to supervision and mentorship at the level necessary to cultivate and prepare young women to fill their shoes one day?

The importance of having women in key university leadership positions to learn from and model is evident, and as the participants expressed, the experience is unique to gender identity and how groups of individuals at the university receive and respect women in positions of power. As the findings revealed, many women are keenly watching the current Provost and Vice President for Academic Affairs, just as they had watched the former Provost and Vice President for Academic Affairs before her. If the women in high-level leadership roles at MU are all looking to the Provost, who does the Provost look to?

While this study was based on the experiences of women who currently work for MidAtlantic University, their narratives revealed findings that can be enacted at similar land-grant institutions or large, public research institutions in an analogous setting, making the results highly transferable. In fact, the study revealed no finding so specific that would distinguish the patterns of one land-grant institution from another similar large public or research university. For more than half of the participants in this study, there was no expressive connection between their work and MU's mission as a land-grant institution when asked directly; therefore, the researcher was unable to specifically explore certain aspects of every participants' identity through only the lens of being a woman leader at a land-grant institution, as had been initially intended. Though some participants mentioned the land-grant mission in connection to their commitment to serve 
the state, the concepts were all related to the nature of their work as opposed to their unique experiences as women administrators.

Further, while the study focused on telling the stories of women whose career paths led them to leadership positions at a land-grant institution and whose current working environment exists in a land-grant institution, their broad experiences and their multi-decade narratives spanned several institution and organization types, which reach beyond the scope of land-grant universities. Thus, most participants believed that their work and experience as a woman would be comparable at any large, public, or similar institution, especially when discussing their motivation to positively impact the lives of students and when offering advice to aspiring women leaders. In addition, with the majority of participants having worked outside of the institution and with many having experience outside of higher education in general, there are even greater opportunities for transferability when recognizing that many of the concepts referenced in their narratives occurred before their journey led them to Mid-Atlantic University. With this in mind, the findings of this study, while specific to one public, land-grant research institution, are transferable to similar organizations, colleges, universities, and contexts related to the broad experiences of women in leadership positions.

\section{Limitations and Context}

Although this study offers expedient insight into the career paths and ongoing leadership of women administrators at Mid-Atlantic University, the study was exploratory in nature and therefore, limited by the number of participants. To include the voices of 18 women in this study and their career development across several decades, the researcher had to limit parameters regarding how deeply each story was explored, analyzed, and shared. A similar study with a smaller number of participants could allow for longer, more focused interviews and a deeper analysis of each woman's reflections, at the risk of including fewer voices. 
While narrative inquiry through qualitative interviews was a suitable method of data collection for this study, it must be recognized that qualitative research methods, such as interviews, are not designed to epitomize hard facts. This study could gain credibility in the future by pairing the qualitative interviews with a quantitative survey and adequate analysis to strengthen the data behind specific patterns that were brought to light through the participants' stories, including additional demographic data.

Maintaining the confidentiality of the participants and their stories to mitigate the risk of identification, retaliation, or retribution also contributed to the limitation of this study; participant data was shared in segments, linked to a pseudonym when it was safe to do so. For many participants, their title, department name, or age, would immediately reveal their identities as they are either the only woman who met the study's recruitment criteria in that unit, the youngest woman in that unit, the only person with that specific title, or any combination of those factors.

It must be evidently acknowledged that in maintaining a clear focus on the women's narratives in this study, the researcher intentionally did not ask any direct questions about marriage, partners, or spousal support, nor any questions about the choices or experiences of maintaining a family while progressing through an advanced career trajectory. This decision was upheld with several priorities in mind, first and foremost, to offer respect to each individual participant in acknowledging her attainment. Additionally, it was important for the researcher to ensure that she was upholding an appropriate application of feminist theory by preventing the perpetuation of gender-based discrimination. This type of hidden stereotyping, so often embedded in interview questions, silently implies that women are unable to have both a career and a complete family while asserting that women must maintain a disproportionate amount of caregiving and service at home; it was central to the ethical foundation of this study to ensure that research participants were not asked to answer this type of question that is so infrequently 
asked of their men counterparts. The researcher also believed strongly that even if she had asked directly about the impact of a partner, family, and caregiving on one's career journey in this study, the findings would not be contributing any new or groundbreaking research to the already existing body of literature on the topic of work life balance for women in the workplace.

Thoughtfully considering the perspective of racial identities, this study lacked diversity primarily due to the lack of women whose titles qualified them for consideration in the sample. Though the researcher cannot account for the number of non-white women who hold the titles that qualify them for this study, additional research must be conducted to include other levels of responsibility to incorporate more voices. In addition, participants were not asked to self-identify beyond gender identity and age; therefore, the diversity of ability, sexual orientation, veteran status, socioeconomic status, and other qualifiers cannot be fully considered as a demographic reference in this study. Naturally, expanding this study's sample to include a broader range of titles and responsibilities along career paths for women in higher education will add organic diversity of identities and enrich the findings by amplifying the voices of more individuals. From a similar lens, it must be acknowledged that there were vital voices excluded from this study when the choice was made to target a sample of women whose career titles comprised the university's top leadership. Though this choice was intentional, focused on understanding the unique supports and barriers relative to their career advancement, there are still many more stories to tell of the women who did not encounter the same level of attrition points or experience the same propensity of support necessary to reach the highest level of a university's leadership; the stories left untold can be just as valuable.

It is also pertinent to address the global health crisis, political climate, racial unrest, unprecedented challenges for higher education, and uncharacteristic uncertainty associated with the timeframe of this study's data collection, which occurred in June 2020. While at the time of 
the research, there was only speculation and educated predictions regarding the potential impact of the pandemic on women, women caregivers, and women in educational careers; however, the anticipated drop in college enrollment and expected decrease in state appropriations to public institutions was front of mind for higher education leaders as they considered the challenges facing their staff related to budgeting, safety, and the potential for personnel elimination or furloughs. These factors, while not necessarily a traditional limitation of research, impacted the format of the data collection process as well as the recruitment of participants and, perhaps most prominently, the mindset of the participants while completing the research interviews.

\section{Coronavirus Pandemic}

“COVID-19 is not just altering everyday life; it's also upending psychological research," (Clay, 2020). As the world still faces COVID-19 and its bearing on life, industry, productivity, safety, family, wellness, and all aspects of the human experience, there is no research yet to identify how this pandemic impacts the process of qualitative research. However, what can be identified now, even at a premature stage, is that the fear, uncertainty, and change associated with it will continue to impact the lives of people everywhere, and for the purpose of this study, especially university administrators.

In March 2020, the World Health Organization determined the global outbreak of COVID-19 to be a pandemic spreading with severity at alarming rates (World Health Organization, 2020). While the impact of COVID-19 is still unfolding, there are immediate factors affecting the work, lives, and wellbeing of the participants in this study. Prior to and during spring break, Mid-Atlantic University made several immediate changes to mitigate risk related to the spread of COVID-19. Among these changes, announced in March 2020, was an extended spring break with a discouraged physical return to campus, a transition to online 
instruction for the second half of the Spring 2020 semester, and an immediate suspension on all university international and nonessential travel.

To support and enact these changes effectively and efficiently, the university's leadership and its departments faced a timely and unforeseen challenge ahead. As the participants in this study agreed to be interviewed in June 2020, their personal lives and workload had shifted to meet the ongoing challenges associated with a campus-wide effort to respond to COVID-19. Suddenly, university administrators and their teams were forced into unexpected conversations and protocols regarding immediate budget freezes, pending staff furlough, pauses in research activity, transition to remote work, adaptation to new technology, and in any situation, how to support one's team of students, faculty, or staff, as they face these challenges, while doing so in a virtual and remote working environment. It would be incongruous not to acknowledge the burden of leadership and associated ambiguity during this time, and its associated potential for impact on the participants' capacity to fully offer their narratives to the study amidst this multifaceted crisis.

While the interviews were intended to take place in person, COVID-19 and the stay-athome and social-distancing orders, as well as the university's virtual working environment recommendation, prevented in-person interviews and therefore, all participants were interviewed through the secure Zoom video platform. This sudden substitution of video calls for traditionally in-person or telephone meetings has potential to cause what experts identify as "zoom fatigue" (Petriglieri, as cited in Jiang, 2020). Being on video calls requires more focus than a face-to-face meeting, and for many participants who lead units and departments at the university, transition to remote work involved and continues to involve multiple video-conference meetings per day (Jiang, 2020). Though participants expressed their gratitude during the interview process to be “discussing something other than COVID-19", many hinted at the overall exhaustion of video 
conference meetings. In addition, the shift to remote work and adjustment of many traditional caregiving patterns, some participants were facing new caregiving responsibilities at home during the workday including but not limited to, caring for children and other family members simultaneously.

\section{Racial Unrest in America}

While countries, communities, universities, and families adapted their lives and work as a result of COVID-19, the United States experienced a reignition of the Black Lives Matter movement, which gained momentum after a series of incidents in which Black individuals were killed by law enforcement officers. During this time, Americans used their voices through inperson peaceful protests, online and virtual education, and social media activism to maintain a focus of national and international attention on the notion that Black lives should matter in America.

As America continues to grapple with both COVID-19 and racial injustice, the effects on its people, and the participants of this study related to their work, lives, family, wellness and stability, are yet to be determined and still unfolding every day. In relation to this unknown and pressing impact, Katti (2020) expressed, "We must recognize that our Black (and minority POC) students and colleagues are living in a reality very different from that experienced by the predominant groups in academia." He explained that black colleagues and students are living in a different reality than those of their white counterparts; attempting to establish normalcy during abnormal and racially charged moments in history such as these can be considered uncharted waters (Katti, 2020). To provide auxiliary perspective, Katti (2020, paragraph 5) said:

None of your Black students and colleagues have been able to sleep much, or focus on anything work-related, ever since Ahmaud Arbery was murdered while jogging, Breonna Taylor was shot eight times and killed in her bed by policemen barging into her home on 
a no-knock warrant and George Floyd had his breath choked out of his dying body as a policeman knelt on his neck while staring into a camera. The viral video shows that George Floyd expressed his inability to breathe multiple times during the eight minutes and 46 seconds, and that he eventually called out to his dead mother for help as a last resort. This was the video that sparked an uproar from people and brought everyone out to protest in the streets with an energy unlike anything we've seen in decades. Protests occurred all over the globe as the viral video reached people near and far. Even though COVID-19 is the present health scare, billions of people found it more important to express their disgust and disappointment with the treatment of POC.

In mid-June 2020, hundreds of people identified as “MU’s Concerned Black

Community" called for "swift, progressive change" in relation to diversity and inclusion on MidAtlantic University's campus. The administration responded by activating four campus-wide working groups to identify key action areas and recommend a plan for progress. This campuswide effort and the launch of working groups added a new and immediate call to action for many administrators at the university, including several participants in this study. With racial justice on campus reestablished as an immediate priority and with the uncertainty of the ongoing impact of COVID-19 on the university's instruction, finance, and overall function, the timeframe for participants was fraught with additional pressure, responsibility, and ambiguity.

\section{Directions for Future Research}

From a broad perspective, this study supports existing literature related to the eminence of women in society, the status of women in the workplace, and specifically, the unique lived experiences of women in higher education leadership working environments. Primarily, this study acknowledges the deficit in the number women who hold high-level leadership positions at universities and as the literature indicated, confirms that women's paths to higher education 
leadership are wrought with unique challenges, with barriers accumulating over time (Allan, 2011; ACE 2016; Ellemers, 2014).

This study supports and extends existing research related to formal and informal mentorship programs for women leaders in higher education; the importance of mentoring relationships and mentorship programs is clear (Brown, 2005; Dunbar \& Kinnersley, 2011; Jackson et al., 2017). The subset of the data related to supervisory relationships, recruitment processes, and advocacy by mentors contributes to the research as well. Men, who continue to hold the majority of leadership positions in higher education, maintain access to the gatekeeping processes which can prevent or promote women's advancement. The participant's narratives confirmed the presence of gender rules and unfair expectations of women in the higher education workplace, which validates and extends the prior research; this culture, which favors masculinebased norms and obstructs opportunities for women to advance, is still permeating in today's university environment. Despite the current body of research on the topic, combined with the new narratives contributed from this study, the prevalence of these barriers and challenges for women identifies an immediate need for further research in a broad context and more deeply within individual institutions and units.

Existing research confirmed that higher education relegates women and continues to perpetuate cumulative oppression across generations (Pasque, 2014). Prior research also indicated that women bear an unreasonably large share of the service burden in the higher education setting (Guarino \& Borden, 2017; Heilman et al., 2004; O’Meara et al., 2017; Pasque, 2014); participants substantiated this pattern. Participants in the study who identified as women of color shared their acknowledgement of the heavier service burden for minority women and women in marginalized and underrepresented groups. Participants also validated the calls for a more equitable representation of women in higher education leadership and simultaneously 
identified a growing need for investigation into the gaps across intersecting identities and the influences that impede or accelerate the path (Allan, 2011; Pasque, 2014). While this study, rooted in feminist and role congruity theory, explores these gaps, the necessity for deeper acknowledgement and thorough synthesis of rich narratives remains apposite.

Researchers have evaluated the leadership effectiveness of men and women and its impact on organizations, but there has been no direct application to the impact of majority men leadership at land-grant institutions or its relation to hurdles early in the pipeline for women to be represented in leadership roles (Clark, 2006; Ibarra et al., 2013). Though evidence indicates that the pipeline for leaders in higher education is preparing more qualified women than men, there are consistently fewer women than men in high-level faculty and leadership positions (Johnson, 2016). Because many of the participants followed what they deemed to be an indirect path to their current roles, they were not expecting this type of career outcome at the beginning of their careers; therefore, they were not able to reasonably reflect on opportunities they might have gained or missed related to higher education leadership as young adults. A multi-year, longitudinal study of women with career higher education leadership aspirations would offer insightful research that addresses gaps related to barriers and attrition points as early in the pipeline as enrollment in graduate school. By studying their experiences over an extended period, the participants' choices, support systems, aspirations, and philosophy could be tracked, leading to the identification of specific strategies and cultural influences that will open doors for women administrators in the future. To extend even those findings, a parallel study could be conducted with a sample of men participants.

Several opportunities for future research related to the intersection of woman's identities could add depth and additional findings to this study, including a collection and analysis of targeted demographic information. As evidenced by the participants' remarks regarding the 
cultural tax, service burden, and nuanced expectations of women of color, there are many patterns and experiences to be further explored. Additionally, there is no major research to identify the influence of this men-dominant leadership structure and how the future of higher education and a land-grant institution like Mid-Atlantic University could be impacted with a more gender-representative leadership team. Likewise, the research is minimal related to the experiences of "only women" on a team or within a department, and the unique barriers and opportunities that coincide with that experience. As women break into more leadership roles in higher education, there will be growing instances of their presence as "the only woman" or "the only woman of color" or "the only woman and only caregiver", each wrought with lived experiences that will differ from those of the men who are accustomed to leading in similar environments.

There is a limited body of research related to modern experiences, environments, and campus culture at land-grant institutions. This study was initially designed to contribute data and relevant findings specific to the culture of a land-grant institution; however, the participants' narratives revealed that their personal and professional experiences are not closely tied with the land-grant mission. In addition, many of the participants admitted unfamiliarity with the landgrant mission itself and confirmed that their narratives regarding women's leadership and career paths were not specifically tied to an institution type. With this in mind, there is an ongoing need for further research and exploration into various facets of campus culture and the experiences of women leaders who are more closely affiliated with the land-grant mission and purpose.

\section{Conclusion}

Prior to this study, there was no significant research related to career paths for aspiring women leaders at a land-grant university in reference to the barriers, culture, and tactics employed to access and succeed in high-level leadership positions within this institutional 
setting. The findings have shed light on specific barriers, attrition points, and tactics, but leave ample opportunity and further questions related to eliminating, overcoming, and changing the culture for leaders in the future. If there are tactics, as the findings identified, that women employ daily to attain and maintain their positions among the university's leadership, what are the tactics that their men counterparts can activate in order to support the women's success and job security?

Given these findings and the questions that remain, there is a requisite for deeper and more analytic research on the current landscape for aspiring and surviving women leaders, as early as enrollment in graduate school, to continue to identify the barriers and supports within these systems that influence women's ascension to senior leadership positions. While this study has contributed new and diverse voices to the existing literature, which has tended to refer to women as a monolithic group over time, future research will allow for heartening insights into the lived experiences of distinct and aspiring leaders who identify as women.

While we focus on the narratives of women to illuminate the inequities in this system, it must be noted that this is not, in fact, a women's issue at all; the barriers for women in higher education raise questions of objectivity and place detrimental limitations on the success of educational institutions overall (West \& Curtis, 2006). If land-grant institutions like Mid-Atlantic University are not consistently prioritizing the advancement of women to attain and maintain higher education leadership positions, the pervasiveness of gender-based discrimination will continue to override the university's culture, hindering opportunity for growth and advancement. Without necessary changes to organizational culture and norms, colleges and universities could miss out on the quality talent and potential of women leaders, and others, who might not perfectly fit the formerly and assiduously defined model of success based on the default of men's leadership (Eddy \& Ward, 2015). 
The results of this study suggested that there are structures in place that must be prioritized and adapted to continue advancing opportunities for women to lead in the landscape of higher education. Among these existing initiatives is a recurring emphasis on strong supervisory relationships, opportunities for positive role-modeling and extensive representation, avenues to cultivate vast and supportive networks, and dedicated time, resources, and support for formal and informal mentoring relationships. In addition, there are cultural aspects of the higher education leadership working environment at Mid-Atlantic University and other institutions that must be immediately addressed related to gender-based discrimination, inherent bias, sexual harassment, patriarchal culture, gender-typing, unequal service burdens, and the silencing and devaluing of women in the workplace of higher education leadership.

While there has been progress to note over decades of advancement and steps to mitigate the underrepresentation of women specifically, it is clear that there is a significant amount of work to be done at Mid-Atlantic University and beyond. This underrepresentation has resulted in a waste of talent for women; while women possess the potential to be transformative leaders in higher education institutions, there is no time more critical than right now to re-socialize the system and prime women leaders to help meet the current challenges of the industry (Dunn et al., 2014). Hopefully, the requisite to authorize and extend this research will diminish over time as the instance of women in higher education leadership roles becomes less rare and more universally accepted, as women are increasingly represented at levels of university leadership. Until then, we know one thing for sure: the women who served as participants in this study have committed to creating brave spaces for those who will come after them. If they continue to accomplish at the rate they have already persisted, there will be clearer pathways ahead. 


\section{References}

Acker, J. (1990). Hierarchies, jobs, and bodies: A theory of gendered organizations. Gender \& Society, 4(2), 139-158. https://doi.org/10.1177/089124390004002002

Acker, J. (2006). Inequality regimes: Gender, class, and race in organizations. Gender \& Society, 20(4), 441-464. https://doi.org/10.1177/0891243206289499

Airini, S., Conner, L., McPherson, K., Midson, B., \& Wilson, C. (2011). Learning to be leaders in higher education: What helps or hinders women's advancement as leaders in universities. Educational Management Administration \& Leadership, 39(1), 44-62. https://doi.org/10.1177/1741143210383896

Allan, E. J. (2011). Women's status in higher education: Equity matters. ASHE Higher Education Report, 37(1), iii-163. https://doi.org/10.1002/aehe.3701

Allan, E. J. (2014). Multiple perspectives for creating change in the academy. In K. De Welde, A. Stepnick, \& P.A. Pasque (Eds.), Disrupting the culture of silence: Confronting gender inequality and making change in higher education (pp. 293-302). Stylus Publishing.

Allen, K. R., \& Jaramillo-Sierra, A. L. (2015). Feminist theory and research on family relationships: Pluralism and complexity. Sex Roles, 73(3-4), 93-99. https://doi.org/10.1007/s11199-015-0527-4

American Association of University Professors [AAUP] (2011). It's not over yet: The annual report on economic state of the profession, 2010-11. http://www.aaup.org/reportspublications/2010-11salarysurvey

Association of Public and Land-Grant Universities [APLU] (2019). What is a land-grant university? https://www.aplu.org/about-us/history-of-aplu/what-is-a-land-grant$\underline{\text { university/ }}$ 
Ballakrishnen, S., Fielding-Singh, P., \& Magliozzi, D. (2019). Intentional invisibility: Professional women and the navigation of workplace constraints. Sociological Perspectives, 62(1), 23-41. https://doi.org/10.1177/0731121418782185

Ballenger, J. (2010). Women's access to higher education leadership: Cultural and structural barriers. Forum on Public Policy, 2010(5). https://forumonpublicpolicy.com/vol2010no5/womencareers2010.html

Bell, E., Meriläinen, S., Taylor, S., \& Tienari, J. (2019). Time's up! Feminist theory and activism meets organization studies. Human Relations, 72(1), 4-22. https://doi.org/10.1177/0018726718790067

Bianco, M. (2016, April 30). Academia is quietly and systematically keeping its women from succeeding. Quartz. https://qz.com/670647/academia-is-quietly-and-systematicallykeeping-its-women-from-succeeding/

Bilen-Green, C., Froelich, K. A., \& Jacobson, S. W. (2008). The prevalence of women in academic leadership positions, and potential impact on prevalence of women in the professorial ranks. WEPAN conference proceedings, 1-11. https://www.ndsu.edu/fileadmin/forward/documents/WEPAN2.pdf

Bird, S. R. (2011). Unsettling universities' incongruous, gendered bureaucratic structures: A case-study approach. Gender, Work \& Organization, 18(2), 202-230. https://doi.org/10.1111/j.1468-0432.2009.00510.x

Born, A., Ranehill, E., \& Sandberg, A. (2018). A man's world? The impact of a male dominated environment on female leadership. SSRN Electronic Journal. https://doi.org/10.2139/ssrn.3207198 
Borzelleca, D. (2012, February 16). The male-female ratio in college. Forbes. https://www.forbes.com/sites/ccap/2012/02/16/the-male-female-ratio-incollege/\#7b1f4c2cfa52

Brieger, S. A., Francoeur, C., Welzel, C., \& Ben-Amar, W. (2019). Empowering women: The role of emancipative forces in board gender diversity. Journal of Business Ethics, 155(2), 495-511. https://doi.org/10.1007/s10551-017-3489-3

Broido, E. M., Brown, K. R., Stygles, K. N., \& Bronkema, R. H. (2015). Responding to gendered dynamics: Experiences of women working over 25 years at one university. The Journal of Higher Education, 86(4), 595-627. https://doi.org/10.1353/jhe.2015.0023

Brown, T. M. (2005). Mentorship and the female college president. Sex Roles, 52(9), 659-666. https://doi.org/10.1007/s11199-005-3733-7

Bruckmüller, S., \& Branscombe, N.R., (2010). The glass cliff: When and why women are selected as leaders in crisis contexts. British Journal of Social Psychology, 49, 433-451. https://doi.org/10.1348/014466609X466594

Bruckmüller, S., Ryan, M. R., Haslam, S. A., \& Peters, K. (2013). Ceilings, cliffs, and labyrinths: Exploring metaphors for workplace gender discrimination. In M. K. Ryan \& N. R. Branscombe (Eds.), The SAGE handbook of gender and psychology (pp. 450-464). SAGE. https://doi.org/10.4135/9781446269930.n27

Campbell, M. L. (1998). Institutional ethnography and experience as data. Qualitative Sociology 21(55). https://doi.org/10.1023/A:1022171325924

Chafetz, J. S. (2004). Bridging feminist theory and research methodology. Journal of Family Issues, 25(7), 963-977. https://doi.org/10.1177/0192513X04267098 
Chafetz, J. S. (2004). Gendered power and privilege: Taking Lenski one step further. Sociological Theory, 22(2), 269-277. https://doi.org/10.1111/j.0735$\underline{2751.2004 .00217 . x}$

Chilwniak, L. (1997). Higher education leadership: Analyzing the gender gap. ASHE-ERIC Higher Education Report, 25(4). The George Washington Graduate School of Education and Human Development. https://files.eric.ed.gov/fulltext/ED410847.pdf

Cho, A., Chakraborty, D., \& Rowland, D. (2017). Gender representation in faculty and leadership at land-grant and research institutions. Agronomy Journal, 109(1), 14. https://doi.org/10.2134/agronj2015.0566

Clance, P. R., \& Imes, S. A. (1978). The imposter phenomenon in high achieving women: Dynamics and therapeutic intervention. Psychotherapy: Theory, Research and Practice, 15(3), 241-247. https://doi.org/10.1037/h0086006

Clark, H. (2006, March 8). Are women happy under the glass ceiling? Forbes. https://www.forbes.com/2006/03/07/glass-ceiling-opportunities-cx_hc_0308glass.html\#6be2db5d3e39

Clay, R. A. (2020, March 19). Conducting research during the COVID-19 pandemic. http://www.apa.org/news/apa/2020/03/conducting-research-covid-19

Collins, J. (2011). Good to great. Harper Business. https://doi.org/10.1002/hrdq.1092

Cook, S. G. (2012), Women presidents: Now $26.4 \%$ but still underrepresented. Women in Higher Education, 21 (5), 1-3. https://doi.org/10.1002/whe.10322

Davis, S., \& Brown, K. (2017). Automatically discounted: Using black feminist theory to critically analyze the experiences of black female faculty. NCPEA International Journal of Educational Leadership Preparation, 12(1). https://files.eric.ed.gov/fulltext/EJ1145466.pdf 
Davis, T. J., \& Cooper, D. L. (2017). “People are messy”: Complex narratives of supervising new professionals in student affairs. Journal of Student Affairs Research and Practice, 54(1), 55-68. https://doi.org/10.1080/19496591.2016.1219267

Denzin, N. K. (2009). The elephant in the living room: Or extending the conversation about the politics of evidence. Qualitative Research, 9(2), 139-160. https://doi.org/10.1177/1468794108098034

Derks, B., Van Laar, C., \& Ellemers, N. (2016). The queen bee phenomenon: Why women leaders distance themselves from junior women. The Leadership Quarterly, 27(3), 456469. https://doi.org/10.1016/j.leaqua.2015.12.007

DeVault, M., \& Gross, G. (2012). Feminist qualitative interviewing: Experience, talk, and knowledge. In S. Hesse-Biber (Ed.), Handbook of feminist research: Theory and praxis (pp. 206-236). SAGE. https://doi.org/10.4135/9781483384740

DeVault, M., and McCoy, L. (2002) Institutional ethnography: Using interviews to investigate ruling relations. In J. Gubrium \& J. Holstein (Eds.), Handbook of interview research: Context \& method (pp. 751-776). SAGE. https://doi.org/10.4135/9781412973588.d43

De Welde, K., \& Stepnick, A. (2015). Introduction. In K. De Welde, A. Stepnick, \& P.A. Pasque (Eds.), Disrupting the culture of silence: Confronting gender inequality and making change in higher education (1-26). Stylus Publishing.

Dominici, F., Fried, L. P., and Zeger, S. L. (2009). So few women leaders. Academe, 95(4), 2527. https://www.aaup.org/article/so-few-women-leaders\#.Xi-GwhNKjq0

Doucet, A., \& Mauthner, N. (2012). Knowing responsibly: Ethics, feminist epistemologies and methodologies. In T. Miller, M. Birch, M. Mauthner, \& J. Jessop (Eds.), Ethics in qualitative research (pp. 122-139). SAGE. https://doi.org/10.4135/9781473913912 
Dunbar, D., and Kinnersley, R.T. (2011). Mentoring female administrators toward leadership success. The Delta Kappa Gamma Bulletin, 77(3). Delta Kappa Gamma Society International. http://www.deltakappagamma.org/NH/DKGbulletinspring2011.PDF

Dunn, D., Gerlach, J. M., and Hyle, A. E. (2014). Gender and leadership: Reflections of women in higher education administration. International Journal of Leadership and Change, 2(1). http://digitalcommons.wku.edu/ijlc/vol2/iss 1/2

Eagly, A. H. (2007). Female leadership advantage and disadvantage: Resolving the contradictions. Psychology of Women Quarterly, 31(1), 1-12. https://doi.org/10.1111/j.1471-6402.2007.00326.x

Eagly, A. H., \& Carli, L. L. (2007). Through the labyrinth: The truth about how women become leaders. Harvard Business School Press.

Eagly, A. H., \& Karau, S. J. (2002). Role congruity theory of prejudice toward female leaders. Psychological Review, 109(3), 573-598. https://doi.org/10.1037//0033-295X.109.3.573

Eckel, P.D. (2019). An international visitor's guide to understanding university governing boards in the United States of America. Alliance for Higher Education and Democracy (Penn AHEAD), University of Pennsylvania Graduate School of Education. https://repository.upenn.edu/ahead_papers/5/

Eddy, P., \& Ward, K. (2015) Lean in or opt out: Career pathways of academic women, change: The Magazine of Higher Learning, 47(2), 6-13.

\section{https://doi.org/10.1080/00091383.2015.1018082}

El-Alayli, A., Hansen-Brown, A.A. \& Ceynar, M. (2018). Dancing backwards in high heels: Female professors experience more work demands and special favor requests, particularly from academically entitled students. Sex Roles, 79, 136-150. https://doi.org/10.1007/s11199-017-0872-6 
Ellemers, N. (2014). Women at work: How organizational features impact career development. Policy Insights from the Behavioral and Brain Sciences, 1(1), 46-54. https://doi.org/10.1177/2372732214549327

Ely, R. J., and Meyerson, D. E. (2000). Advancing gender equity in organizations: The challenge and importance of maintaining a gender narrative. Organization 7, 589-608. https://doi.org/10.1177/135050840074005

Fain, P. (2010, November 29). Diversity remains fleeting on colleges' governing boards, surveys find. The Chronicle of Higher Education. https://www.chronicle.com/article/Diversity$\underline{\text { Remains-Fleeting-on/125566 }}$

Flaherty, C. (2018, January 10). Dancing backwards in high heels. Inside Higher Ed. https://www.insidehighered.com/news/2018/01/10/study-finds-female-professorsexperience-more-work-demands-and-special-favor

Fochtman, M. M. (2011). High achieving academic women, balancing multiple roles: How do they do it? In P. A. Pasque \& S. E. Nicholson (Eds.), Empowering women in higher education and student affairs: Theory, research, narrative and practice from feminist perspectives (pp. 85-103). Stylus and the American College Personnel Association.

Ford, L.E. (2016) Two steps forward, one step back? Strengthening the foundations of women's leadership in higher education. Politics, Groups, and Identities, 4(3), 499-512, https://doi.org/10.1080/21565503.2016.1170705

Frances, C. (2018). The status of women in American higher education. Sociology and Anthropology, 6(9), 695-708. https://doi.org/10.13189/sa.2018.060902

Funk, K. (2019). If the shoe fits: Gender role congruity and evaluations of public managers. Journal of Behavioral Public Administration, 2(1). https://doi.org/10.30636/jbpa.21.48 
Gagliardi, J. S., Espinosa, L. L., Turk, J. M., \& Taylor, M. (2017) American college president study 2017. American Council on Education. https://www.tiaainstitute.org/publication/american-college-president-study-2017

Gangone, L. M., \& Lennon, T. (2014) Benchmarking women leadership in academia and beyond. In K. Longman \& S. Madsen (Eds.), Women and leadership in higher education (pp. 3-22). Information Age Publishing.

Gardiner, R.A. (2014). Women leaders, authenticity, and higher education: Convictions and contradictions. In K. Longman \& S. Madsen (Eds.), Women and leadership in higher education (pp. 153-168). Information Age Publishing.

Gavazzi, S. M., Gee, E.G., \& Magrath, C.P. (2018). Land-grant universities for the future. Johns Hopkins University Press. https://doi.org/10.1353/book.62441

Gipson, A. N., Pfaff, D. L., Mendelsohn, D. B., Catenacci, L. T., \& Burke, W. W. (2017). Women and leadership: Selection, development, leadership style, and performance. The Journal of Applied Behavioral Science, 53(1), 32-65. https://doi.org/10.1177/0021886316687247

Glover, M. H. (2012). Existing pathways: A historical overview of black women in higher education administration. In T. Jones, J. Brazzell, M. McClinton, \& M. Glover (Eds.), Pathways to higher education administration for African American women. Stylus Publishing.

Guarino, C. (2017). Why higher ed needs to get rid of the gender gap for "academic housekeeping." The Conversation. http://theconversation.com/why-higher-ed-needs-toget-rid-of-the-gender-gap-for-academic-housekeeping-82135 
Guarino, C. M., \& Borden, V. M. H. (2017). Faculty service loads and gender: Are women taking care of the academic family? Research in Higher Education, 58(6), 672-694. https://doi.org/10.1007/s11162-017-9454-2

Guillaume, R. O., Martinez, E., \& Elue, C. (2019). Social media use, legitimacy, and imposter phenomenon: A collaborative autoethnography among early career faculty. Journal of Ethnographic \& Qualitative Research, 14(2), 125-136.

Gumpertz, M., Durodoye, R., Griffith, E., \& Wilson, A. (2017). Retention and promotion of women and underrepresented minority faculty in science and engineering at four large land-grant institutions. PLOS ONE, 12(11), e0187285. https://doi.org/10.1371/journal.pone.0187285

Harley, D. A. (2008). Maids of academe: African American women faculty at predominantly white institutions. Journal of African American Studies, 12, 19-36.

\section{https://doi.org/10.1007/s12111-007-9030-5}

Hart, A. W. (2014). Docs, jocks, and other wildlife. In Longman, K., \& Madsen, S. (Eds.) Women and leadership in higher education (pp. 197-204). Information Age Publishing.

Heilman, M. E., Wallen, A. S., Fuchs, D., \& Tamkins, M. M. (2004). Penalties for success: Reactions to women who succeed at male gender-typed tasks. Journal of Applied Psychology, 89(3), 416-427. https://doi.org/10.1037/0021-9010.89.3.416

Henneberry, S. R., Valdivia, C., \& Wells, B.L. (2002). Women in higher education: Social sciences at land grant universities in the U.S. Working Papers 92909, University of Missouri Columbia, Department of Agricultural Economics. https://doi.org/10.22004/ag.econ.92909

Hesse-Biber, S., \& Leavy, P. (2007). Feminist research practice: A primer. SAGE. 
Hill, L. H., \& Wheat, C. A. (2017). The influence of mentorship and role models on university women leaders' career paths to university presidency. The Qualitative Report, 22(8), 2090-2111. https://nsuworks.nova.edu/tqr/vol22/iss8/2/

Hironimus-Wendt, R.J., \& Dedjoe, D.A. (2015) Glass ceilings and gated communities in higher education. In K. De Welde, A. Stepnick, \& P.A. Pasque (Eds.), Disrupting the culture of silence: Confronting gender inequality and making change in higher education (pp. 3754). Stylus Publishing.

Hirshfield, L., \& Joseph, T.D. (2011). 'We need a woman, we need a black woman': gender, race, and identity taxation in the academy. Gender and Education, 24(2), 213-227. https://doi.org/10.1080/09540253.2011.606208

Howard, E., \& Gagliardi, J. (2017). Leading the way to parity: Preparation, persistence, and the role of women presidents. American Council on Education. https://www.acenet.edu/Documents/Leading-the-Way-to-Parity.pdf

Huffstutler, S. Y., \& Varnell, G. (2006). The imposter phenomenon in the new nurse practitioner graduates. Advanced Practice Nursing Journal, 6(2). https://www.medscape.com/viewarticle/533648

Ibarra, H., Ely, R., \& Kolb, D. (2013) Women rising: The unseen barriers. Harvard Business Review, 91(101). https://doi.org/10.2469/dig.v43.n5.1

Jackson, J. K., Latimer, M., \& Stoiko, R. (2017). The dynamic between knowledge production and faculty evaluation: Perceptions of the promotion and tenure process across disciplines. Innovative Higher Education, 42(3), 193-205. https://doi.org/10.1007/s10755-016-9378-3

Jacobson, J. (2002, March 5). Moving up from student affairs, or staying put. The Chronicle of Higher Education. https://www.chronicle.com/article/Moving-Up-from-Student/45970 
Jalalzai, F. (2008). Women rule: Shattering the executive glass ceiling. Politics \& Gender, 4(02). https://doi.org/10.1017/S1743923X08000317

Jiang, M. (2020, April 22). The reason Zoom calls drain your energy. British Broadcasting Company. https://www.bbc.com/worklife/article/20200421-why-zoom-video-chats-areso-exhausting

Johnson, H. L. (2016.) Pipelines, pathways, and institutional leadership: An update on the status of women in higher education. Washington, DC: American Council on Education. https://www.acenet.edu/Documents/Higher-Ed-Spotlight-Pipelines-Pathways-and$\underline{\text { Institutional-Leadership-Status-of-Women.pdf }}$

Kaplan, S., \& Tinsley, A. (1989). The unfinished agenda: Women in administration of higher education. Education Digest, 55(4), 24 -27. https://eric.ed.gov/?id=EJ385702

Kark, R., \& Eagly, A.H. (2010). Gender and leadership: Negotiating the labyrinth. In J.C. Chrisler \& D.R. McCreary (Eds.), Handbook of gender research in psychology. https://doi.org/10.1007/978-1-4419-1467-5_19

Karkouti, M. (2016). Professional leadership practices and diversity issues in the U.S. higher education systems: A research synthesis. Education, 136(4), 405-412. https://eric.ed.gov/?id=EJ1104195

Katti, M. (2020, July 8). COVID-19 and the black lives matter movement: Managing academic realities. American Society for Microbiology. https://asm.org/Articles/2020/July/COVID19-and-the-Black-Lives-Matter-Movement-Manag

Kellerman, B. (2003). You've come a long way, baby - and you've got miles to go. In D. L. Rhode (Ed.), The difference “difference” makes: Women and leadership (pp. 3-53). Stanford University Press. 
Kellerman, B., \& Rhode, D. L. (2014) Women at the top: The pipeline reconsidered. In K. Longman \& S. Madsen (Eds.), Women and leadership in higher education (pp. 23-41). Information Age Publishing.

Kellerman, B., \& Rhode, D. L. (2017). Women at the top: The pipeline as pipe dream. About Campus, 21(6), 11-18. https://doi.org/10.1002/abc.21275

Keohane, N. O., (2014) Leadership out front and behind the scenes: Young women's ambitions for leadership today. In K. Longman \& S. Madsen (Eds.), Women and leadership in higher education (pp. 41-59). Information Age Publishing.

Kerr, C., \& Gade, M. (1989). The guardians: Boards of trustees of American colleges and universities. Association of Governing Boards.

Kezar, A. (2014). Women's contribution to leadership and the road ahead. In K. Longman \& S. Madsen (Eds.), Women and leadership in higher education (pp. 117-134). Information Age Publishing.

Khalid, A. (2016) Women and leadership in higher education. Journal of Workplace Learning, 28(1), pp. 49-50, https://doi.org/10.1108/JWL-09-2015-0071

Khwaja, T., Eddy, P. L., \& Ward, K. (Eds.), (2017). Critical Approaches to Women and Gender in Higher Education: Reaching the tipping point for change. https://doi.org/10.1057/978$\underline{1-137-59285-9}$

Kohli, W., \& Burbules, N. (2012). Feminisms and educational research. Rowman \& Littlefield. Komter, A. (1991). Gender, power and feminist theory. In K. Davis, M. Leijenaar, \& J. Oldersma (Eds.), The gender of power (pp. 42-62). SAGE.

Kowalski-Braun, M. (2014). An examination of how feminist perspectives and generational differences influence the leadership practices of women administrators in higher education. Dissertations. 381. http://scholarworks.wmich.edu/dissertations/381 
Krefting, L. (1991). Rigor in qualitative research: The assessment of trustworthiness. American Journal of Occupational Therapy, 45(3), 214-222. https://doi.org/10.5014/ajot.45.3.214

Letherby, G. (2003). Feminist research in theory and practice. Open University Press.

Lichtman, M. (2014). Qualitative research for the social sciences. SAGE. https://doi.org/10.4135/9781544307756

Lincoln, Y., \& Guba, E. (1985). Naturalist Inquiry. SAGE.

Lombardo, G. (2017). The social sciences and ethical protocols and standards for research in social sciences today. In R. Iphofen (Ed.), Finding common ground - Consensus in research ethics across the social sciences: consensus in research ethics across the social sciences (pp. 177-184). Emerald Publishing.

Longman, K.A., \& Madsen, S. (2014). Women and leadership in higher education. Information Age Publishing.

Maguire, P., \& Berge, B. (2009). Elbows out, arms linked: Claiming spaces for feminisms and gender equity in educational action research. In S. E. Noffke \& B. Somekh (Eds.), The SAGE handbook of educational action research (pp. 399-408). SAGE. https://doi.org/10.4135/9780857021021.n37

Mainah, F., \& Perkins, V. (2015). Challenges facing female leaders of color in U.S. higher education. International journal of African Development, 2(2).

Mandel (2003). A question about women and the leadership option. In D. L. Rhode (Ed.), The difference "difference” makes: Women and leadership (pp. 59-66). Stanford University Press.

Marshall, C., Johnson, M., \& Edwards, T. (2017). A feminist critical policy analysis of patriarchy in leadership. In M. D. Young \& S. Diem (Eds.), Critical Approaches to 
Education Policy Analysis (Vol. 4, pp. 131-150). https://doi.org/10.1007/978-3-319$\underline{39643-9 \quad 7}$

Marshall, S. M., Gardner, M. M., Hughes, C., \& Lowery, U. (2016). Attrition from student affairs: Perspectives from those who exited the profession. Journal of Student Affairs Research and Practice, 53(2), 146-159. https://doi.org/10.1080/19496591.2016.1147359

McCall, L. (2005). The complexity of intersectionality. Signs: Journal of Women in Culture and Society, 30(3), 1771-1800. https://doi.org/10.1086/426800

McCluney, C.L., \& Rabelo, V.C., (2019). Conditions of visibility: An intersectional examination of Black women's belongingness and distinctiveness at work. Journal of Vocational Behavior, 113, 143-152. https://doi.org/10.1016/j.jvb.2018.09.008

McGinn, M. K. (2012). Credibility. In A. Mills, G. Durepos \& E. Wiebe (Eds.) Encyclopedia of Case Study Research. https://doi.org/10.4135/9781412957397

McKinsey \& Company (2019). Women in the workplace. LeanIN.org. https://wiwreport.s3.amazonaws.com/Women_in the Workplace_2019_print.pdf

Mohr, T. S. (2014, August 25) Why women don't apply for jobs unless they're $100 \%$ qualified. Harvard Business Review. https://hbr.org/2014/08/why-women-dont-apply-for-jobsunless-theyre-100-qualified

Naples, N. (2003). Feminism and method: Ethnography, discourse analysis, and activist research. Routledge.

National Center for the State Courts [NCSC] (2019). Gender and racial fairness resource guide. https://www.ncsc.org/Topics/Access-and-Fairness/Gender-and-Racial-Fairness/Resource$\underline{\text { Guide.aspx }}$

National Research Council [NRC] (2005) Advancing Scientific Research in Education. Committee on Scientific Principles for Education Research. L. Towne, L. Wise and T.M. 
Winters (Eds.). Center for Education, Division of Behavioral and Social Sciences. The National Academies Press.

Nebraska Sociological Feminist Collective. (1988). A feminist ethic for social science research. E. Mellen Press.

Niskodé-Dossett, A. S., Pasque, P.A., \& Nicholson, S. E., (2011). Envisioning a new future with feminist voices: Research and practice from feminist perspectives. In P. A. Pasque \& S. E. Nicholson (Eds.), Empowering women in higher education and student affairs:

Theory, research, narrative and practice from feminist perspectives (pp. 325-333). Stylus and the American College Personnel Association.

O’Meara, K., Kuvaeva, A., Nyunt, G., Waugaman, C., \& Jackson, R. (2017). Asked more often: Gender differences in faculty workload in research universities and the work interactions that shape them. American Educational Research Journal, 54(6), 1154-1186. https://doi.org/10.3102/0002831217716767

Obama, M. (Host). (2020, September 9). Working women: Valerie Jarrett and the importance of mentorship (No. 7) [Audio podcast episode]. In The Michelle Obama Podcast. Higher Ground. https://open.spotify.com/episode/1DOjQtP8k7tdQybs7mYK49

Oikelome, G. (2017). Pathway to the presidency: the perceived impact of identity structures on the journey experiences of women college presidents. International Journal of Multicultural Education, 19(3), 19. https://doi.org/10.18251/ijme.v19i3.1377

Padilla, A. M. (1994). Ethnic minority scholars, research, and mentoring: Current and future issues. Educational Researcher, 23(4), 24-7. https://doi.org/10.3102/0013189X023004024

Parker, P. (2015). The historical role of women in higher education. Administrative Issues Journal Education Practice and Research, 5(1). https://doi.org/10.5929/2015.5.1.1 
Parkman, A. (2016). The imposter phenomenon in higher education: Incidence and impact. Journal of Higher Education Theory and Practice, 16(1), 51-60. http://www.nabusinesspress.com/JHETP/ParkmanA_Web16_1_.pdf

Pasque, P. (2014). Access and equity for women in higher education during the era of the Obama administration: Social identities, inequities, and the power of women's groups in changing leadership paradigms. In E. Zamani-Gallaher (Ed.), The Obama Administration and Educational Reform. Emerald Publishing.

Pasquerella, L., \& Clauss-Ehlers, C. S. (2017). Glass cliffs, queen bees, and the snow-woman effect: Persistent barriers to women's leadership in the academy. Liberal Education, Spring, (pp. 8-13). https://www.aacu.org/liberaleducation/2017/spring/pasquerella_clauss-ehlers

Paustian-Underdahl, S. C., Walker, L. S., \& Woehr, D. J. (2014). Gender and perceptions of leadership effectiveness: A meta-analysis of contextual moderators. Journal of Applied Psychology, 99(6), 1129-1145. https://doi.org/10.1037/a0036751

Peterson, H. (2014). An academic 'glass cliff'? Exploring the increase of women in Swedish higher education management. Athens Journal of Education 1(1), 33-44. https://doi.org/10.30958/aje.1-1-3

Peterson, H. (2016). Is managing academics “women's work”? Exploring the glass cliff in higher education management. Educational Management Administration \& Leadership 1(16). https://doi.org/10.1177/1741143214563897

Preissle, J. \& Han, Y. (2012). Feminist research ethics. In S. Hesse-Biber, S. (Ed.), Handbook of feminist research: Theory and praxis (pp. 583-605). SAGE. https://doi.org/10.4135/9781483384740 
Pullen, A., and Rhodes, C. (2015) Writing, the feminine and organization. Gender, Work and Organization 22(2), 87-93. https://doi.org/10.1111/gwao.12084

Pushor, D., \& Clandinin, D. (2009). The interconnections between narrative inquiry and action research. In S. E. Noffke \& B. Somekh (Eds.), The SAGE handbook of educational action research (pp. 290-300). SAGE. https://doi.org/10.4135/9780857021021.n27

Pyke, K. (2015). Faculty gender inequity and the "just say no to service" fairy tale. In K. De Welde, A. Stepnick \& P.A. Pasque (Eds.), Disrupting the culture of silence: Confronting gender inequality and making change in higher education (pp. 83-95). Stylus Publishing.

Rabovsky, T., \& Lee, H. (2018). Exploring the antecedents of the gender pay gap in U.S. higher education: Exploring the antecedents of the gender pay gap in U.S. higher education. Public Administration Review, 78(3), 375-385. https://doi.org/10.1111/puar.12827

Reis, T. C. (2015). Leadership stories: Defining gender in university leadership. Journal of Women in Educational Leadership, 70. http://digitalcommons.unl.edu/jwel/70

Reis, T. C., \& Grady, M. L. (2018). Women as university presidents: Navigating the administrative labyrinth. Leadership and Research in Education, 4. https://eric.ed.gov/?id=EJ1174445

Ridgeway, C., and S. Correll. (2004). Unpacking the gender system: A theoretical perspective on gender beliefs and social expectations. Gender \& Society, 18(4), 510-31. https://doi.org/10.1177/0891243204265269

Ritter, B. A., \& Yoder, J. D. (2004). Gender differences in leader emergence persist even for dominant women: An updated confirmation of role congruity theory. Psychology of Women Quarterly, 28(3), 187-193. https://doi.org/10.1111/j.1471-6402.2004.00135.x

Rosener, J. B. (1990, November 1). Ways Women Lead. Harvard Business Review. https://hbr.org/1990/11/ways-women-lead 
Roulston, K. (2014). Analyzing interviews. In U. Flick (Ed.) The SAGE handbook of qualitative data analysis (297-312). SAGE. https://doi.org/10.4135/9781446282243

Ryan, M. K., \& Haslam, S. A. (2005). The glass cliff: Evidence that women are over-represented in precarious leadership positions. British Journal of Management, 16, 81-90. https://doi.org/10.1111/j.1467-8551.2005.00433.x

Ryan, M. K., \& Haslam, S. A. (2007). The glass cliff: Exploring the dynamics surrounding the appointment of women to precarious leadership positions. Academy of Management Review, 32(2), 549-579. https://doi.org/10.5465/AMR.2007.24351856

Sabharwal, M. (2015). From glass ceiling to glass cliff: Women in senior executive service. Journal of Public Administration Research and Theory. https://doi.org/10.1093/jopart/mut030

Saunders, B., Kitzinger, J., \& Kitzinger, C. (2015). Anonymising interview data: challenges and compromise in practice. Qualitative Research, 15(5), 616-632. https://doi.org/10.1177/1468794114550439

Schwartz, M. P. (2010). How diverse are governing boards? How diverse should they be? Trusteeship, 18(6). https://agb.org/trusteeship-article/how-diverse-are-governingboards-how-diverse-should-they-be/

Searby, L., Ballenger, J., \& Tripses, J. (2015). Climbing the ladder, holding the ladder: The mentoring experiences of higher education female leaders. Advancing Women in Leadership, 35, 98-107. https://pdfs.semanticscholar.org/fd07/7d3a457507e4836c556da21197fa1a6394ae.pdf?.g $\underline{\mathrm{a}=2.239672454 .465509355 .1605664139-1068760882.1605664139}$

Smith, D. (2005). Institutional ethnography: A sociology for people. AltaMira Press. 
Sorber, N. (2013). Introduction. In R. Geiger \& N. Sorber (Eds.), The land-grant colleges and the reshaping of American higher education. (2013). Transaction.

Sulpizio, L. (2014) Developing women's leadership: An innovative and unique approach to raising leadership capacity. In K. Longman \& S. Madsen (Eds.), Women and leadership in higher education. Information Age Publishing.

Taylor, S. J., Bogdan, R., \& DeVault, M. (2015). Introduction to qualitative research methods: A guidebook and resource. John Wiley \& Sons.

Teague, L. W. J., \& Bobby, K. (2014). ACE's IDEALS for women leaders: Identify, develop, encourage, link, and support. In K. Longman \& S. Madsen (Eds.), Women and leadership in higher education (pp. 59-76). Information Age Publishing.

The White House Project. (2009). The white house project: Benchmarking women's leadership. The White House Project. http://www.in.gov/icw/files/benchmark_wom_leadership.pdf

Thorne, A. C. (1985). Visible and invisible women in land-grant colleges, 1890-1940. Faculty Honor Lectures. Paper 2. https://digitalcommons.usu.edu/honor_lectures/2

Trahan, M., \& Growe, R. (2012) Inequalities of women: Understanding marginalization of female educators and the quest for change. Focus on Colleges, Universities, and Schools, $6(1), 2-12$. http://www.nationalforum.com/Electronic\%20Journal\%20Volumes/Trahan, \%20Mitzi\%2 0Inequalities $\% 20$ of $\% 20$ Women $\% 20$ FOCUS $\% 20$ V6\%20N1\%202012.pdf

Turner, C. S. V., González, J. C., \& Wong, K. (2011). Faculty women of color: The critical nexus of race and gender. Journal of Diversity in Higher Education, 4(2), 199-211. https://doi.org/10.1037/a0024630 
Tuttle, K. N. (2004). The historical perspective of women administrators in higher education. Paper presented at the 2004 NASPA Alice Manicur Symposium. http://www.naspa.org/prodev/regforms/he04.pdf

United Nations Development Programme (2020). Tackling social norms: A game changer for gender inequalities. 2020 Human Development Perspectives. http://hdr.undp.org/sites/default/files/hd_perspectives_gsni.pdf

West, M. S., \& Curtis, J.W. (2006). AAUP Faculty Gender Equity Indicators 2006. American Association of University Professors. https://www.aaup.org/reportspublications/publications/see-all/aaup-faculty-gender-equity-indicators-2006

Wolf, M. (1992) A thrice told tale: Feminism, postmodernism and ethnographic responsibility. Stanford University Press.

Wolfinger, N.H. (2013). For female scientists, there's no good time to have children. The Atlantic. https://www.theatlantic.com/sexes/archive/2013/07/for-female-scientists-theresno-good-time-to-have-children/278165/

World Health Organization (2020). Archived WHO Timelines - COVID-19. https://www.who.int/news-room/detail/27-04-2020-who-timeline---covid19?gclid=Cj0KCQjwpNr4BRDYARIsAADIx9yIzZN70K1UUaiKuUiKuzt2c81x6OuTXf96-ZZYbjKtIDfIX3nuTwaAuLoEALw_wcB

Wyatt, M., \& Silvester, J. (2015). Reflections on the labyrinth: Investigating black and minority ethnic leaders' career experiences. Human relations, 68(8), 1243-1269. $\underline{\text { https://doi.org/10.1177/0018726714550890 }}$

Yakaboski, T., \& Donahoo, S. (2011). In (re)search of women in student affairs administration. In P. A. Pasque \& S. Errington Nicholson (Eds.), Empowering women in higher 
education and student affairs: Theory, research, narrative and practice from feminist perspectives (pp. 270-286). Stylus and the American College Personnel Association.

Young, M. (2003). Considering (irreconcilable?) Contradictions. In M. Young \& L. Skrla (Eds.), Reconsidering feminist research in educational leadership. State University of New York Press.

Yousafzai, M. [United Nations Youth Assembly] (2013, July 12). Malala Yousafzai addresses United Nations Youth Assembly [Video].

https://www.youtube.com/watch?v=3rNhZu3ttIU\&feature $=\mathrm{emb}$ title\&ab_channel=Unite $\underline{\text { dNations }}$ 


\section{Appendix A: Recruitment Email}

Date: June 2, 2020

Subject: Request for Interview

Dear [participant],

I'm writing to request your participation in an interview centered around the experiences of women leaders in higher education. As a $\mathrm{PhD}$ candidate, my dissertation research study aims to identify the barriers and attrition points for women administrators at a land-grant institution in hopes of forging clear paths for women in the future.

Participation will involve a 60-minute interview conversation, completed by telephone or virtually through Zoom. The attached letter references the parameters of your participation in the study. By agreeing to participate, it is implied that you have read the letter, understand your rights, and consent to contribute to this research.

I recognize that your time is incredibly valuable. If you are able to spare an hour for this interview, you'd be contributing knowledge to further the advancement of women in higher education.

If you are willing to participate in this study, please confirm by Friday, June $5^{\text {th }}$. After your confirmation, I will be in touch to schedule an interview time that is most convenient for you.

For more information about this study, please contact me through the information listed below. Thank you in advance for your consideration.

Sincerely, Meridith

Meridith Balas

PhD Candidate, Higher Education

West Virginia University

Meridith.Balas@mail.wvu.edu

724-984-8965 (cell)

Study Title: Examining the Career Pathways for Women Administrators at a Land-Grant University 


\section{Appendix B: IRB Cover Letter}

\section{West VirginiaUniversity $r$ cover Letter
OfFICE OF HUMAN RESEARCH PROTECTION}

Dear Participant,

This letter is a request for you to take part in a research project aiming to understand the lived experiences of women in leadership positions at land-grant institutions. This project is being conducted by Meridith Balas, a $\mathrm{PhD}$ Candidate within the Department of Curriculum and Instruction/Literacy Studies at WVU under the supervision of Dr. Erin McHenry-Sorber, Assistant Professor and Program Coordinator in the Department of Curriculum and Instruction/Literacy Studies, to fulfill the requirements for a Doctorate of Philosophy in Higher Education.

If you decide to participate, you will be asked to partake in an interview over the phone or through the Zoom video platform. Your participation in this project will take approximately 60-90 minutes, with an opportunity to extend the time or schedule a follow-up conversation if you wish to do so. You must be 18 years of age or older to participate. You will not receive compensation for participating in this study.

Your involvement in this project will be kept as confidential as legally possible. Data will be reported in the aggregate. The interviews will be audio-recorded for the researcher's references; all files will be saved on a secure, password-protected server separately from the list of participant names. Your participation is completely voluntary. You may skip any question that you do not wish to answer and you may discontinue at any time. West Virginia University's Institutional Review Board approval of this project is on file.

If you have any questions about this research project, please feel free to contact me at 724-984-8965 or by e-mail at Meridith.Balas@mail.wvu.edu; Dr. Erin McHenry-Sorber can be reached via email at ECMchenrysorber@mail.wvu.edu. If you have any questions about your rights as a research participant, please contact the WVU Office of Human Research Protection by phone at 304-293-7073 or by email at $\underline{\text { IRB@mail.wvu.edu. }}$

I hope that you will participate in this research project, as it could help us better understand the barriers and attrition points for women pursuing and succeeding in leadership positions at land-grant universities. Thank you for your time and consideration.

Sincerely,

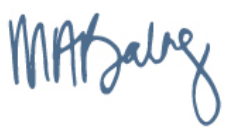

Meridith A. Balas

Meridith.Balas@mail.wvu.edu

724-984-8965

Phone: $304-293-7073$ Fax: 304-293-3098 http://oric.research.wvu.ed
Chestnut Ridge Research Building

886 Chestnut Ridge Road

PO Box 6845

Morgantown, WV 26506-6845
P a g e 1

V2019.06.03 


\section{Appendix C: Interview Guide}

Informant:

Title:

Date:

Time of Interview:

Introduction

1. PhD Candidate in Higher Education - this research will culminate in my doctoral dissertation examining the career pathways for academic and non-academic administrators at a land-grant institution.

2. Interview will be 45-60 minutes.

a. Primary goal: understand your story and your perspective, especially related to your career trajectory and the experiences you had and have as a woman leader at a land-grant institution.

3. You are under no obligation to answer any/all questions. You can skip any question and/or stop the interview at any time.

4. This will be audio recorded today for the researcher's reference.

5. Do you have any questions for me before we get started?

Background Information

- Could you start by telling me about yourself? (R3)

$\circ$ What is you educational/professional background?

- [if not addressed, when did they start at MU specifically? Were they at similar institutions before?]

- Could you tell me (a little bit more) about your current role and responsibilities? (R1)

$\circ$ What were the positive influences that helped you to attain this position?

- Could you tell me more about that?

- Mentorship?

- Specific skills/experience?

- Network?

- Behavior modeling?

$\circ$ Were there any barriers/challenges that you encountered in reaching this position? (R1; R2)

- Were any of these related to your identity as a woman?

- Could you tell me more about that?

- Childcare?

- Masculine culture?

- Discrimination?

- Pay?

- Service load?

Current Role / Environment / Future

- Have you considered seeking another leadership role from here? (R1; R2; R3)

If so, what attracts you about that role?

- What do you believe has helped to prepare you for this role?

$\circ$ Do you foresee any barriers? 
- Have you ever been discouraged from pursuing another leadership role? (R3)

If so, why?

- How would you describe the environment you work in, related to your identity as a woman? (R1; R2)

O (Dependent) are there tactics you employ to navigate this environment?

- There is evidence to suggest that women are burdened with significantly more servicerelated tasks in the higher education workplace, often having to put in more hours and offer more care for their colleagues and communities. Does this resonate with you? (R1; $\mathrm{R} 2$ )

- Could you tell me more about that?

- How did that make you feel?

- How did that impact your ability to do your job?

- Research also suggests that women faced gender-based discrimination at various levels, including implicit bias and a toxic patriarchal culture. Have you found or do you find this to be true? (R2)

- Could you tell me about a time when you might have felt that colleagues or leaders have had a bias against you because you are a woman? (R1)

- Could you tell me more about that?

- How did that make you feel?

- How did that impact your ability to do your job?

Land-Grant

- Do you find any aspects of your experience obtaining and maintaining your position to be unique to working for a land-grant institution? (R1; R2)

Future

- If you could offer advice to an aspiring woman administrator what would you share with them? (R1)

- What advice would you offer to man in that same capacity?

- You are considered a woman leader at MU. What does that mean to you? 